\title{
Palladium-Catalyzed Diastereoselective Formal [5+3] Cycloaddition for the Construction of Spirooxindoles Fused with an Eight-membered Ring
}

\author{
Ben Niu, ${ }^{a}$ Xiao-Yun Wu, ${ }^{a}$ Yin Wei, ${ }^{\mathrm{c}}$ and Min Shi*a,b,c
}

${ }^{a}$ Key Laboratory for Advanced Materials and Institute of Fine Chemicals, School of Chemistry \& Molecular Engineering, East China University of Science and Technology, Meilong Road No. 130, Shanghai, 200237 P. R. China.

${ }^{b}$ Shenzhen Grubbs Institute, Southern University of Science and Technology, Shenzhen, Guangdong 518000, China

'State Key Laboratory of Organometallic Chemistry, Center for Excellence in Molecular Synthesis, University of Chinese Academy of Sciences, Shanghai Institute of Organic Chemistry, Chinese Academy of Sciences, 345 Lingling Road, Shanghai 200032 P. R. China. E-mail: weiyin@sioc.ac.cn, mshi@mail.sioc.ac.cn

Content

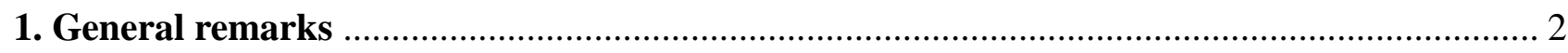

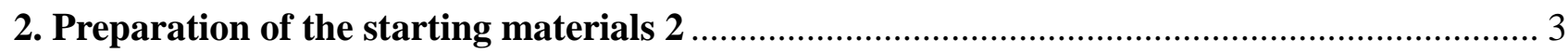

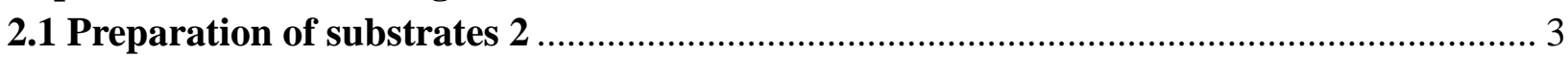

3. Preliminary attempts to synthesize 4 aa in a one-pot manner....................................... 4

Scheme S1 Preliminary attempts to synthesize 4aa in one-step............................................ 4

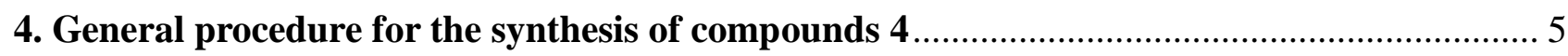

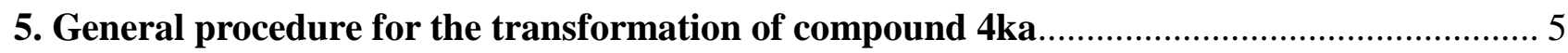

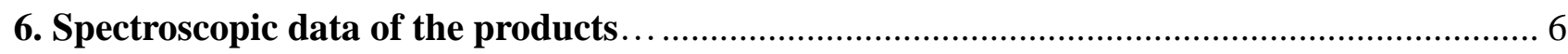

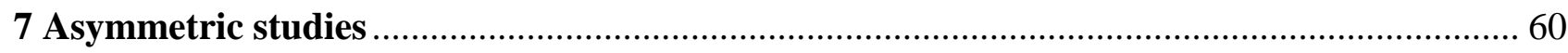

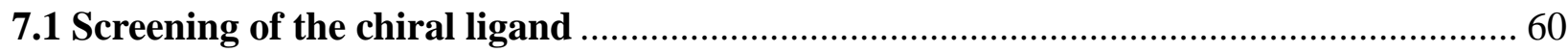

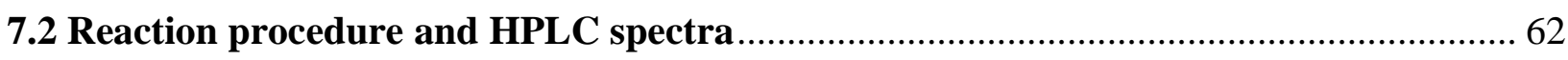

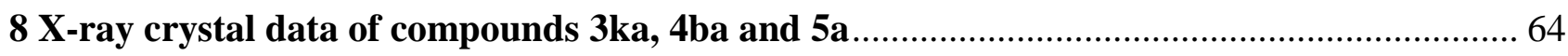




\section{General remarks}

Melting points were determined on a digital melting point apparatus and temperatures were uncorrected. ${ }^{1} \mathrm{H}$ NMR spectra were measured on a Brucker AC 400 or Agilent $(400 \mathrm{MHz})$ spectrometer. Data were reported as follows: chemical shifts in ppm referenced to the internal solvent signal (peak at $0.00 \mathrm{ppm}$ in the case of $\mathrm{CDCl}_{3}$ with tetramethylsilane as an internal standard), multiplicity ( $\mathrm{s}=$ singlet, $\mathrm{d}=$ doublet, $\mathrm{t}=$ triplet, $\mathrm{m}=$ multiplet $)$, coupling constants $(\mathrm{Hz})$, and assignment. ${ }^{13} \mathrm{C}$ NMR spectra were measured on a Brucker AC 400 (100 MHz) spectrometer with complete proton decoupling. Chemical shifts were reported in ppm from the internal solvent signal (peak at $77.000 \mathrm{ppm}$ in the case of $\mathrm{CDCl}_{3}$ ). Infrared spectra were recorded on a Perkin-Elmer PE-983 spectrometer with absorption in $\mathrm{cm}^{-1}$. The reference of ${ }^{19} \mathrm{~F}$ NMR (376 MHz) spectra is trichlorofluoromethane. Flash column chromatography was performed using 300-400 mesh silica gel. For thin-layer chromatography (TLC), silica gel plates (Huanghai GF254) were used. Chiral HPLC was performed on a SHIMADZU SPD-10A $v p$ series with chiral columns (Chiralpak IB, column $4.6 \times 250 \mathrm{~mm}$, (Daicel Chemical Ind., Ltd.)). Mass spectra were recorded by ESI, and HRMS was measured on a HP-5989 instrument. The employed solvents were dry up by standard methods when necessary. Commercially obtained reagents were used without further purification. 


\section{Preparation of the starting materials 2.}

\subsection{Preparation of substrates 2}

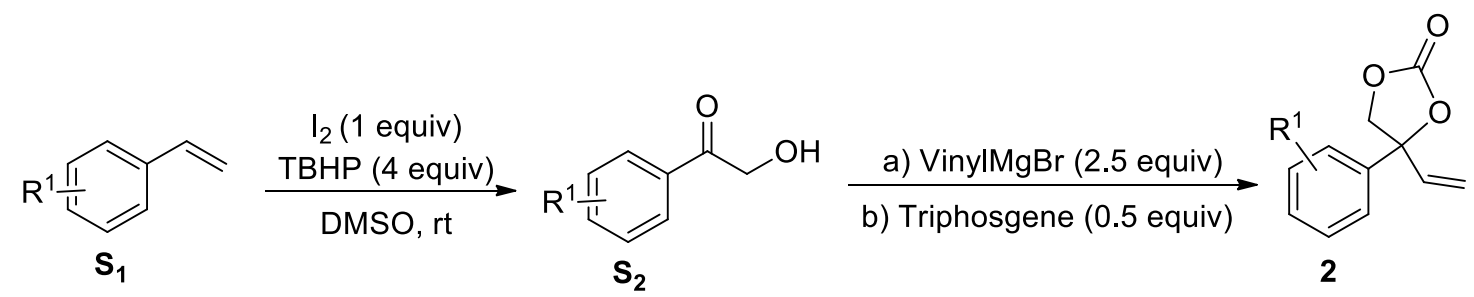

A mixture of styrene $\mathbf{S}_{\mathbf{1}}(20.0 \mathrm{mmol})$, iodine $(20.0 \mathrm{mmol}, 1.0$ equiv), and TBHP (4.0 mmol, 4.0 equiv, $65 \%$ aq. solution) in DMSO $(30.0 \mathrm{~mL})$ was stirred at room temperature till almost full conversion of the substrates by TLC analysis, and then extracted with EtOAc $(3 \times 30 \mathrm{~mL})$. The organic phase was washed with $10 \% \mathrm{Na}_{2} \mathrm{~S}_{2} \mathrm{O}_{3}$. After drying over $\mathrm{Na}_{2} \mathrm{SO}_{4}$ and evaporation under reduced pressure, the crude product was purified by a column chromatography on silica gel (eluent: petroleum ether/EtOAc) to afford the product $\mathbf{S}_{2}$.

To a solution of $\alpha$-hydroxyacetophenones $\mathbf{S}_{\mathbf{2}}$ (1.0 equiv) in THF was added slowly vinylmagnesium bromide (1.0 M in THF, 2.5 equiv) at $0{ }^{\circ} \mathrm{C}$ and the reaction mixture was stirred under $\mathrm{N}_{2}$ atmosphere at room temperature for $2 \mathrm{~h}$. The reaction was then quenched with saturated aqueous $\mathrm{NH}_{4} \mathrm{Cl}$ and extracted with EtOAc. The combined organic layers were dried over anhydrous $\mathrm{Na}_{2} \mathrm{SO}_{4}$, filtered and concentrated. The residue was purified by a flash chromatography on silica to afford corresponding diols.

Triphosgene ( 0.5 equiv) was dissolved in dry $\mathrm{CH}_{2} \mathrm{Cl}_{2}$ and was added to a solution of diol (1.0 equiv) and pyridine (4.0 equiv) in dry $\mathrm{CH}_{2} \mathrm{Cl}_{2}$ at $0{ }^{\circ} \mathrm{C}$. The reaction mixture was stirred under $\mathrm{N}_{2}$ atmosphere at room temperature for $2 \mathrm{~h}$. The reaction was then quenched with saturated aqueous $\mathrm{NH}_{4} \mathrm{Cl}$ and extracted with $\mathrm{CH}_{2} \mathrm{Cl}_{2}(3 \times 30 \mathrm{~mL})$. The combined organic layers were dried over anhydrous $\mathrm{Na}_{2} \mathrm{SO}_{4}$, filtered and concentrated. The residue was purified by a flash chromatography on silica to afford corresponding VECs 2. 
3. Preliminary attempts to synthesize 4aa in a one-pot manner.
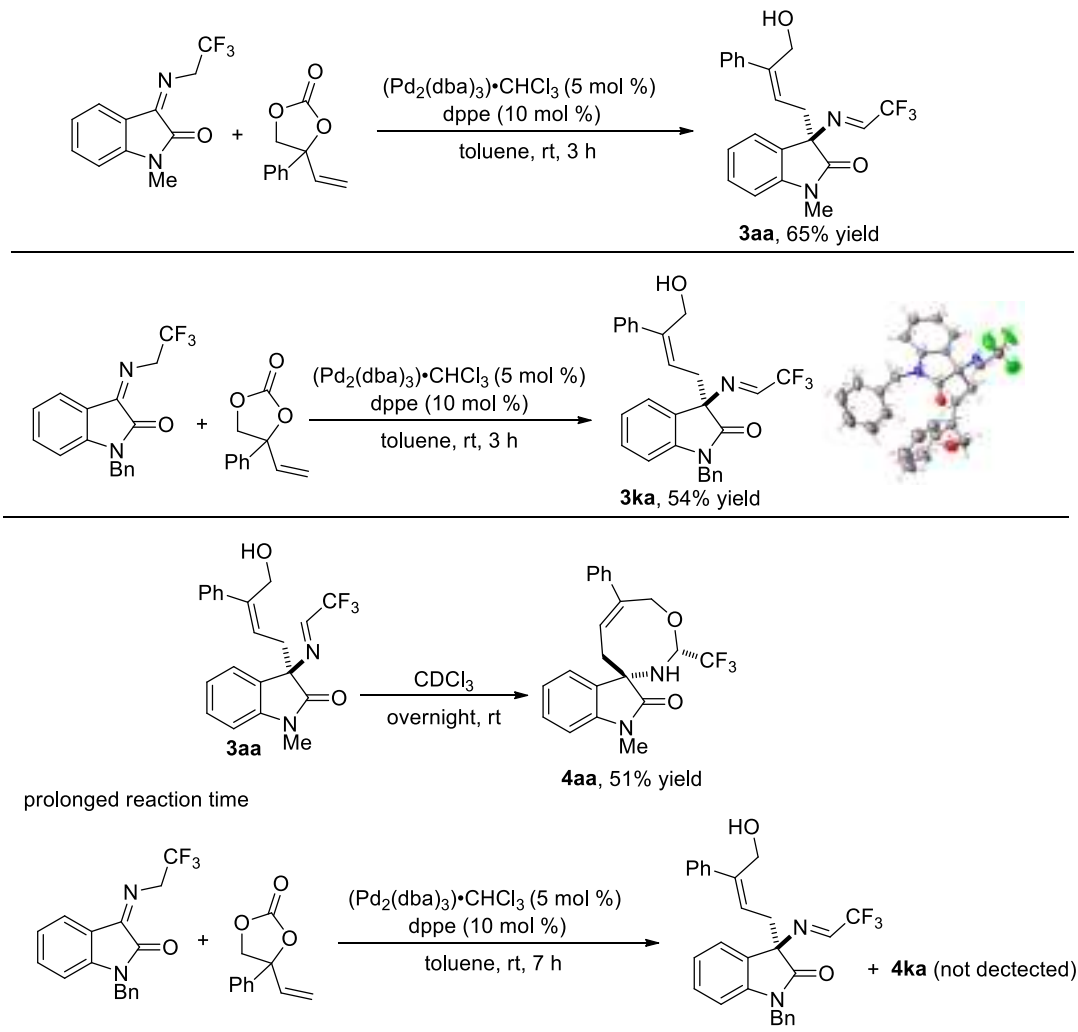

3ka, $61 \%$ yield

one-pot:<smiles>CN1C(=O)/C(=N\CC(F)(F)F)c2ccccc21</smiles><smiles>C=CC1(c2ccccc2)COC(=O)O1</smiles>

1) $\left(\mathrm{Pd}_{2}(\mathrm{dba})_{3}\right) \cdot \mathrm{CHCl}_{3}(5 \mathrm{~mol} \%)$ dppe $(10 \mathrm{~mol} \%)$

toluene, $\mathrm{rt}, 3 \mathrm{~h}$<smiles>CN1C(=O)[C@@]2(CC=C(c3ccccc3)CO[C@H](C(F)(F)F)N2)c2ccccc21</smiles>

4aa, $70 \%$ yield

2)<smiles>C=CC1(c2ccccc2)COC(=O)O1</smiles>

$\left(\mathrm{Pd}_{2}(\mathrm{dba})_{3}\right) \cdot \mathrm{CHCl}_{3}(5 \mathrm{~mol} \%)$

dppe $(10 \mathrm{~mol} \%)$

( \pm )-PA (20 mol \%)

toluene, $\mathrm{rt}, 3 \mathrm{~h}$

3)

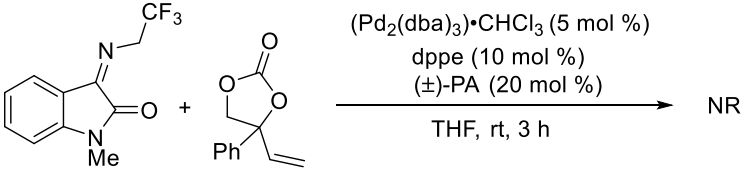

Scheme S1 Preliminary attempts to synthesize 4aa in one-step 
4. General procedure for the synthesis of compounds 4 .

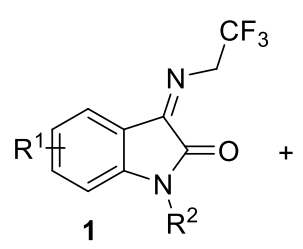

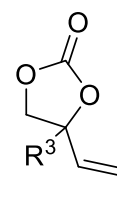

2

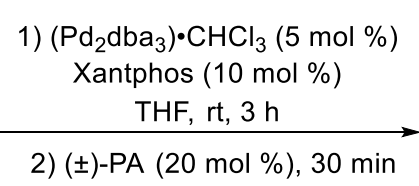

2) ( \pm )-PA (20 mol \%), $30 \mathrm{~min}$

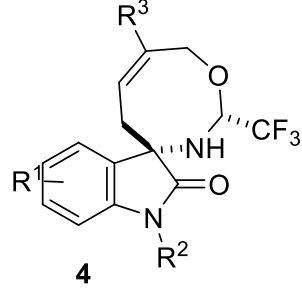

In an oven dried $10 \mathrm{~mL}$ Schlenk tube equipped with a magnetic stirring bar, Pd-catalyst $(0.05$ equiv) and Xantphos (0.1 equiv) was stirred in THF $(2.0 \mathrm{~mL})$ for $1.5 \mathrm{~h}$. After that, VEC $2(0.2$ mmol, 1.0 equiv), isatin-derived imide 1 ( $0.26 \mathrm{mmol}, 1.3$ equiv) were added under nitrogen atmosphere. The reaction mixture was stirred at room temperature till almost full conversion of the substrate 2 by TLC analysis. Then, ( \pm )-PA ( $0.04 \mathrm{mmol}, 0.2$ equiv) was added and the reaction mixture was continuously stirred for 30 minutes. The solvent was evaporated under vacuum and the residue was purified by a column chromatography (eluent: petroleum ether:ethyl acetate $=10: 1$ to $15: 1)$ to afford the desired product 4.

\section{General procedure for the transformation of compound $4 \mathrm{ka}$.}
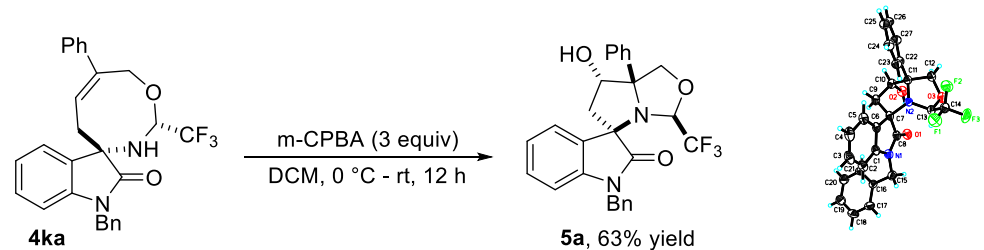

X-ray structure of $5 a$

In an oven dried $10 \mathrm{~mL}$ Schlenk tube equipped with a magnetic stirring bar, 4ka ( $0.5 \mathrm{mmol}, 1$ equiv) and m-CPBA (1.5 mmol, 3 equiv) were added under nitrogen atmosphere, and then, dichloromethane $(2 \mathrm{~mL})$ was added. The reaction mixture was stirred at $0^{\circ} \mathrm{C}$ for $12 \mathrm{~h}$. The solvent was evaporated under vacuum and the residue was purified by a column chromatography (eluent: petroleum ether:ethyl acetate $=10: 1$ to $15: 1)$ to afford the desired product 5a. 


\section{Spectroscopic data of the products.}<smiles>CN(C(=O)[C@H](N=CC(F)(F)F)c1ccccc1)c1ccccc1</smiles>

Compound 3aa: Yield: $50 \mathrm{mg}, 65 \%$; A yellow liquid; ${ }^{1} \mathrm{H}$ NMR (400 MHz, Chloroform-d) $\delta 7.92$ (d, $J=3.2 \mathrm{~Hz}, 1 \mathrm{H}), 7.48(\mathrm{~d}, J=7.2 \mathrm{~Hz}, 1 \mathrm{H}), 7.38(\mathrm{t}, J=7.6 \mathrm{~Hz}, 1 \mathrm{H}), 7.32-7.14(\mathrm{~m}, 6 \mathrm{H}), 6.91(\mathrm{~d}, J=$ $8.0 \mathrm{~Hz}, 1 \mathrm{H}), 5.41(\mathrm{dd}, J=9.6,6.8 \mathrm{~Hz}, 1 \mathrm{H}), 4.57(\mathrm{dd}, J=12.4,4.4 \mathrm{~Hz}, 1 \mathrm{H}), 4.31(\mathrm{dd}, J=12.4,7.6$ $\mathrm{Hz}, 1 \mathrm{H}), 3.20(\mathrm{~s}, 3 \mathrm{H}), 3.17-2.98(\mathrm{~m}, 2 \mathrm{H}), 2.40-2.25(\mathrm{~m}, 1 \mathrm{H}) ;{ }^{13} \mathrm{C} \mathrm{NMR}\left(100 \mathrm{MHz}, \mathrm{CDCl}_{3}\right) \delta$ $174.4,150.5$ (q, $J=38.7 \mathrm{~Hz}), 144.7,142.5,140.9,129.9,128.4,128.0,127.5,126.3,124.5,123.8$, $122.1,118.9(\mathrm{q}, J=273.9 \mathrm{~Hz}), 117.5,109.0,72.5,59.6,37.4,26.3 ;{ }^{19} \mathrm{~F} \mathrm{NMR}(376 \mathrm{MHz}$, Chloroform- $d$ ) $\delta-71.36\left(\mathrm{~d}, J=3.0 \mathrm{~Hz}\right.$ ); IR (neat): $v$ 3484, 2937, 1700 1488, 1170, 749, $695 \mathrm{~cm}^{-1}$; HRMS (ESI) Calcd. for $\mathrm{C}_{21} \mathrm{H}_{20} \mathrm{~F}_{3} \mathrm{~N}_{2} \mathrm{O}_{3}[\mathrm{M}+\mathrm{H}]^{+}:$389.1471, found: 389.1462 .

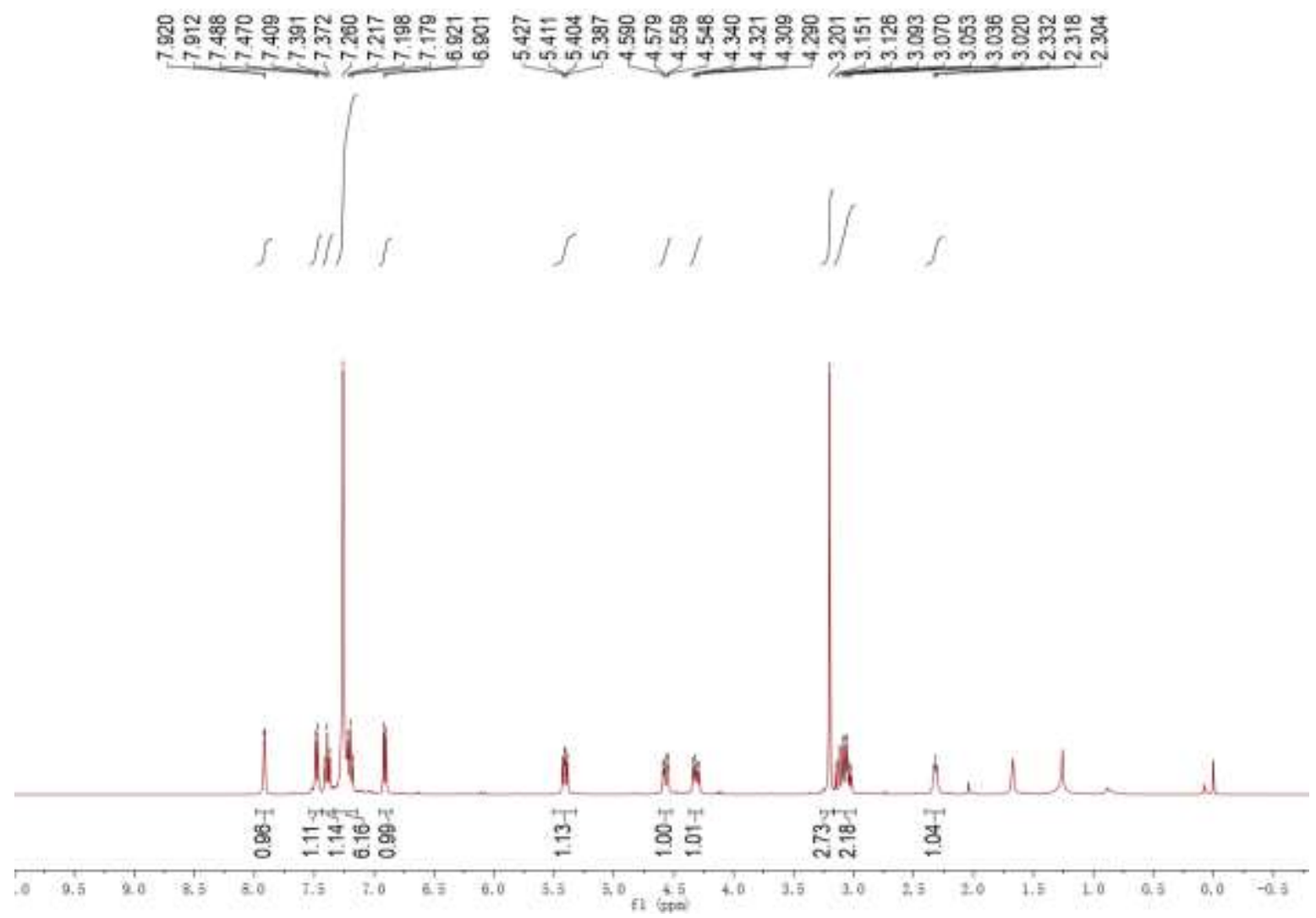




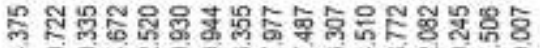

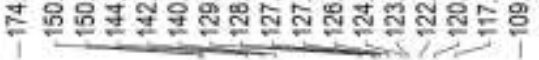

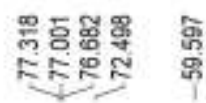

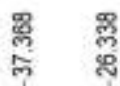
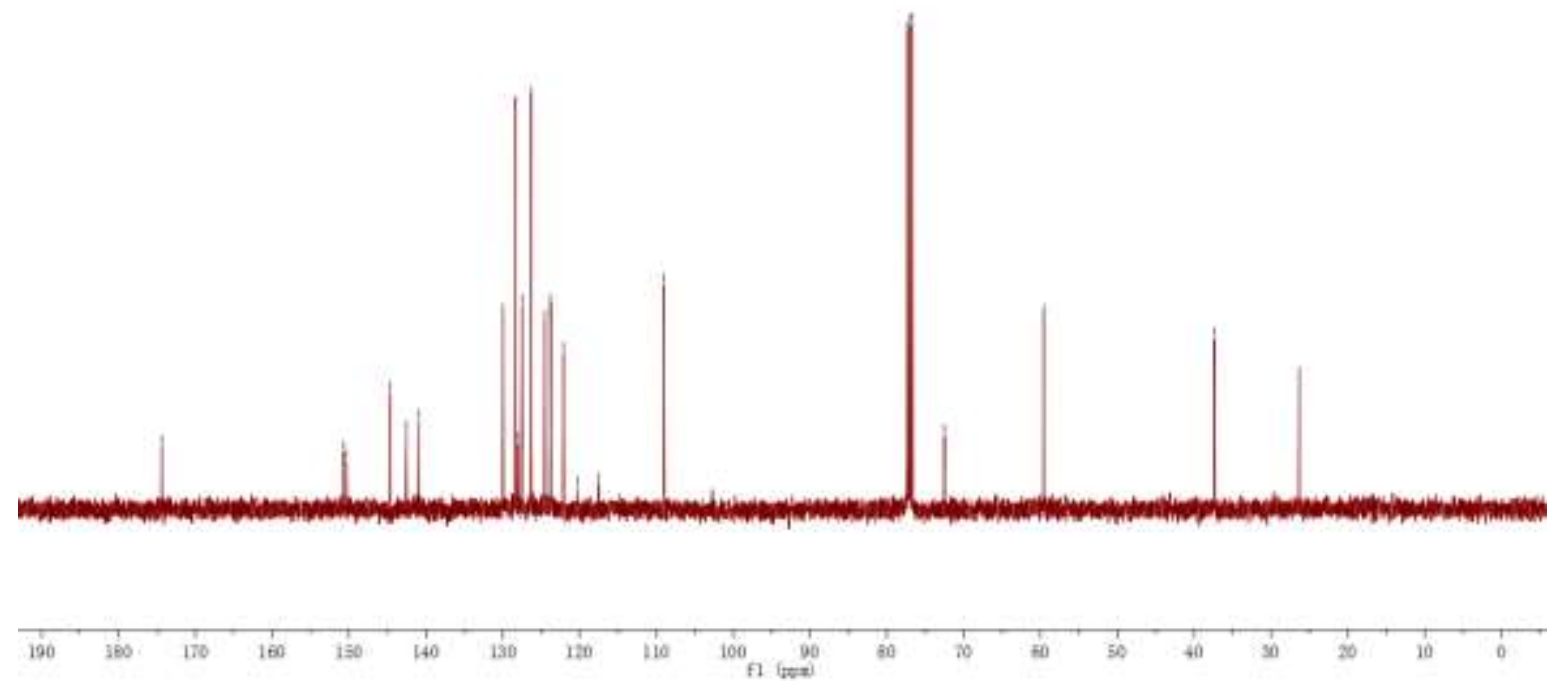

点量

EF

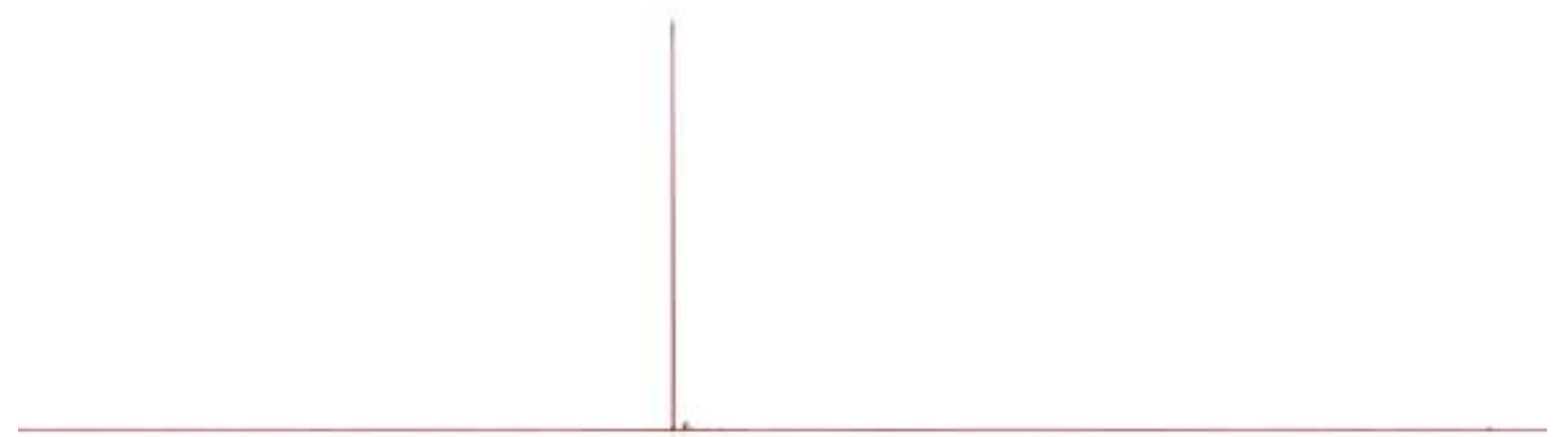

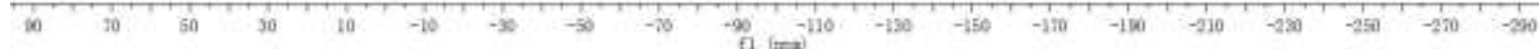


<smiles>O=C1Nc2ccccc2C1(N=CC(F)(F)F)c1ccccc1</smiles>

Compound 3ka: Yield: $74 \mathrm{mg}, 80 \%$; A yellow foam solid; Mp: 140-142 ${ }^{\circ} \mathrm{C} ;{ }^{1} \mathrm{H}$ NMR (400 MHz, Acetone $\left.-d_{6}\right) \delta 8.26-8.19(\mathrm{~m}, 1 \mathrm{H}), 7.59(\mathrm{~d}, J=7.6 \mathrm{~Hz}, 1 \mathrm{H}), 7.32-7.20(\mathrm{~m}, 8 \mathrm{H}), 7.16(\mathrm{t}, J=7.6 \mathrm{~Hz}$, 2H), $7.05(\mathrm{t}, J=7.6 \mathrm{~Hz}, 2 \mathrm{H}), 6.90(\mathrm{~d}, J=8.0 \mathrm{~Hz}, 1 \mathrm{H}), 5.55-5.45(\mathrm{~m}, 1 \mathrm{H}), 5.18(\mathrm{~d}, J=15.6 \mathrm{~Hz}, 1 \mathrm{H})$, $4.74(\mathrm{~d}, J=15.6 \mathrm{~Hz}, 1 \mathrm{H}), 4.60-4.50(\mathrm{~m}, 1 \mathrm{H}), 4.46-4.36(\mathrm{~m}, 1 \mathrm{H}), 3.64(\mathrm{t}, J=5.6 \mathrm{~Hz}, 1 \mathrm{H})$, 3.42-3.19 (m, 2H); ${ }^{13} \mathrm{C}$ NMR (100 MHz, Acetone-d) $\delta 174.9,151.5$ (q, J = 38.3 Hz), 145.1, 143.3, $142.3,136.5,130.5,129.5,129.0,129.0,128.2,128.0,127.9,127.1,125.5,124.1,123.0,122.9$, 120.3 (q, $J=373.3 \mathrm{~Hz}), 110.8,72.9,59.7,44.3,36.9 ;{ }^{19} \mathrm{~F}$ NMR (376 MHz, Chloroform- $d$ ) $\delta-66.76$ (d, $J=3.0 \mathrm{~Hz}$ ); IR (neat): v 3325, 17801612 1488, 1464, 1171, $695 \mathrm{~cm}^{-1}$; HRMS (ESI) Calcd. for $\mathrm{C}_{27} \mathrm{H}_{24} \mathrm{~F}_{3} \mathrm{~N}_{2} \mathrm{O}_{2}[\mathrm{M}+\mathrm{H}]^{+}:$465.1784, found: 465.1786 .

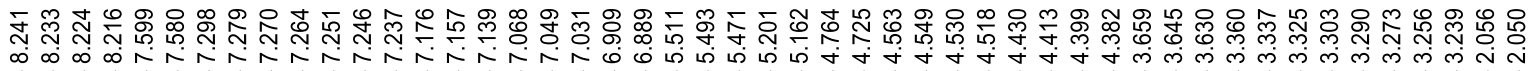

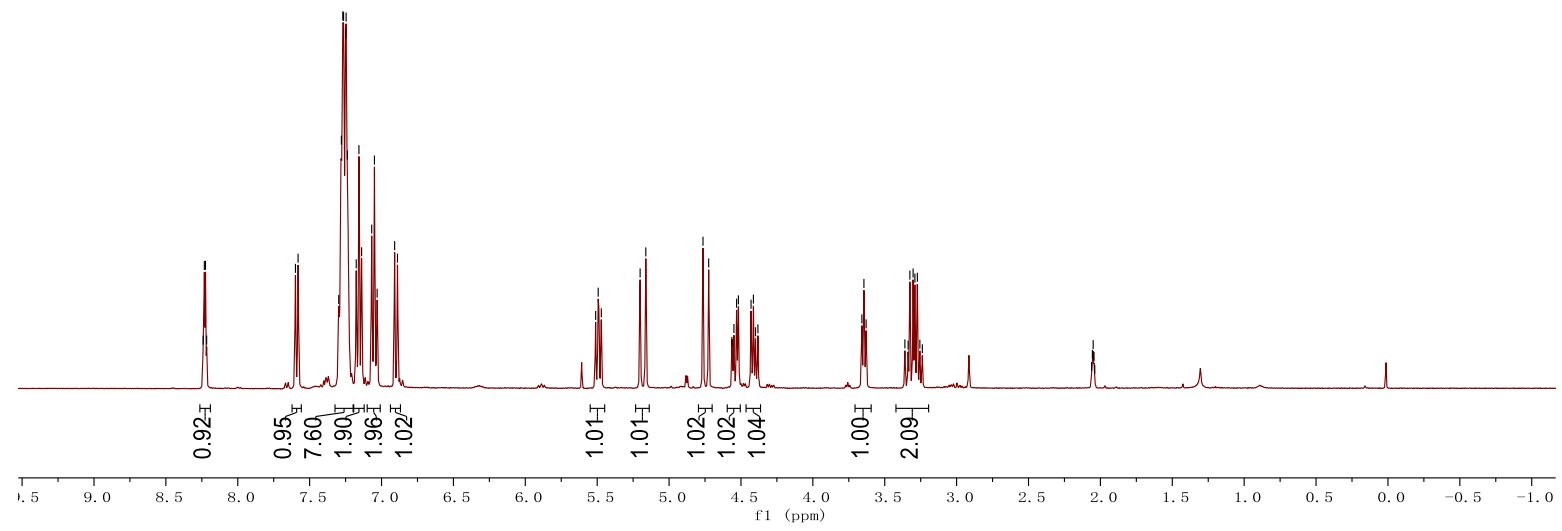



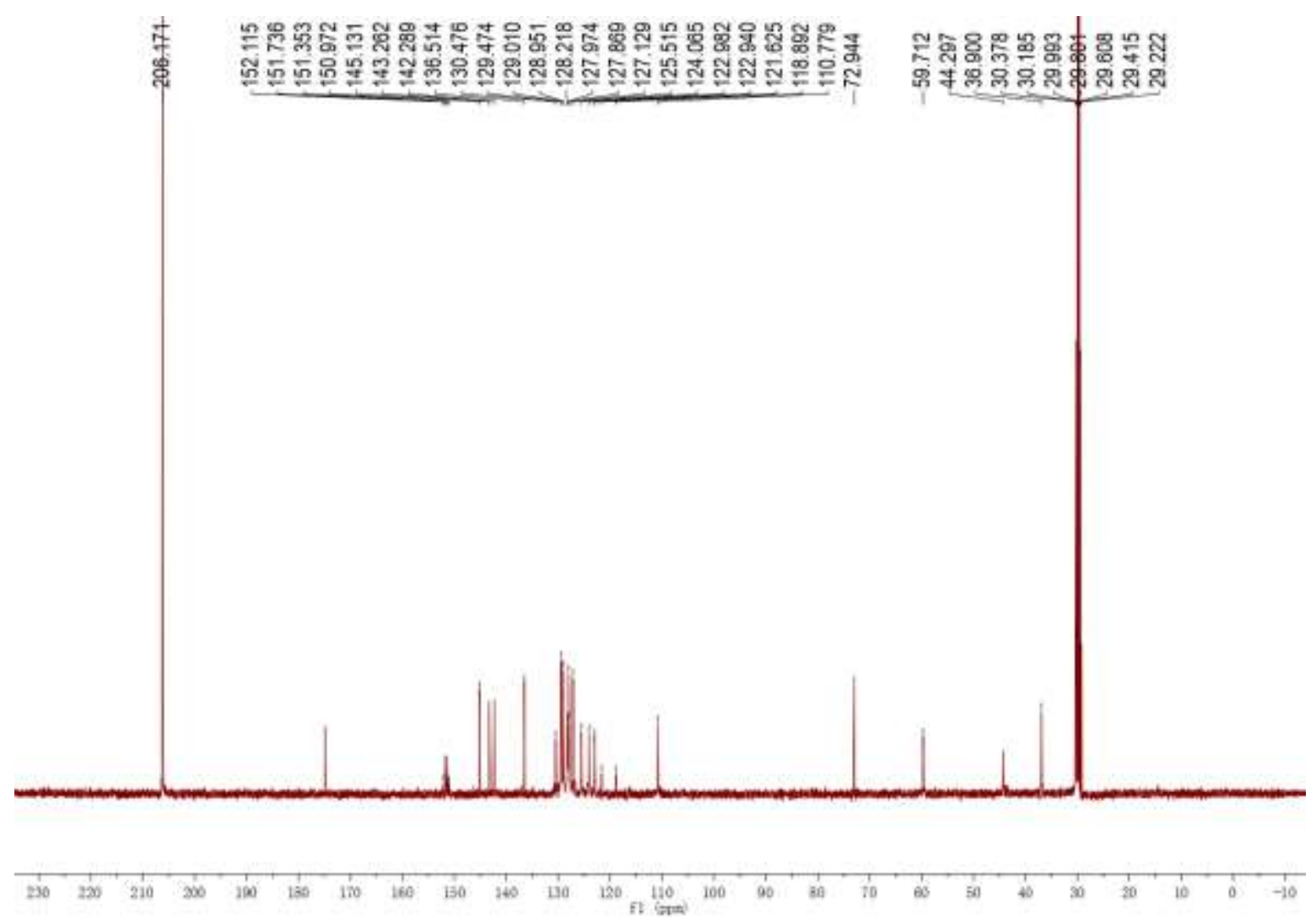

骂司

\%

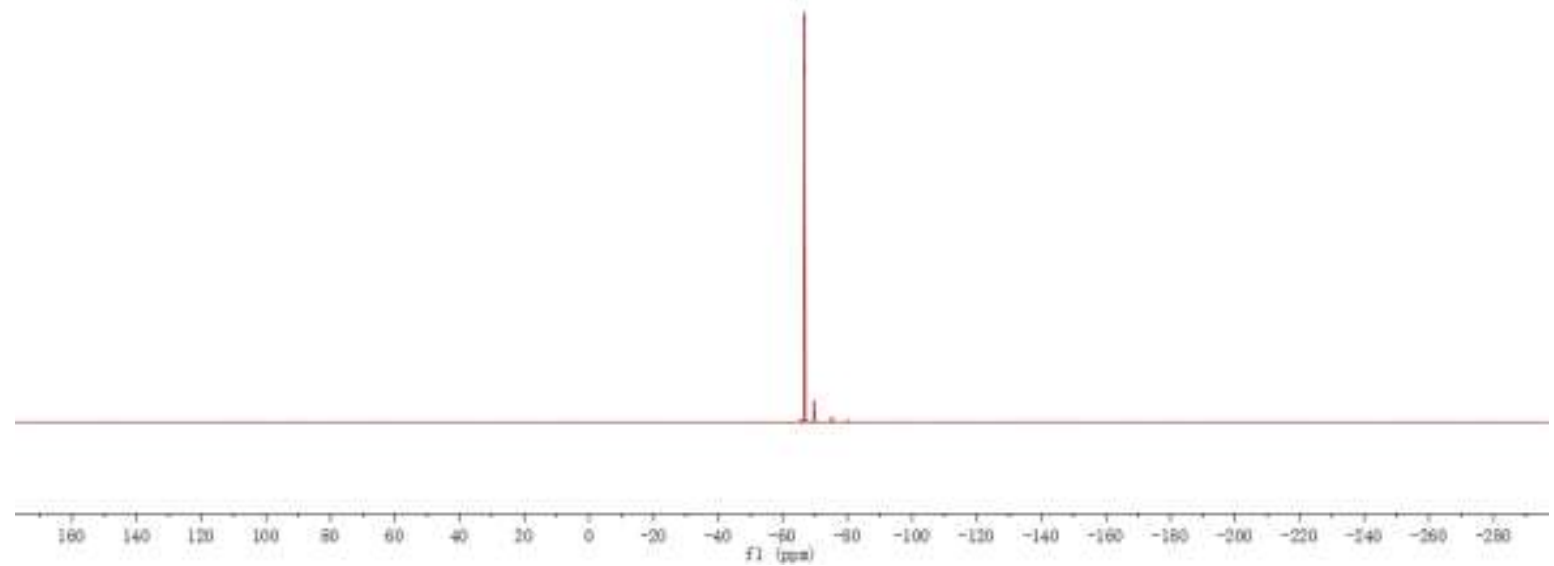




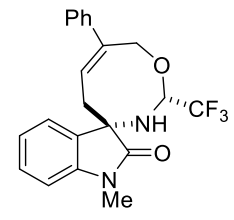

Compound 4aa: Yield: $65 \mathrm{mg}$, 85\%; A white solid; Mp: 154-156 ${ }^{\circ} \mathrm{C}$; ${ }^{1} \mathrm{H}$ NMR (400 MHz, Chloroform- $d$ ) $\delta$ 7.55-7.49 (m, 2H), 7.43-7.29 (m, 4H), 7.20-7.13 (m, 1H), 7.07-7.00 (m, 1H), 6.92-6.83 (m, 1H), 6.35-6.27 (m, 1H), 5.73-5.64 (m, 1H), $4.81(\mathrm{~d}, J=13.6 \mathrm{~Hz}, 1 \mathrm{H}), 4.64(\mathrm{~d}, J=$ $13.6 \mathrm{~Hz}, 1 \mathrm{H}), 3.22$ (s, 3H), 3.04 (brs, 1H), 2.54 (brs, 1H), 2.46 (d, J=10.4 Hz, 1H); ${ }^{13} \mathrm{C} \mathrm{NMR} \mathrm{(100}$ $\left.\mathrm{MHz}, \mathrm{CDCl}_{3}\right) \delta 177.1,142.6,141.2,130.8,129.6,128.6,127.6,126.2,125.9,123.0(\mathrm{q}, J=280.0$ $\mathrm{Hz}), 122.9,122.4,108.7,81.2(\mathrm{q}, J=5.0 \mathrm{~Hz}), 66.2,61.8,35.5,26.2 ;{ }^{19} \mathrm{~F}$ NMR $(376 \mathrm{MHz}$, Chloroform- $d$ ) $\delta$-80.28; IR (neat): $v$ 3351, 2932, 1707, 1609, 1492, 1181, 1134, 1081, 756, 746, $701 \mathrm{~cm}^{-1}$; HRMS (ESI) Calcd. for $\mathrm{C}_{21} \mathrm{H}_{20} \mathrm{~F}_{3} \mathrm{~N}_{2} \mathrm{O}_{2}[\mathrm{M}+\mathrm{H}]^{+}: 389.1471$, found: 389.1464.
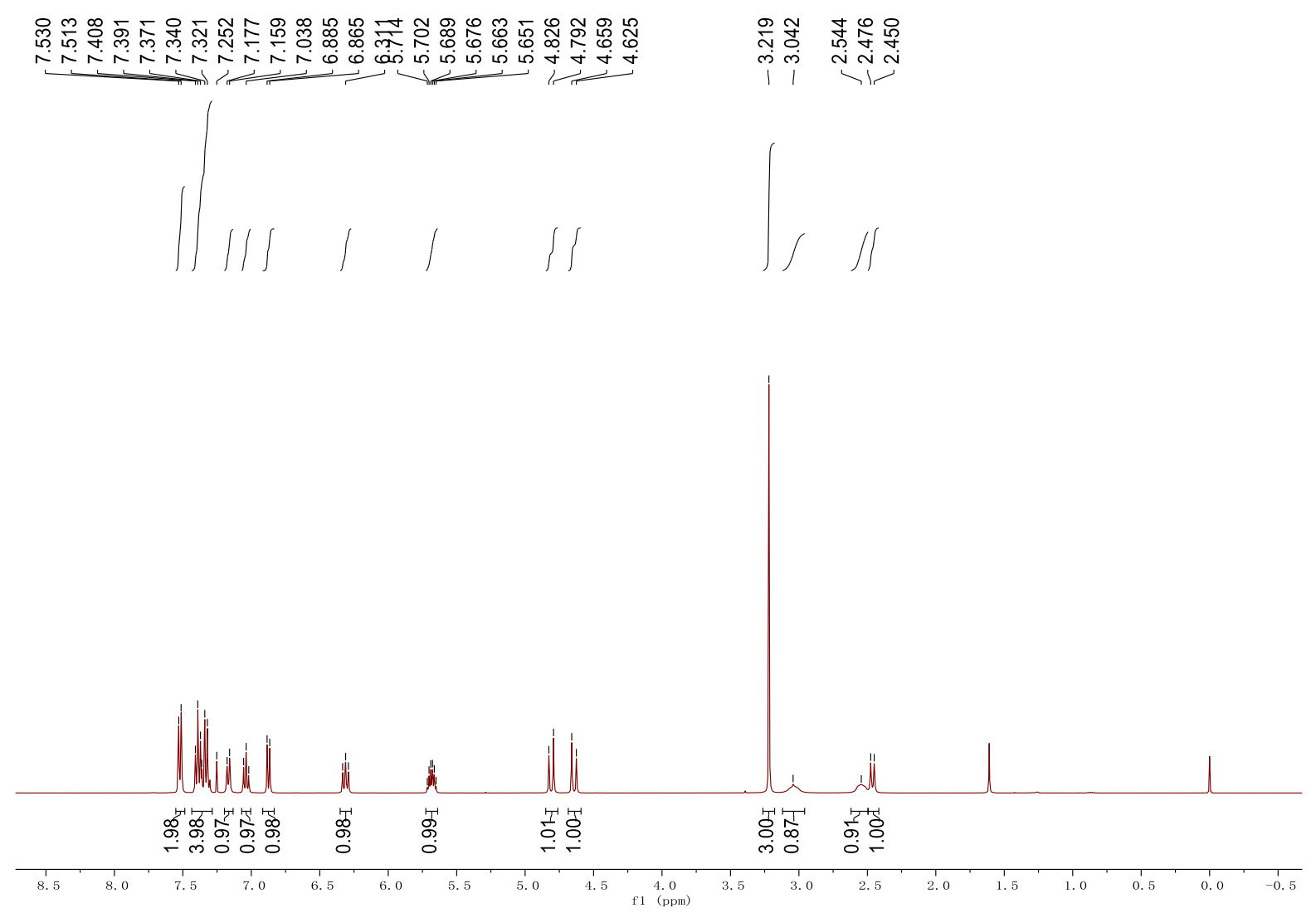


\begin{tabular}{|c|c|c|}
\hline 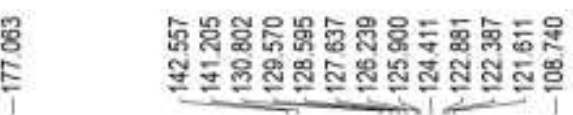 & 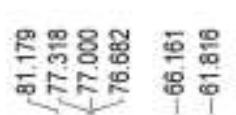 & 悉 \\
\hline
\end{tabular}
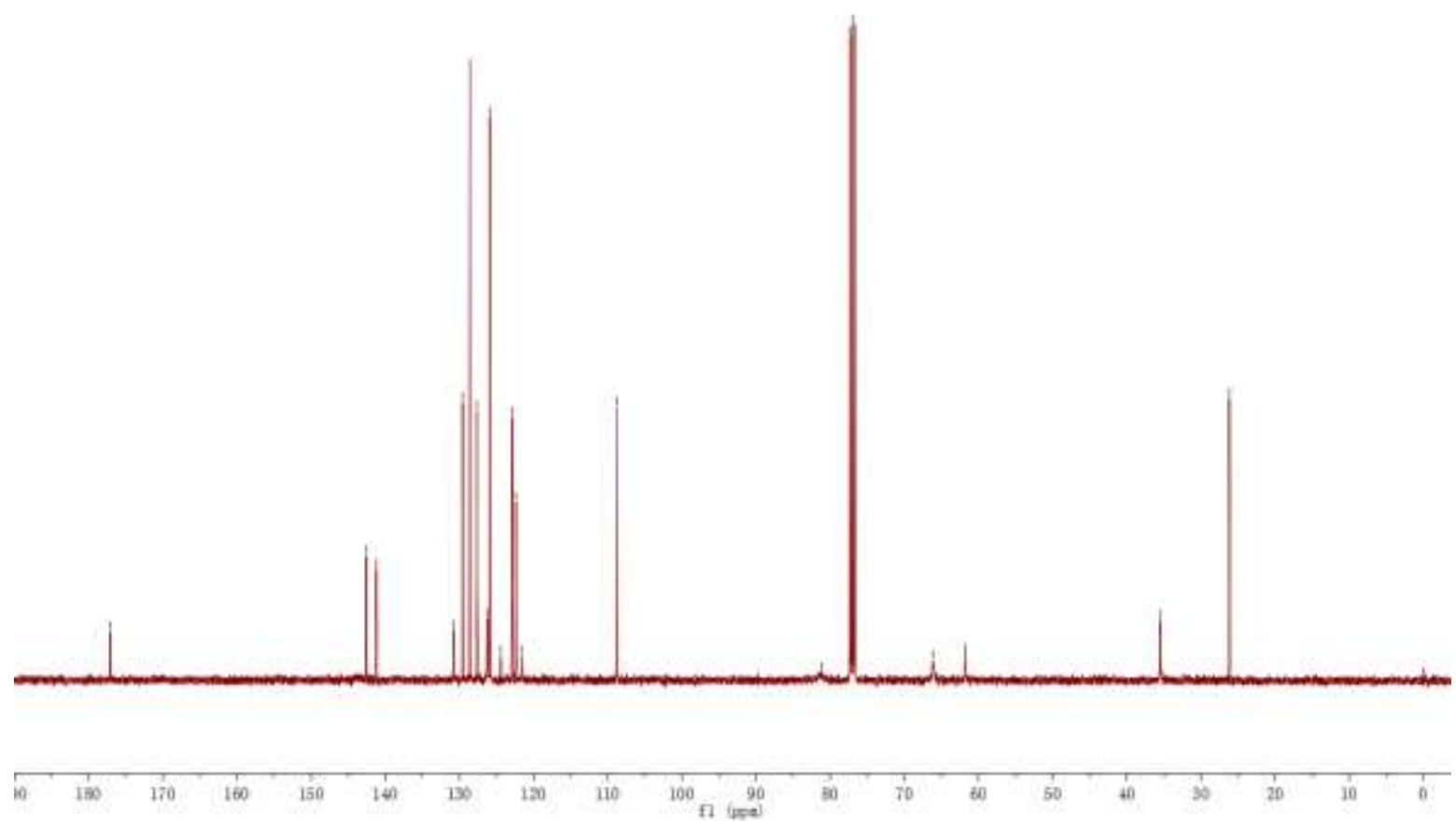

怘

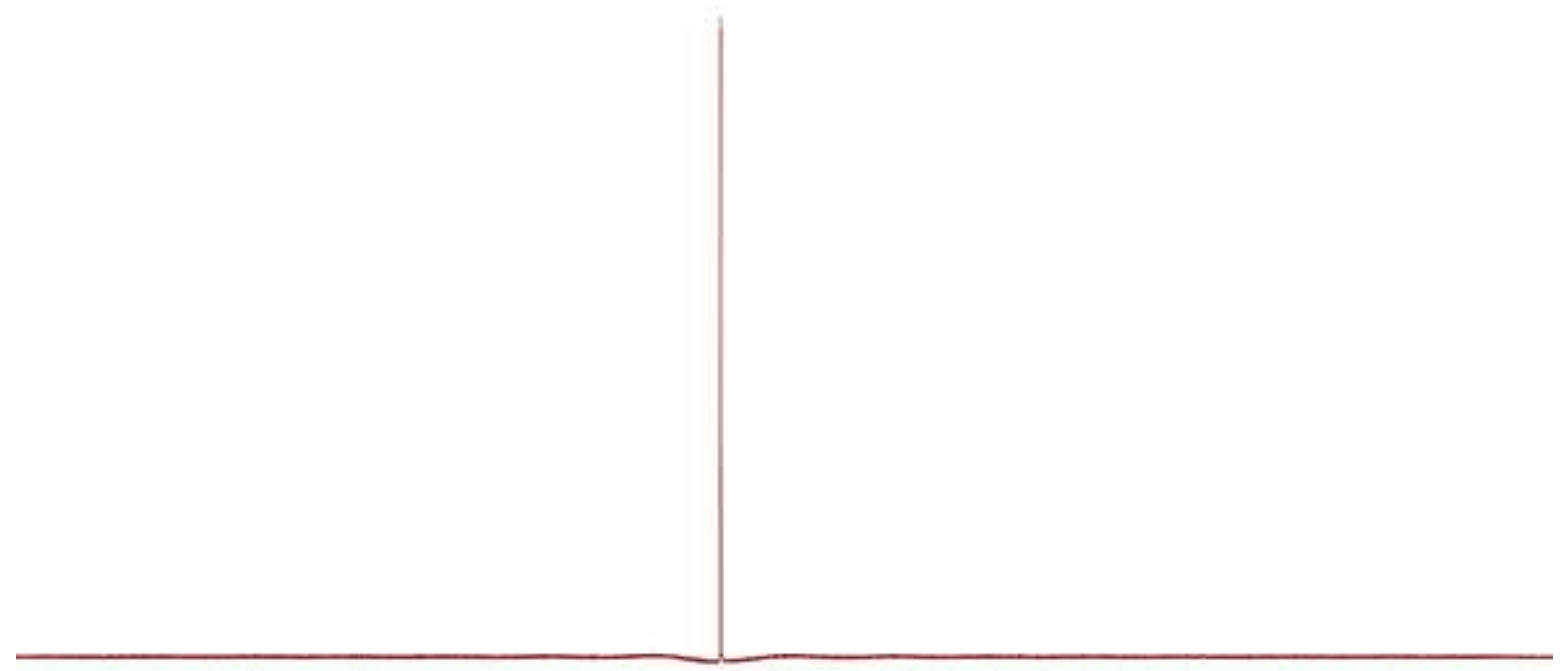

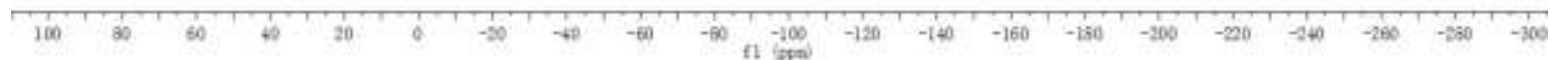


<smiles>CN1C(=O)C2(C/C=C(/c3ccccc3)COC(C(F)(F)F)N2)c2cc(Br)ccc21</smiles>

Compound 4ba: Yield: $68 \mathrm{mg}$, 73\%; A white solid; Mp: 192-193 ${ }^{\circ} \mathrm{C}$; ${ }^{1} \mathrm{H}$ NMR (400 MHz, Chloroform-d) $\delta$ 7.54-7.49 (m, 2H), $7.47(\mathrm{dd}, J=8.4 \mathrm{~Hz}, J=2.0 \mathrm{~Hz}, 1 \mathrm{H}), 7.43-7.36(\mathrm{~m}, 2 \mathrm{H})$, 7.35-7.29 (m, 2H), $6.76(\mathrm{~d}, J=8.4 \mathrm{~Hz}, 1 \mathrm{H}), 6.28(\mathrm{t}, J=8.0 \mathrm{~Hz}, 1 \mathrm{H}), 5.73-5.60(\mathrm{~m}, 1 \mathrm{H}), 4.80(\mathrm{~d}, J$ $=13.6 \mathrm{~Hz}, 1 \mathrm{H}), 4.64$ (d, $J=13.6 \mathrm{~Hz}, 1 \mathrm{H}), 3.19$ (s, 3H), 2.99 (brs, 1H), 2.57 (brs, 1H), 2.45 (d, $J=$ $9.6 \mathrm{~Hz}, 1 \mathrm{H}) ;{ }^{13} \mathrm{C} \mathrm{NMR}\left(100 \mathrm{MHz}, \mathrm{CDCl}_{3}\right) \delta 176.4,141.6,140.9,132.7,132.4,128.6,127.8,125.9$, $125.7,125.4,122.9(\mathrm{q}, J=280.1 \mathrm{~Hz}), 115.4,110.2,80.7(\mathrm{q}, J=4.0 \mathrm{~Hz}), 66.0,61.7,35.5,26.3 ;{ }^{19} \mathrm{~F}$ NMR (376 MHz, Chloroform- $d$ ) $\delta$-80.23; IR (neat): v 3364, 2921, 1800, 1718, 1187, 1087, 1065, 739, 730, $699 \mathrm{~cm}^{-1}$; HRMS (ESI) Calcd. for $\mathrm{C}_{21} \mathrm{H}_{19} \mathrm{BrF}_{3} \mathrm{~N}_{2} \mathrm{O}_{2}[\mathrm{M}+\mathrm{H}]^{+}:$467.0577, found: 467.0576 .

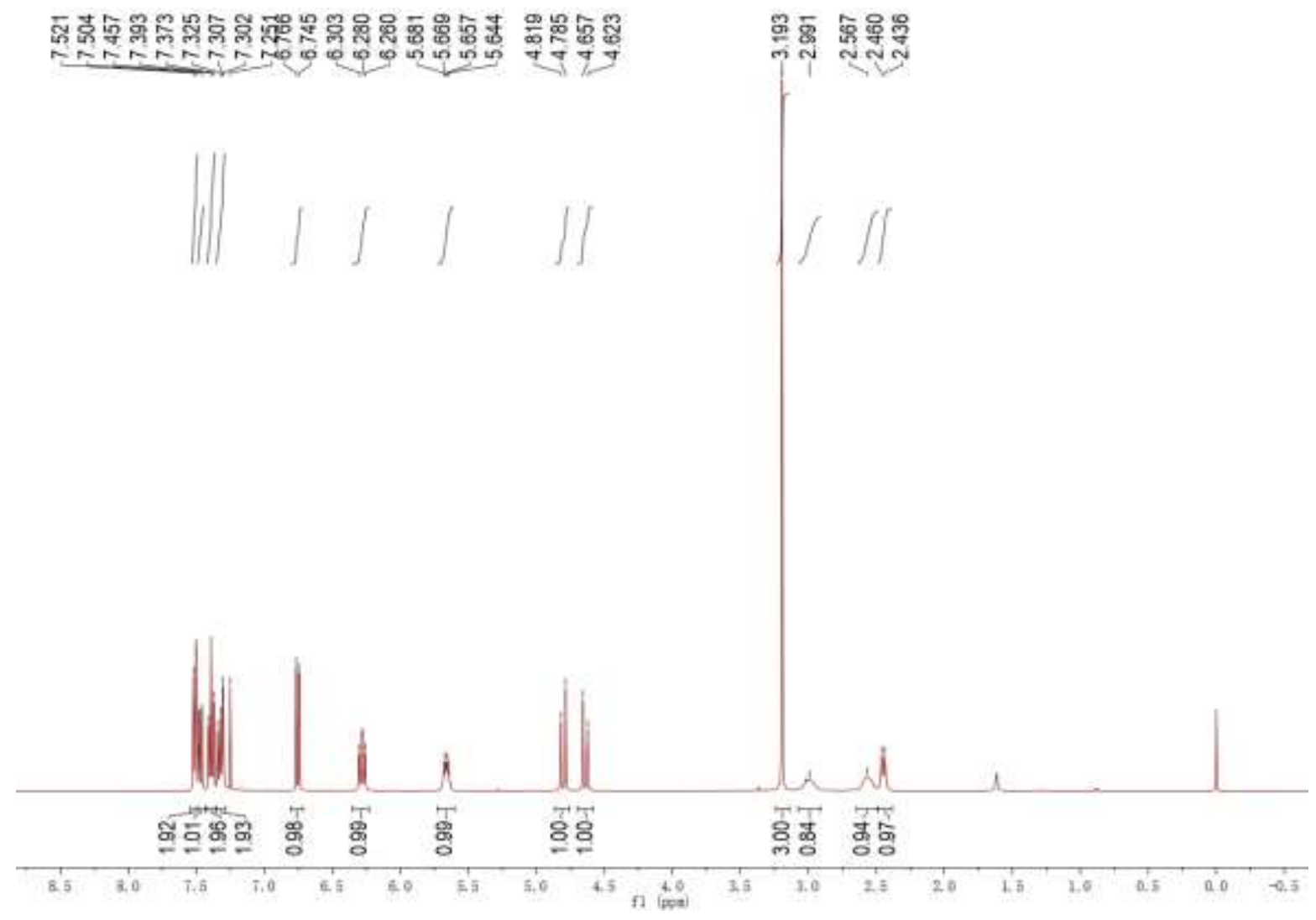




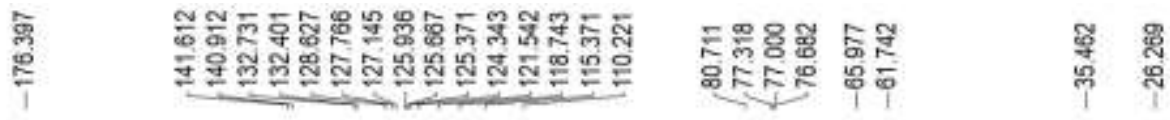
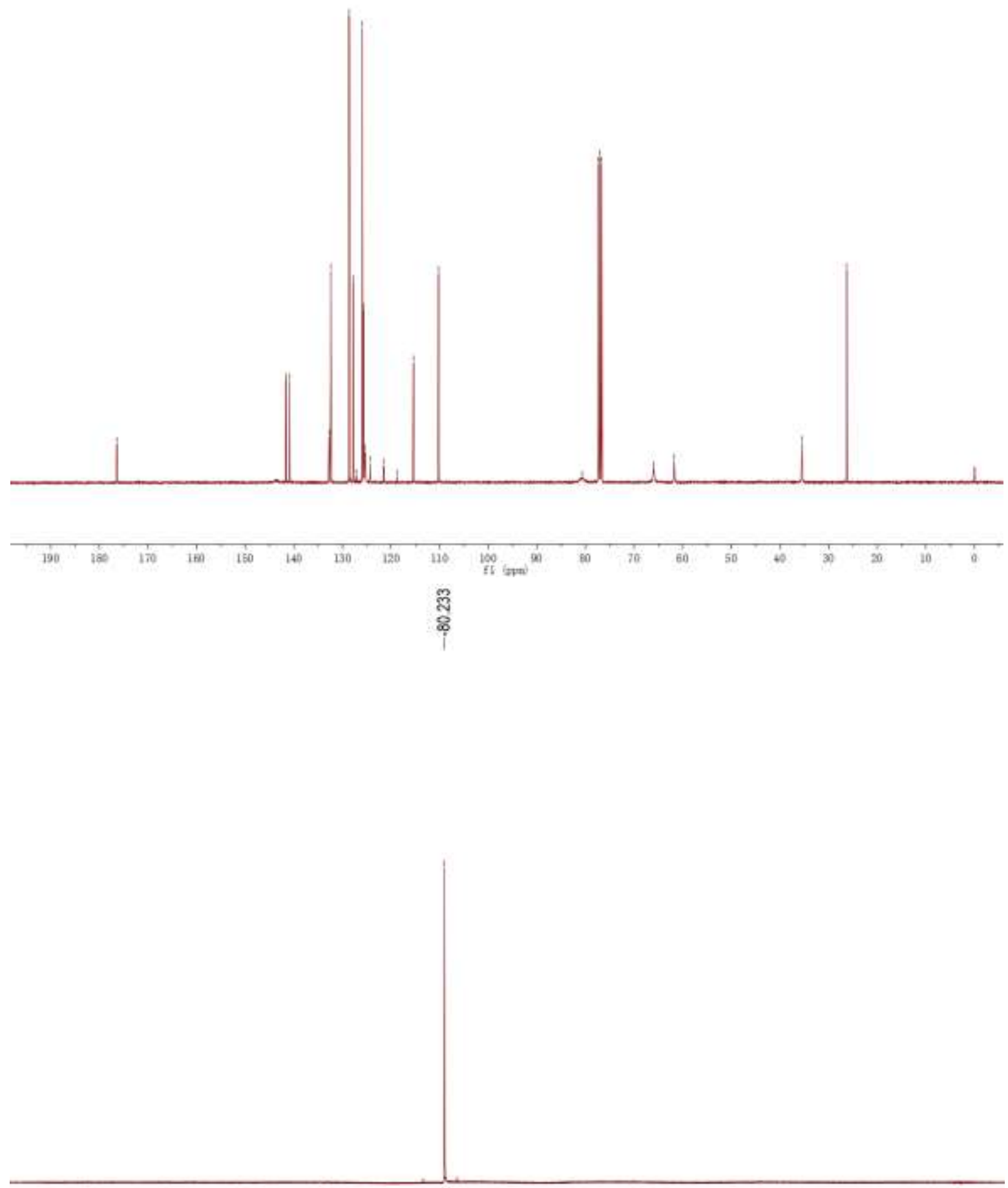

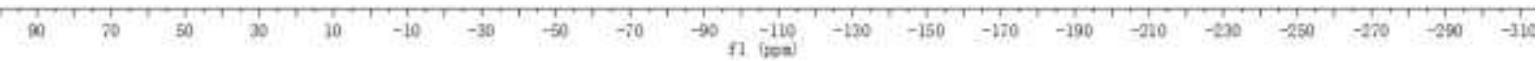


<smiles></smiles>

Compound 4ca: Yield: $69 \mathrm{mg}$, 82\%; A white solid; Mp: 186-188 ${ }^{\circ} \mathrm{C}$; ${ }^{1} \mathrm{H}$ NMR (400 MHz, Chloroform- $d$ ) $\delta$ 7.57-7.47 (m, 2H), 7.46-7.28 (m, 4H), $7.17(\mathrm{~s}, 1 \mathrm{H}), 6.81(\mathrm{~d}, J=8.4 \mathrm{~Hz}, 1 \mathrm{H}), 6.29$ (t, $J=8.4 \mathrm{~Hz}, 1 \mathrm{H}), 5.73-5.61(\mathrm{~m}, 1 \mathrm{H}), 4.81(\mathrm{~d}, J=13.6 \mathrm{~Hz}, 1 \mathrm{H}), 4.65(\mathrm{~d}, J=13.6 \mathrm{~Hz}, 1 \mathrm{H}), 3.21(\mathrm{~s}$, 3H), 3.02 (brs, 1H), 2.56 (brs, 1H), 2.45 (d, $J=10.0 \mathrm{~Hz}, 1 \mathrm{H}) ;{ }^{13} \mathrm{C} \mathrm{NMR}\left(100 \mathrm{MHz}, \mathrm{CDCl}_{3}\right) \delta 176.5$, $141.1,140.9$, 132.3, 129.5, 128.6, 128.2, 127.8, 125.9, 125.4, 123.0, 122.9 (q, $J=280.5 \mathrm{~Hz}), 109.7$, 80.7 (q, $J=3.0 \mathrm{~Hz}), 66.1,61.8,35.4,26.3 ;{ }^{19} \mathrm{~F}$ NMR (376 MHz, Chloroform- $d$ ) $\delta$-80.25; IR (neat): $v$ 3369, 2924, 1719, 1488, 1284, 1187, 1166, 1039, 815, $699 \mathrm{~cm}^{-1}$; HRMS (ESI) Calcd. for $\mathrm{C}_{21} \mathrm{H}_{19} \mathrm{ClF}_{3} \mathrm{~N}_{2} \mathrm{O}_{2}[\mathrm{M}+\mathrm{H}]^{+}:$423.1082, found: 423.1084 .

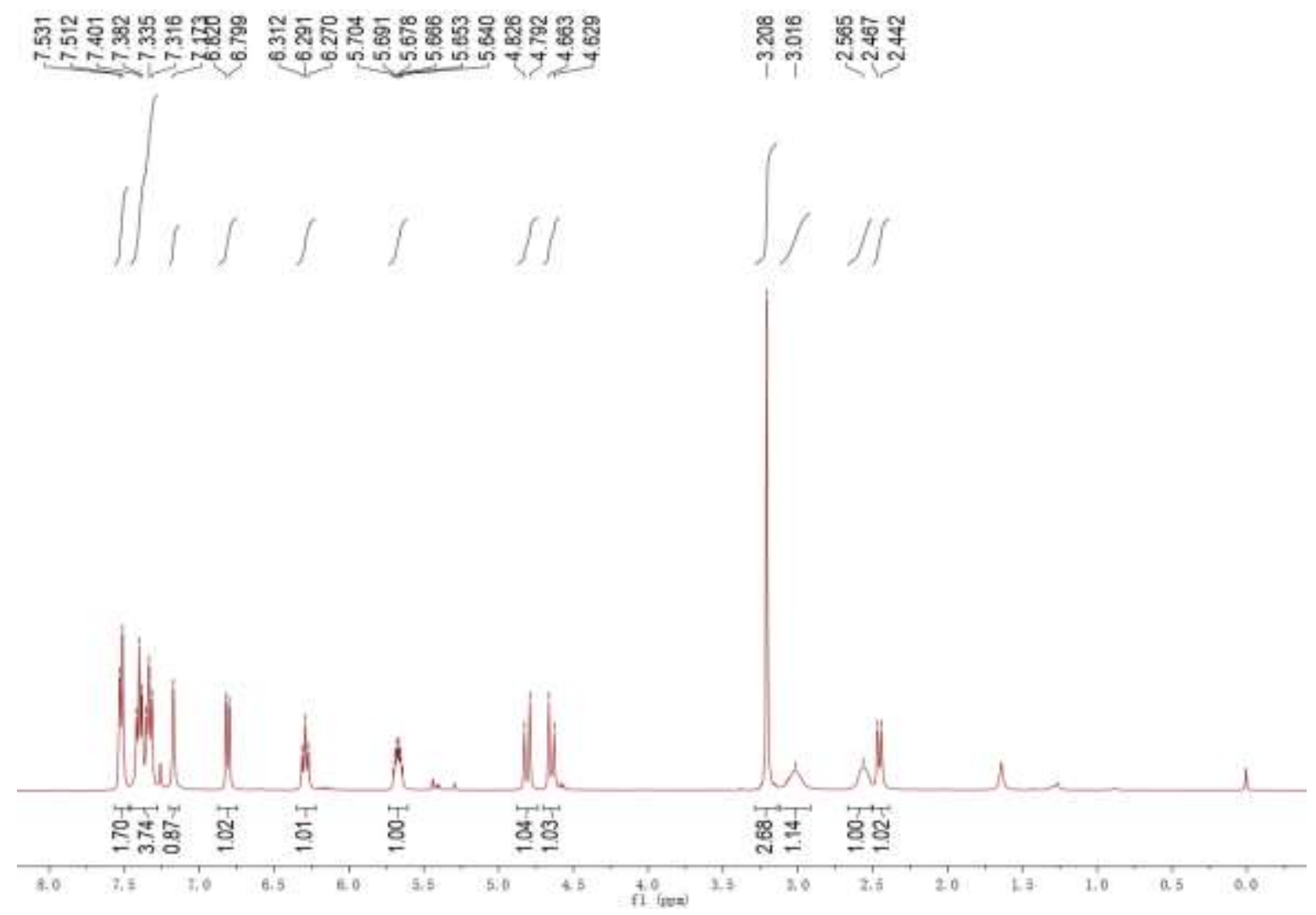




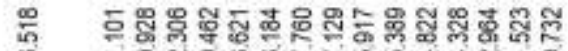

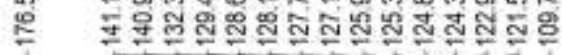

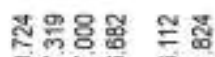

OFFः :

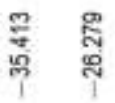
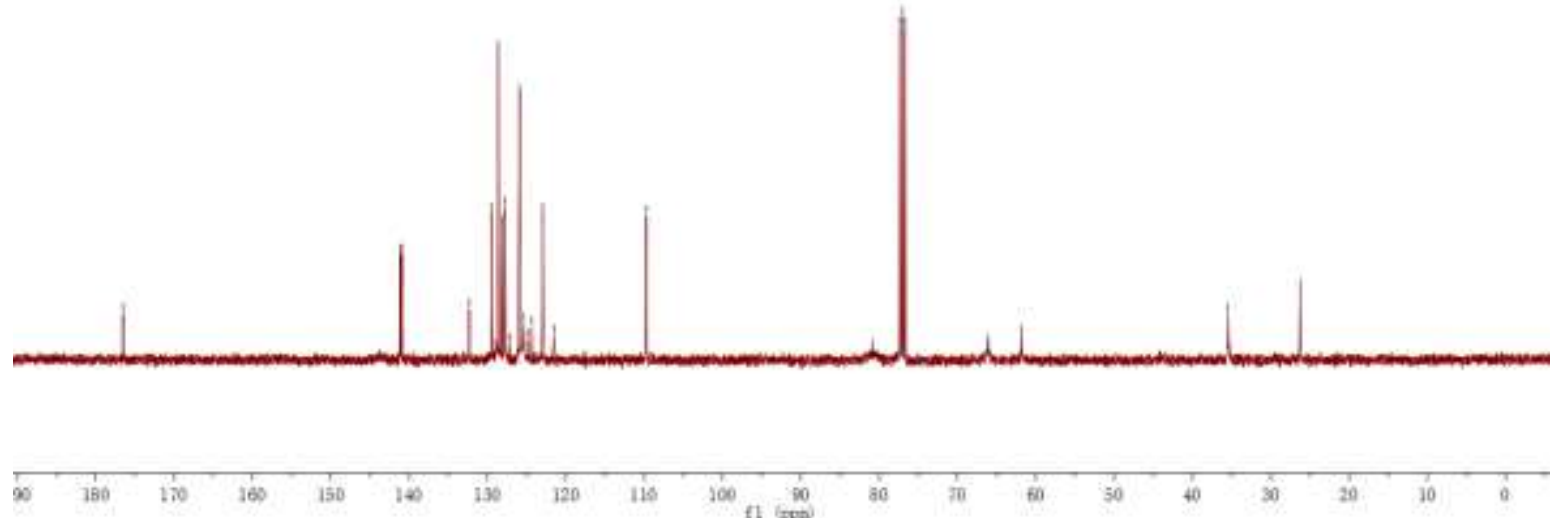

ำ

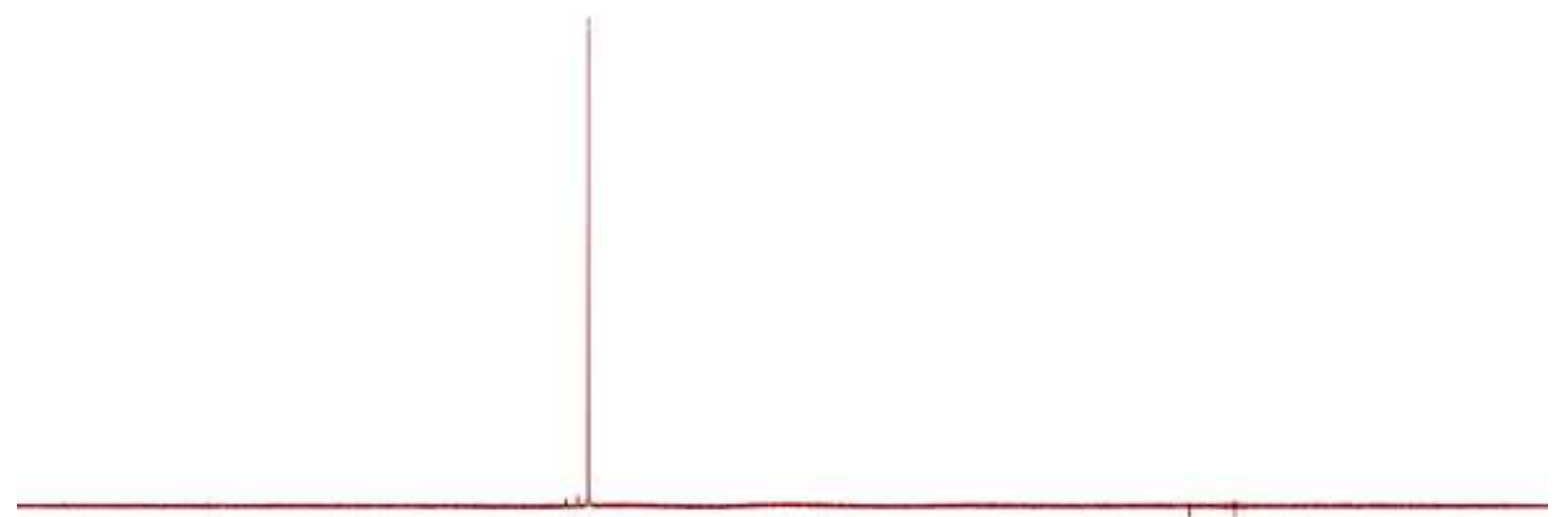

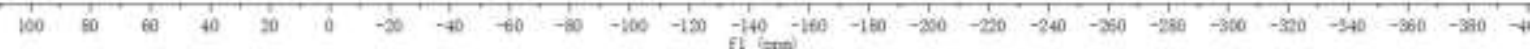




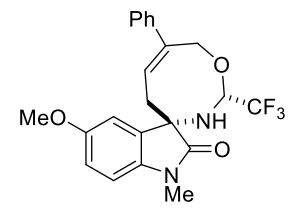

Compound 4da: Yield: $64 \mathrm{mg}$, 77\%; A white solid; Mp: 192-194 ${ }^{\circ} \mathrm{C}$; ${ }^{1} \mathrm{H}$ NMR (400 MHz, Chloroform- $d$ ) $\delta$ 7.55-7.47 (m, 2H), 7.42-7.34 (m, 2H), 7.34-7.27 (m, 1H), 6.87-6.82 (m, 1H), 6.82-6.74 (m, 2H), $6.31(\mathrm{t}, J=8.4 \mathrm{~Hz}, 1 \mathrm{H}), 5.75-5.64(\mathrm{~m}, 1 \mathrm{H}), 4.81(\mathrm{~d}, J=13.6 \mathrm{~Hz}, 1 \mathrm{H}), 4.64(\mathrm{~d}, J$ $=13.6 \mathrm{~Hz}, 1 \mathrm{H}), 3.75(\mathrm{~s}, 3 \mathrm{H}), 3.19(\mathrm{~s}, 3 \mathrm{H}), 3.01$ (brs, $1 \mathrm{H}), 2.55$ (brs, 1H), 2.45 (d, J = $10.4 \mathrm{~Hz}, 1 \mathrm{H})$; ${ }^{13} \mathrm{C}$ NMR $\left(100 \mathrm{MHz} \mathrm{CDCl}_{3}\right) \delta 176.7,156.1,141.1,135.9,132.0,128.6,127.6,126.1,125.9,123.0$ $(\mathrm{q}, J=280.1 \mathrm{~Hz}), 113.0,110.5,109.0,80.8(\mathrm{q}, J=5.0 \mathrm{~Hz}), 66.0,62.0,55.8,35.5,26.2 ;{ }^{19} \mathrm{~F}$ NMR (376 MHz, Chloroform-d) $\delta$-80.25; IR (neat): v 3298, 2947, 1684, 1498, 1230, 1166, 1100, 1081, 855, $676 \mathrm{~cm}^{-1}$; HRMS (ESI) Calcd. for $\mathrm{C}_{22} \mathrm{H}_{22} \mathrm{~F}_{3} \mathrm{~N}_{2} \mathrm{O}_{2}[\mathrm{M}+\mathrm{H}]^{+}:$419.1577, found: 419.1576.

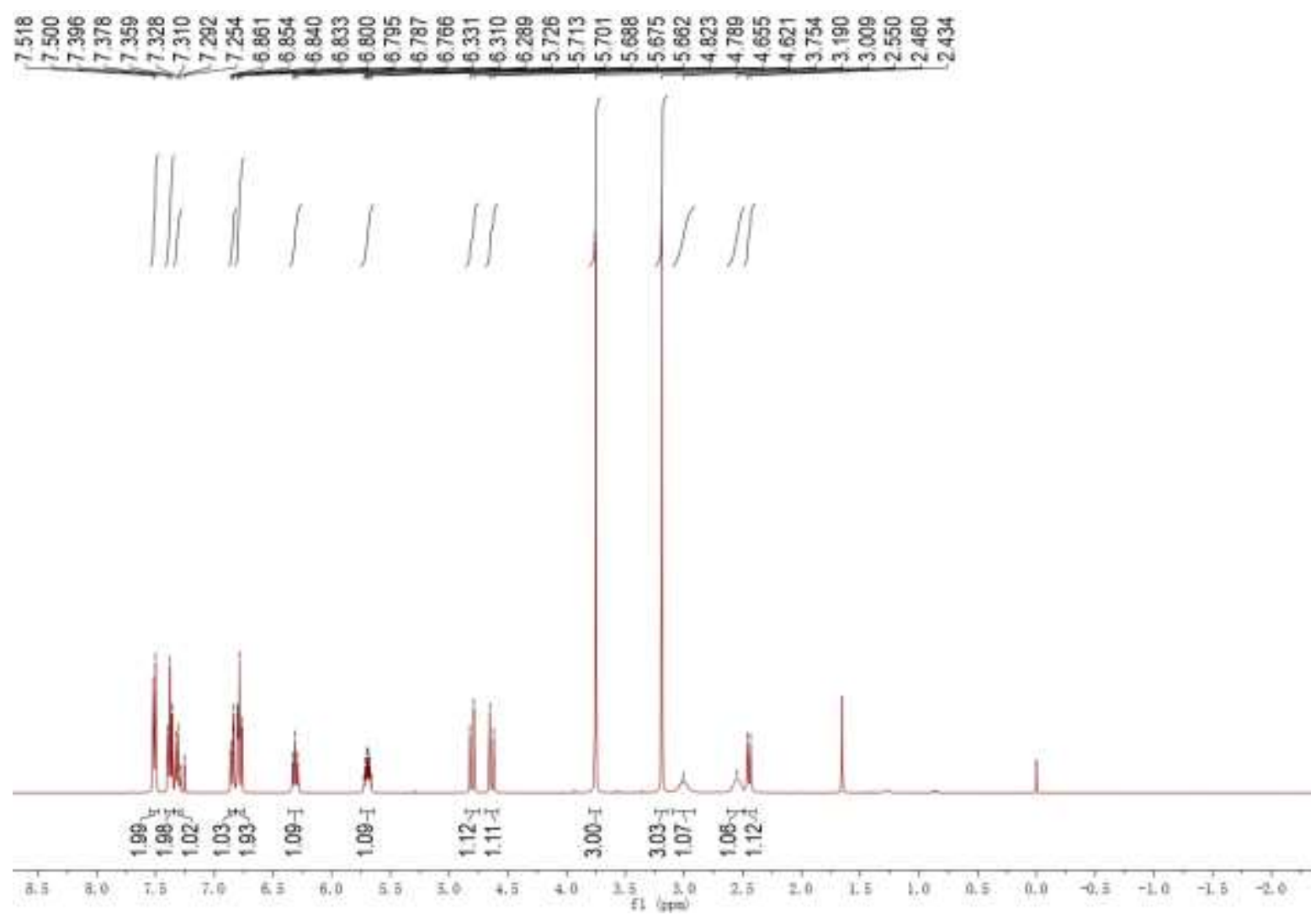




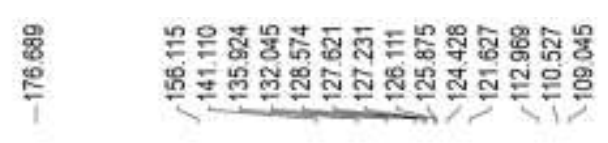

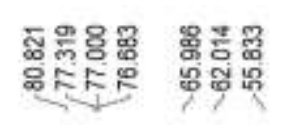

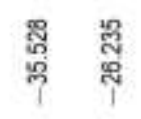
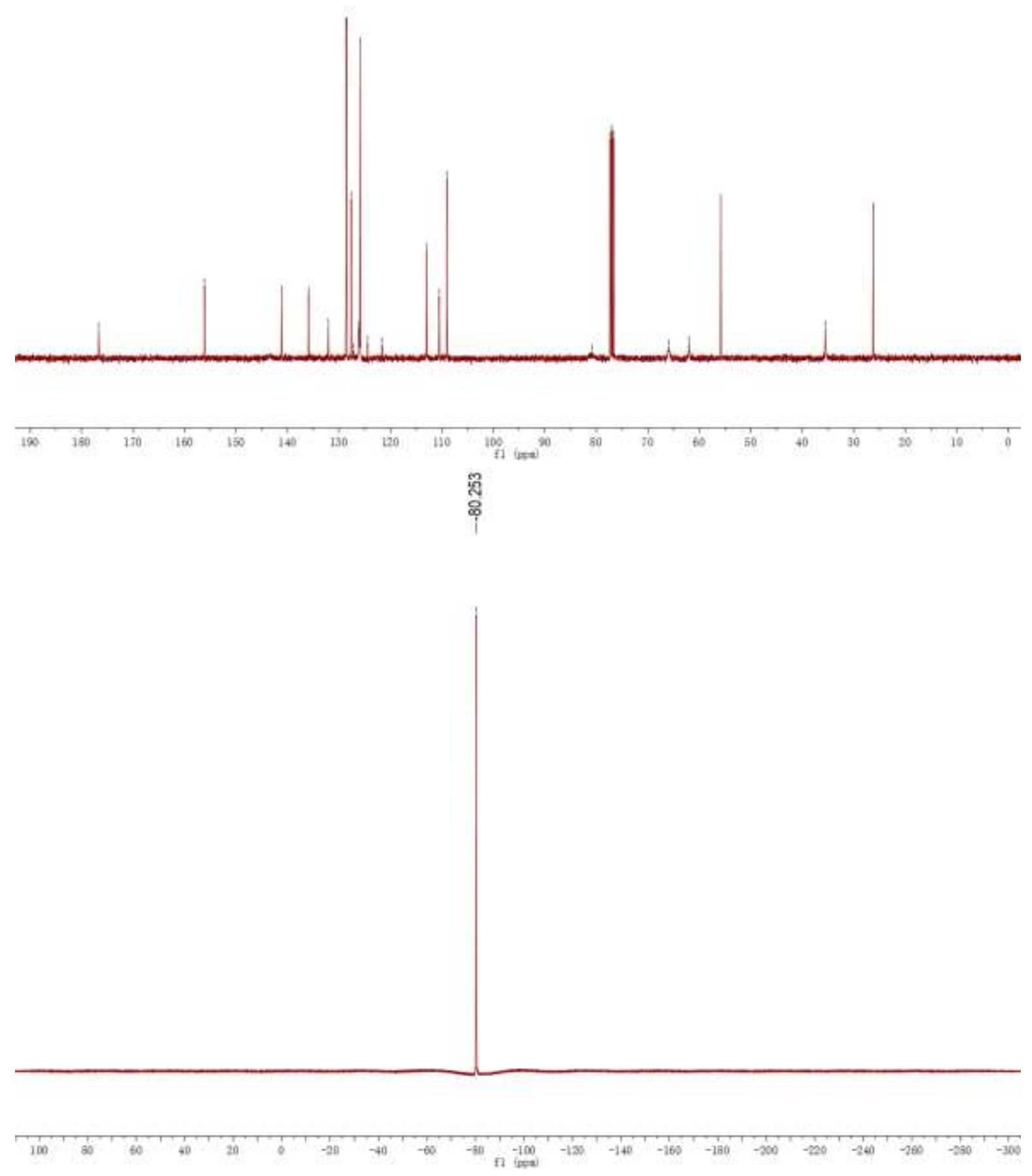

S17 
<smiles>Cc1ccc(C2(C(=O)Nc3ccccc3)C/C=C(\c3ccccc3)COC(C(F)(F)F)N2)c([N+](=O)[O-])c1</smiles>

Compound 4ea: Yield: $65 \mathrm{mg}, 81 \%$; A white solid; Mp: 148-150 ${ }^{\circ} \mathrm{C}$; ${ }^{1} \mathrm{H}$ NMR (400 MHz, Chloroform- $d$ ) $\delta$ 7.57-7.49 (m, 2H), 7.43-7.35 (m, 2H), 7.35-7.28 (m, 1H), 7.14 (d, J = 8.0 Hz, 1H), $6.99(\mathrm{~s}, 1 \mathrm{H}), 6.76(\mathrm{~d}, J=8.0 \mathrm{~Hz}, 1 \mathrm{H}), 6.33(\mathrm{t}, J=8.0 \mathrm{~Hz}, 1 \mathrm{H}), 5.69(\mathrm{~m}, 1 \mathrm{H}), 4.81(\mathrm{~d}, J=13.6$ Hz, 1H), 4.64 (d, J=13.6 Hz, 1H), 3.19 (s, 3H), 2.97 (brs, 1H), 2.57 (brs, 1H), 2.45 (d, J = 10.0 Hz, 1H), $2.31(\mathrm{~s}, 3 \mathrm{H}) ;{ }^{13} \mathrm{C} \mathrm{NMR}\left(100 \mathrm{MHz}, \mathrm{CDCl}_{3}\right) \delta 176.9,141.2,140.1,132.6,130.9,129.7,128.6$, 127.6, 126.4, 125.9, 123.1, 123.0 (q, $J=280.0 \mathrm{~Hz}), 108.5,80.7$ (q, $J=5.0 \mathrm{~Hz}), 65.9,61.8,35.7$, 26.2, 21.1; ${ }^{19}$ F NMR (376 MHz, Chloroform- $d$ ) $\delta$-80.32; IR (neat): v 3371, 3337, 2934, 1702, 1497 , 1168, 1157, 818, 774, $710 \mathrm{~cm}^{-1}$; HRMS (ESI) Calcd. for $\mathrm{C}_{22} \mathrm{H}_{22} \mathrm{~F}_{3} \mathrm{~N}_{2} \mathrm{O}_{2}[\mathrm{M}+\mathrm{H}]^{+}:$403.1628, found: 403.1628 .

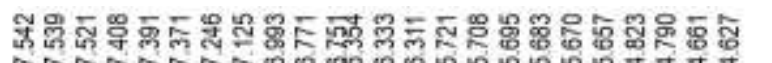

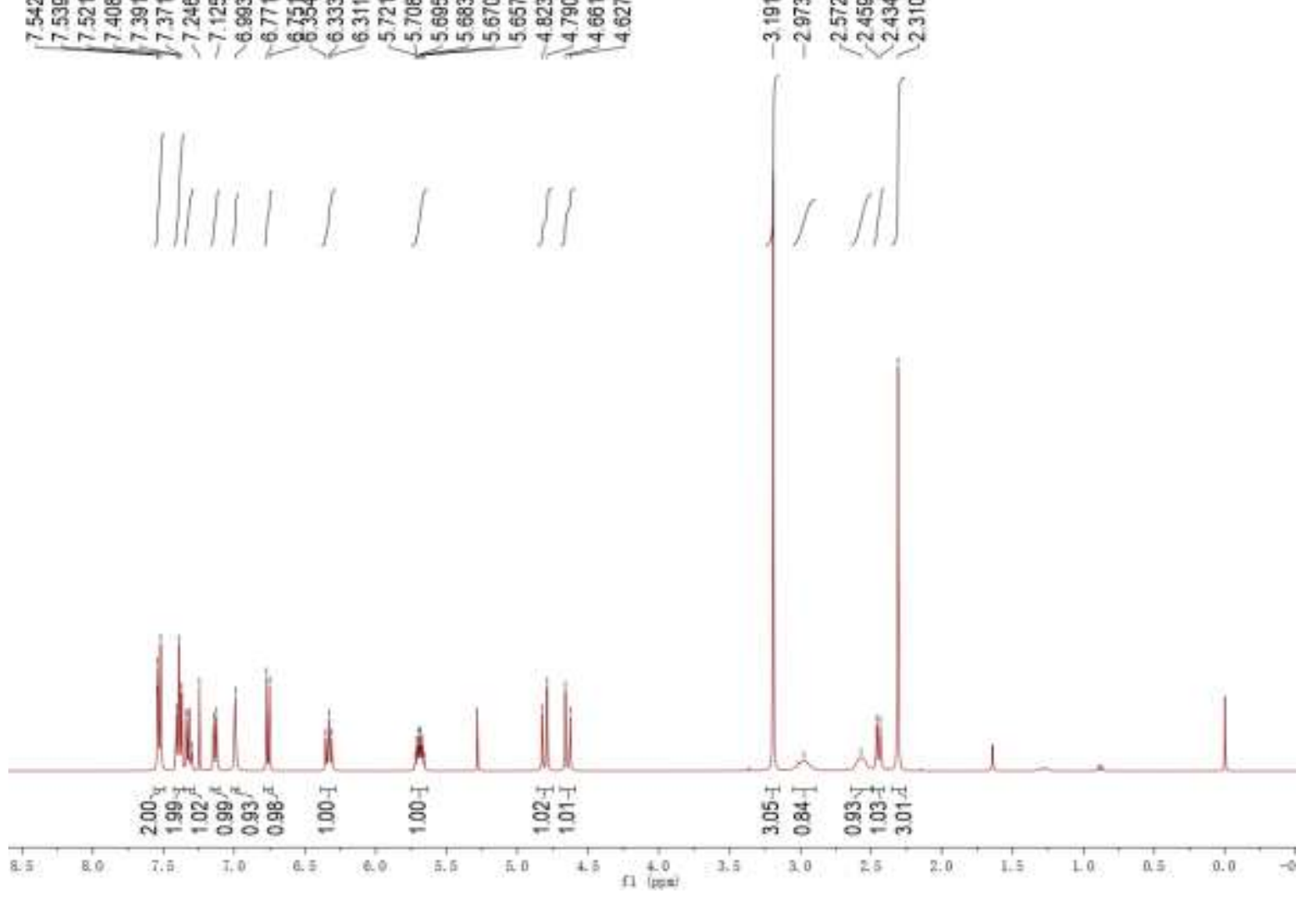



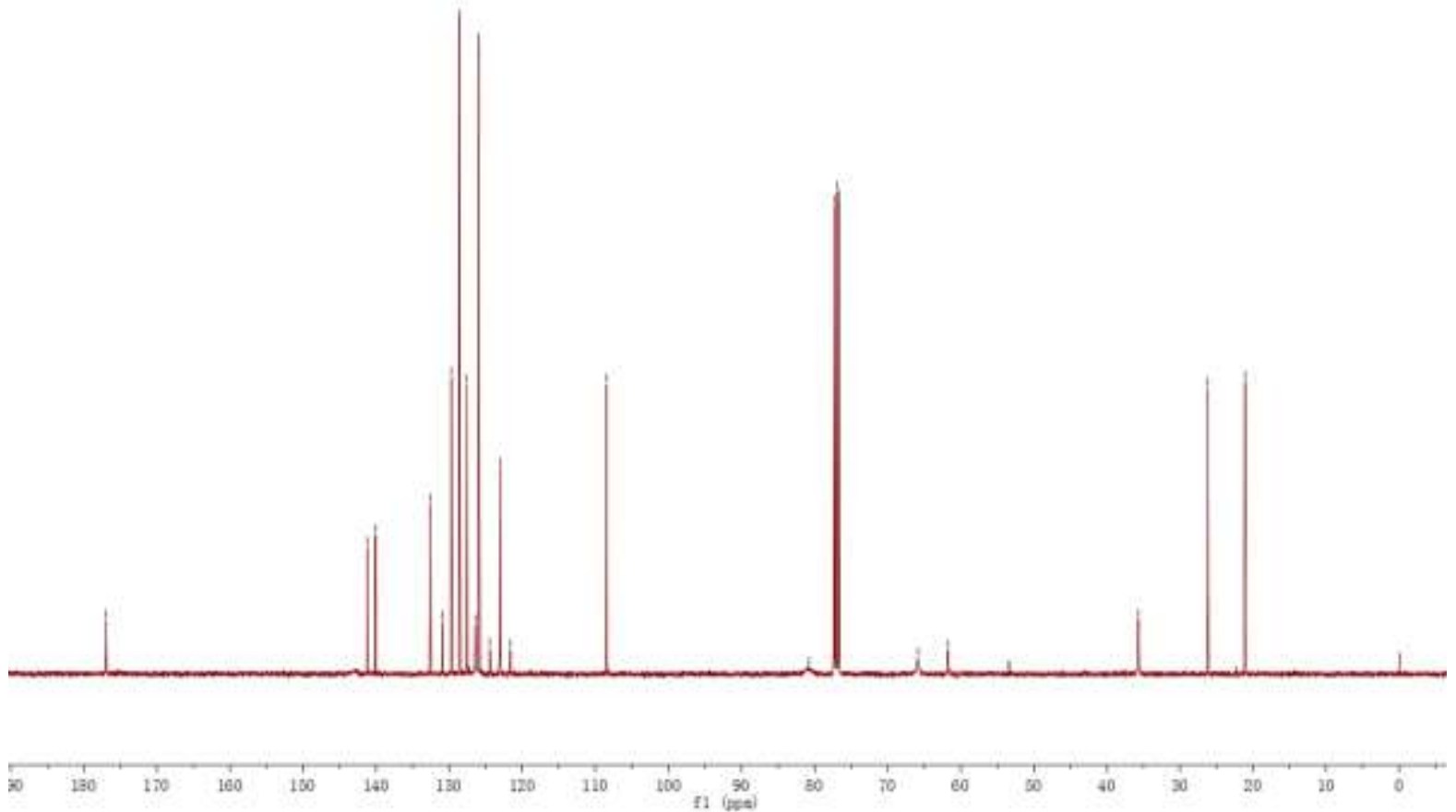

लำ

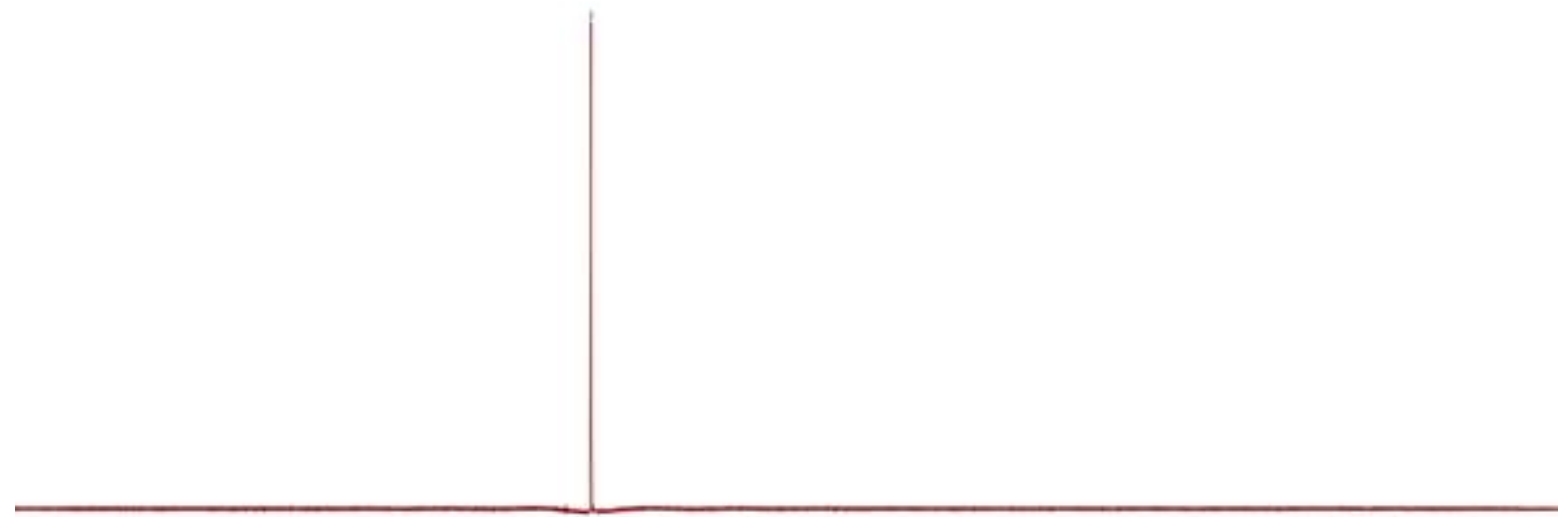

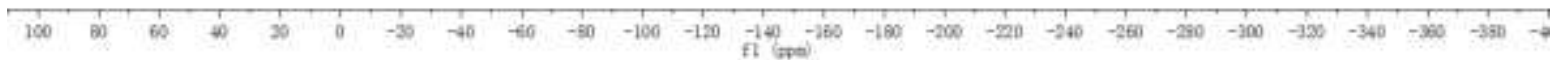




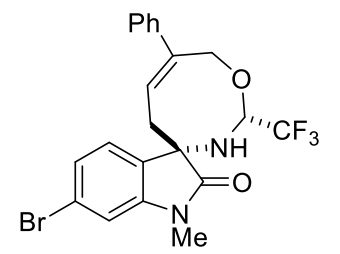

Compound 4fa: Yield: $62 \mathrm{mg}$, 66\%; A white solid; Mp: 206-208 ${ }^{\circ} \mathrm{C}$; ${ }^{1} \mathrm{H}$ NMR (400 MHz, Acetone- $\left.d_{6}\right) \delta 7.60-7.53(\mathrm{~m}, 2 \mathrm{H}), 7.47(\mathrm{~d}, J=8.4 \mathrm{~Hz}, 1 \mathrm{H}), 7.43-7.36(\mathrm{~m}, 2 \mathrm{H}), 7.35-7.28(\mathrm{~m}, 1 \mathrm{H})$, 7.26-7.21 (m, 2H), $6.40(\mathrm{t}, J=8.4 \mathrm{~Hz}, 1 \mathrm{H}), 5.78-5.68(\mathrm{~m}, 1 \mathrm{H}), 4.88(\mathrm{~d}, J=13.2 \mathrm{~Hz}, 1 \mathrm{H}), 4.68(\mathrm{~d}, J$ $=13.2 \mathrm{~Hz}, 1 \mathrm{H}), 3.63$ (d, $J=10.0 \mathrm{~Hz}, 1 \mathrm{H}), 3.21$ (s, 3H), 2.88 (brs, 1H), 2.78 (brs, 1H); ${ }^{13} \mathrm{C}$ NMR (100 MHz, Acetone) $\delta 177.2,145.1,142.3,131.3,129.3,128.2,126.7,125.8,125.7,124.5$ (q, $J=$ $279.4 \mathrm{~Hz}), 123.1,112.7,80.0(\mathrm{q}, J=5.0 \mathrm{~Hz}), 64.8,62.1,36.5,26.4 ;{ }^{19} \mathrm{~F}$ NMR (376 MHz, Chloroform- $d$ ) $\delta$-80.25; IR (neat): $v$ 3322, 1703, 1609, 1375, 1177, 1164, 1034, 812, $771 \mathrm{~cm}^{-1}$; HRMS (ESI) Calcd. for $\mathrm{C}_{21} \mathrm{H}_{19} \mathrm{BrF}_{3} \mathrm{~N}_{2} \mathrm{O}_{2}[\mathrm{M}+\mathrm{H}]^{+}$: 467.0577, found: 467.0573 .

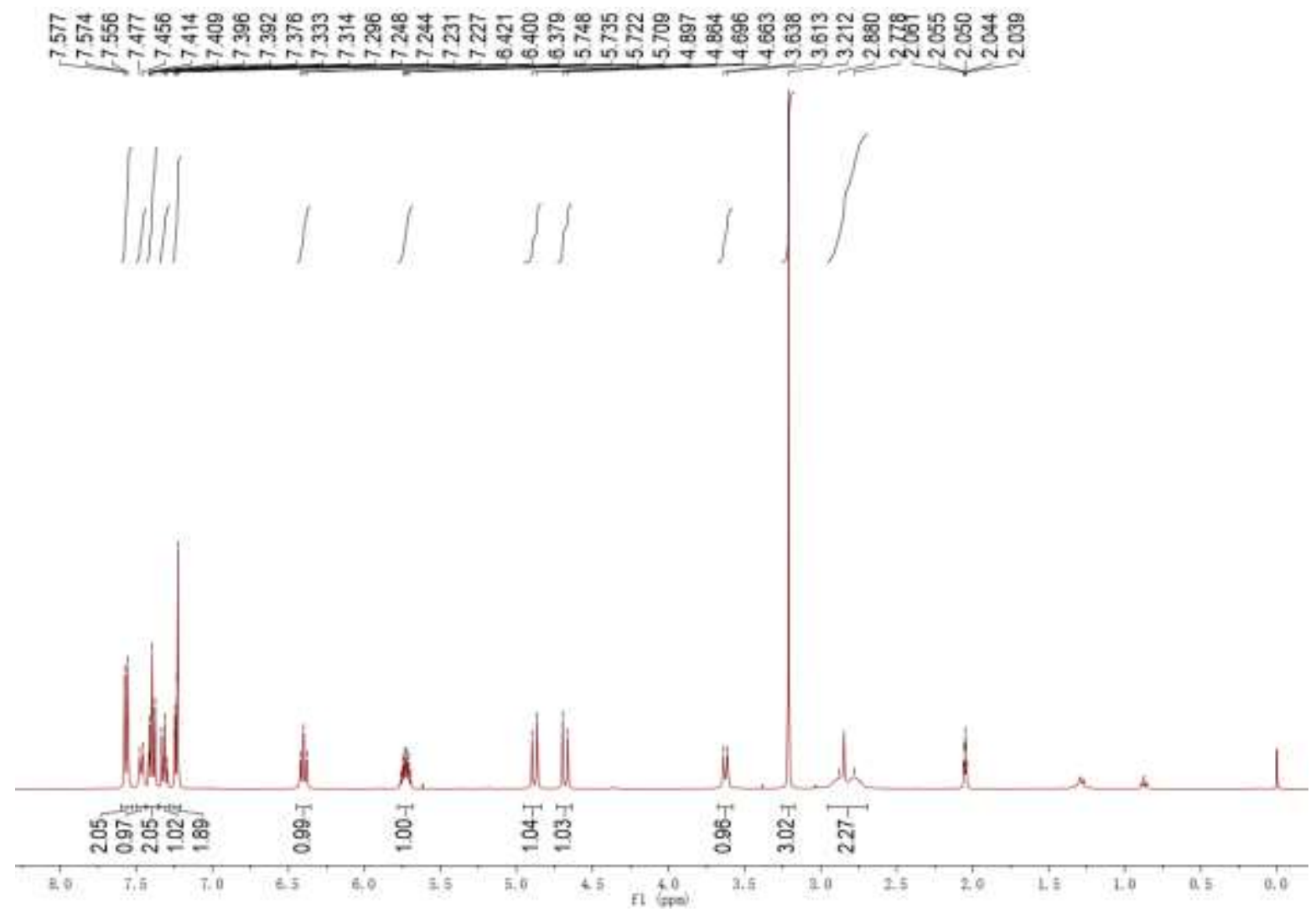



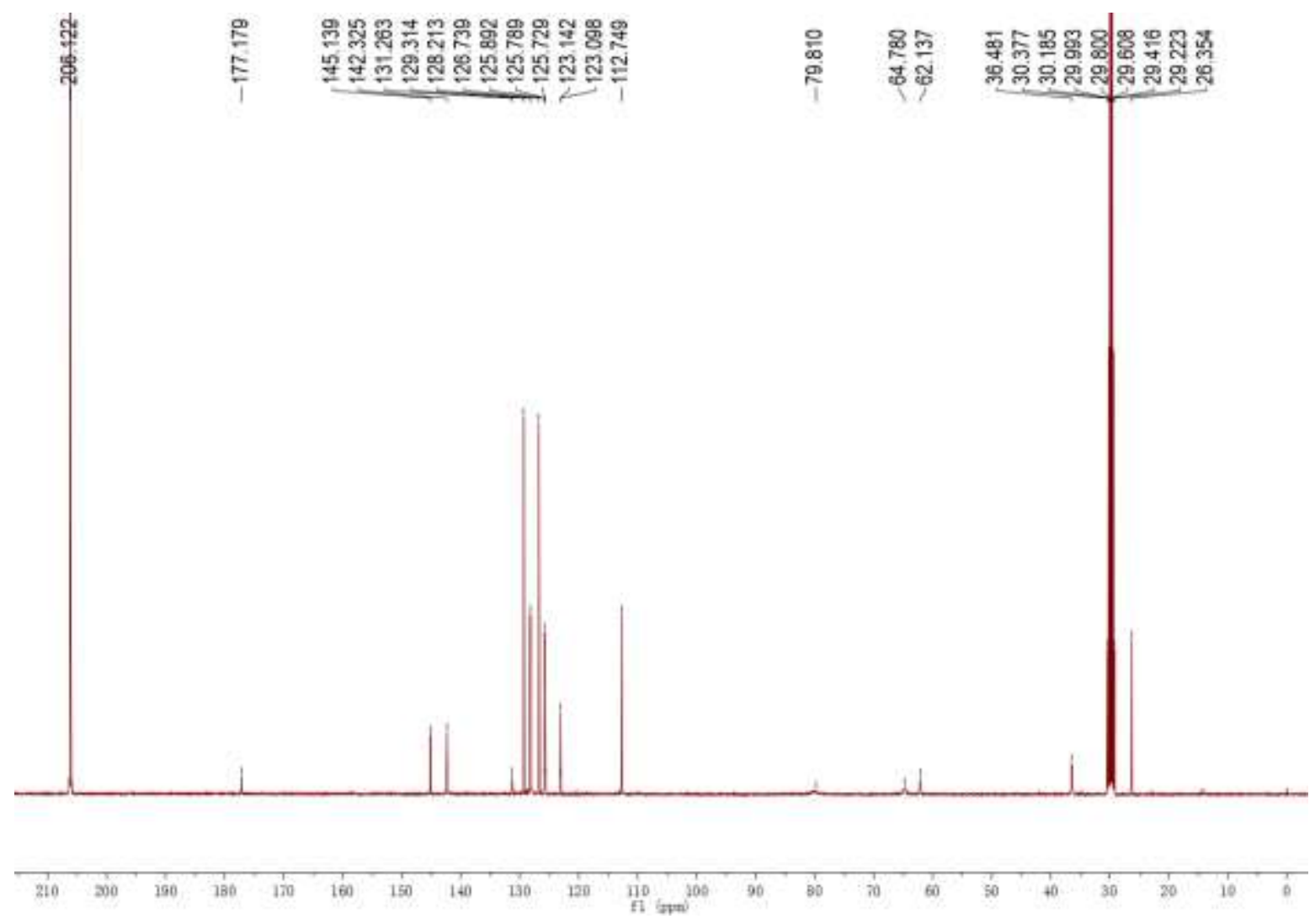

ํํํ

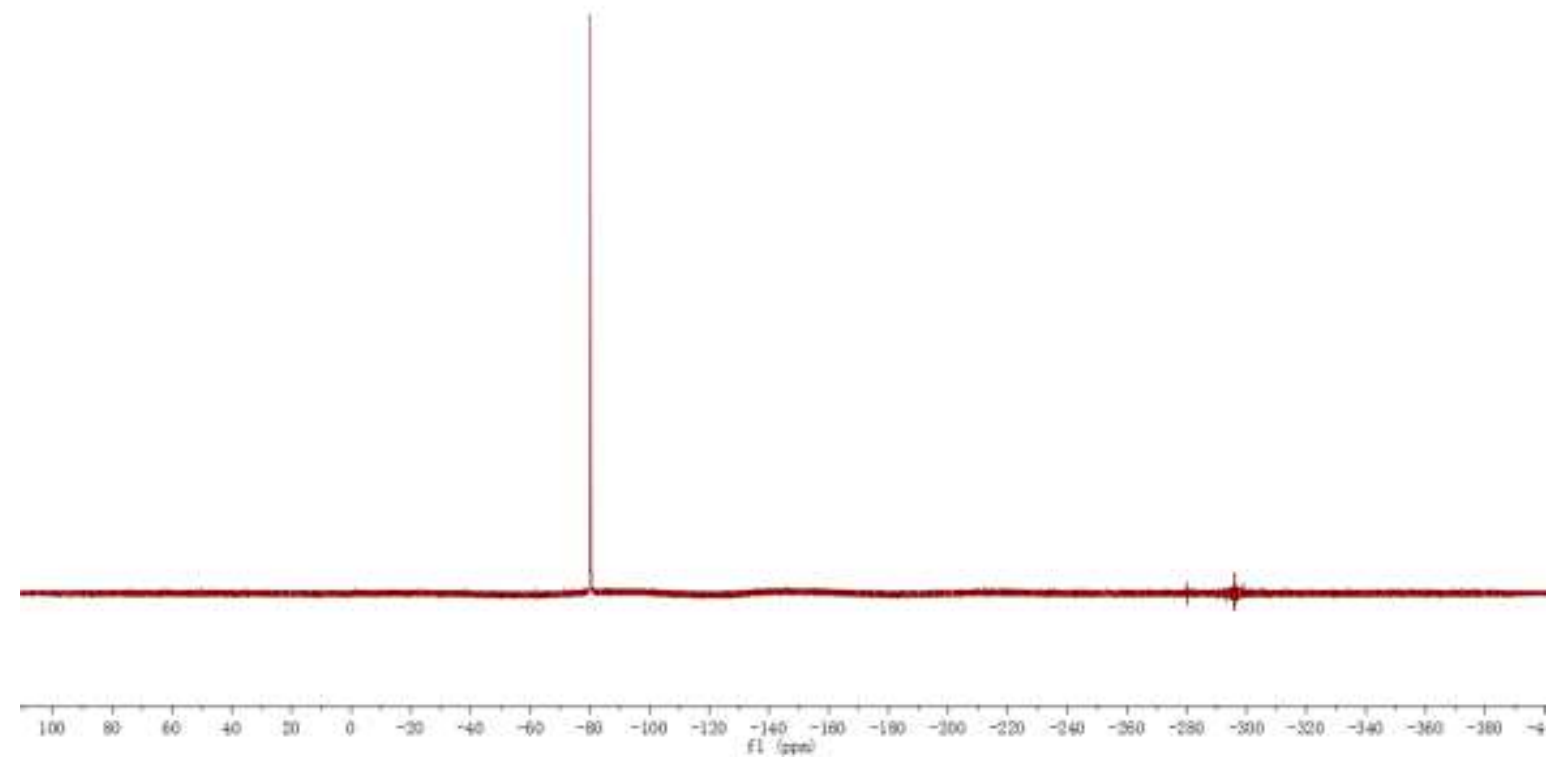




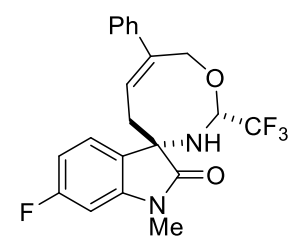

Compound 4ga: Yield: $58 \mathrm{mg}$, 72\%; A white solid; Mp: 185-187 ${ }^{\circ} \mathrm{C}$; ${ }^{1} \mathrm{H}$ NMR (400 MHz, Chloroform- $d$ ) $\delta$ 7.55-7.47 (m, 2H), 7.44-7.36 (m, 2H), 7.36-7.29 (m, 1H), $7.05(\mathrm{td}, J=8.8,2.4 \mathrm{~Hz}$, $1 \mathrm{H}), 6.93(\mathrm{dd}, J=5.2,2.4 \mathrm{~Hz}, 1 \mathrm{H}), 6.86-6.76(\mathrm{~m}, 1 \mathrm{H}), 6.28(\mathrm{t}, J=9.2 \mathrm{~Hz}, 1 \mathrm{H}), 5.75-5.62(\mathrm{~m}, 1 \mathrm{H})$, $4.80(\mathrm{~d}, J=13.6 \mathrm{~Hz}, 1 \mathrm{H}), 4.64$ (d, $J=13.6 \mathrm{~Hz}, 1 \mathrm{H}), 3.21$ (s, 3H), 3.05 (brs, 1H), 2.53 (brs, 1H), $2.43(\mathrm{~d}, J=10.0 \mathrm{~Hz}, 1 \mathrm{H}) ;{ }^{13} \mathrm{C} \mathrm{NMR}\left(100 \mathrm{MHz}, \mathrm{CDCl}_{3}\right) \delta 176.7,159.1(\mathrm{~d}, J=24.1 \mathrm{~Hz}), 141.0$, 138.51, 138.49, 132.0 (d, $J=7.4 \mathrm{~Hz}), 128.6,127.8,125.9,125.4,122.9(\mathrm{q}, J=280.0 \mathrm{~Hz}), 115.7(\mathrm{~d}$, $J=23.2 \mathrm{~Hz}), 110.9(\mathrm{~d}, J=24.9 \mathrm{~Hz}), 109.4(\mathrm{~d}, J=8.0 \mathrm{~Hz}), 81.0(\mathrm{q}, J=5.0 \mathrm{~Hz}), 66.2,62.1,35.3$, 26.3; ${ }^{19}$ F NMR (376 MHz, Chloroform- $d$ ) $\delta$-80.27, -119.25; IR (neat): v 3358, 2932, 1701, 1498, 1274, 1181, 1167, $775 \mathrm{~cm}^{-1}$; HRMS (ESI) Calcd. for $\mathrm{C}_{21} \mathrm{H}_{19} \mathrm{~F}_{4} \mathrm{~N}_{2} \mathrm{O}_{2}[\mathrm{M}+\mathrm{H}]^{+}:$407.1377, found: 407.1381.

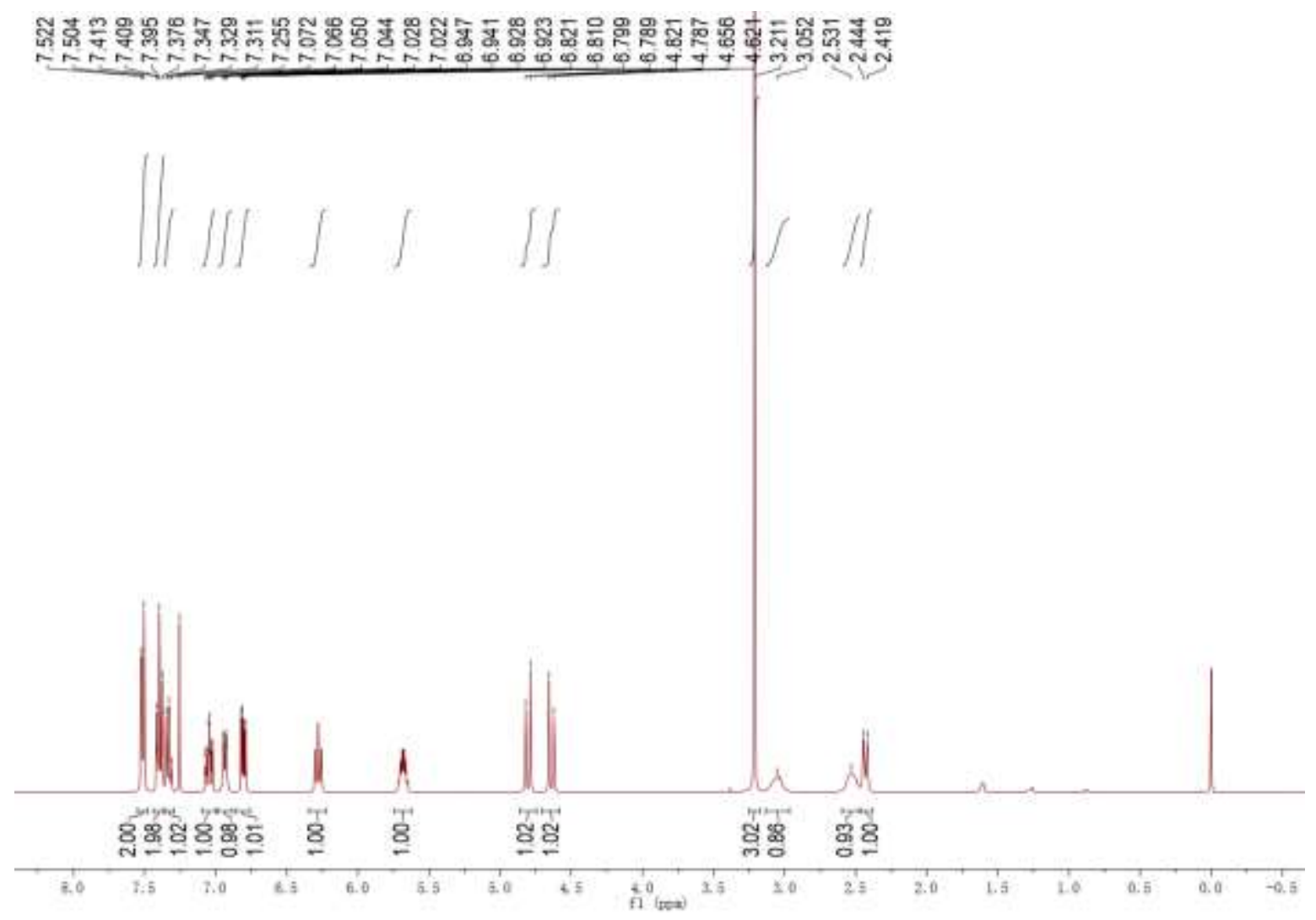



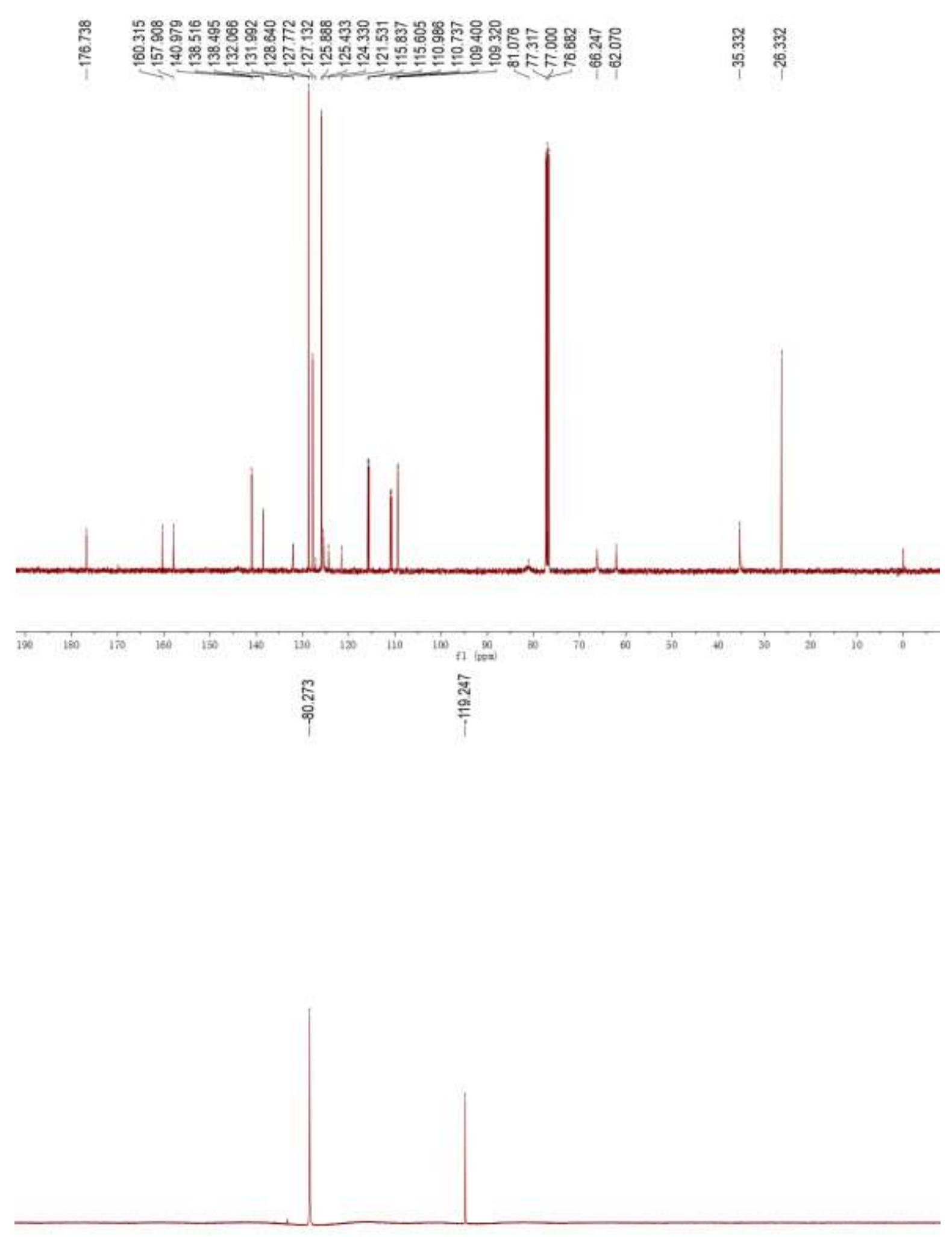

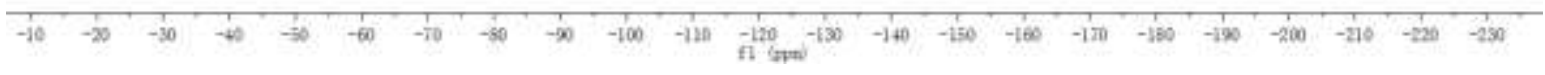

S23 


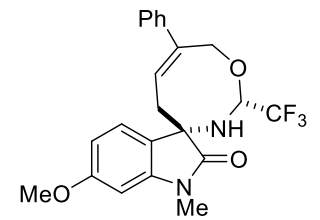

Compound 4ha: Yield: $67 \mathrm{mg}$, 80\%; A white solid; Mp: 173-175 ${ }^{\circ} \mathrm{C}$; ${ }^{1} \mathrm{H}$ NMR (400 MHz, Chloroform- $d$ ) $\delta 7.58-7.46(\mathrm{~m}, 2 \mathrm{H}), 7.45-7.28(\mathrm{~m}, 3 \mathrm{H}), 7.07(\mathrm{~d}, J=8.0 \mathrm{~Hz}, 1 \mathrm{H}), 6.51(\mathrm{~d}, J=8.0$ $\mathrm{Hz}, 1 \mathrm{H}), 6.44(\mathrm{~s}, 1 \mathrm{H}), 6.30(\mathrm{t}, J=8.4 \mathrm{~Hz}, 1 \mathrm{H}), 5.73-5.56(\mathrm{~m}, 1 \mathrm{H}), 4.80(\mathrm{~d}, J=13.6 \mathrm{~Hz}, 1 \mathrm{H}), 4.63(\mathrm{~d}$, $J=13.6 \mathrm{~Hz}, 1 \mathrm{H}), 3.82(\mathrm{~s}, 3 \mathrm{H}), 3.19$ (s, 3H), 3.02 (brs, 1H), 2.52 (brs, 1H), 2.42 (d, $J=10.4 \mathrm{~Hz}, 1 \mathrm{H})$; ${ }^{13} \mathrm{C}$ NMR $\left(100 \mathrm{MHz}, \mathrm{CDCl}_{3}\right) \delta 177.6,161.2,144.0,141.3,128.6,127.6,126.4,125.9,123.2$, $123.05,123.03(\mathrm{~d}, J=280.0 \mathrm{~Hz}), 106.3,96.8,81.4(\mathrm{q}, J=5.0 \mathrm{~Hz}), 66.1,61.5,55.6,35.6,26.2 ;{ }^{19} \mathrm{~F}$ NMR (376 MHz, Chloroform-d) $\delta$-80.25; IR (neat): v 3345, 2924, 1697, 1616, 1379, 1154, 1089, $1076 \mathrm{~cm}^{-1}$; HRMS (ESI) Calcd. for $\mathrm{C}_{22} \mathrm{H}_{22} \mathrm{~F}_{3} \mathrm{~N}_{2} \mathrm{O}_{3}[\mathrm{M}+\mathrm{H}]^{+}:$419.1577, found: 419.1581 .

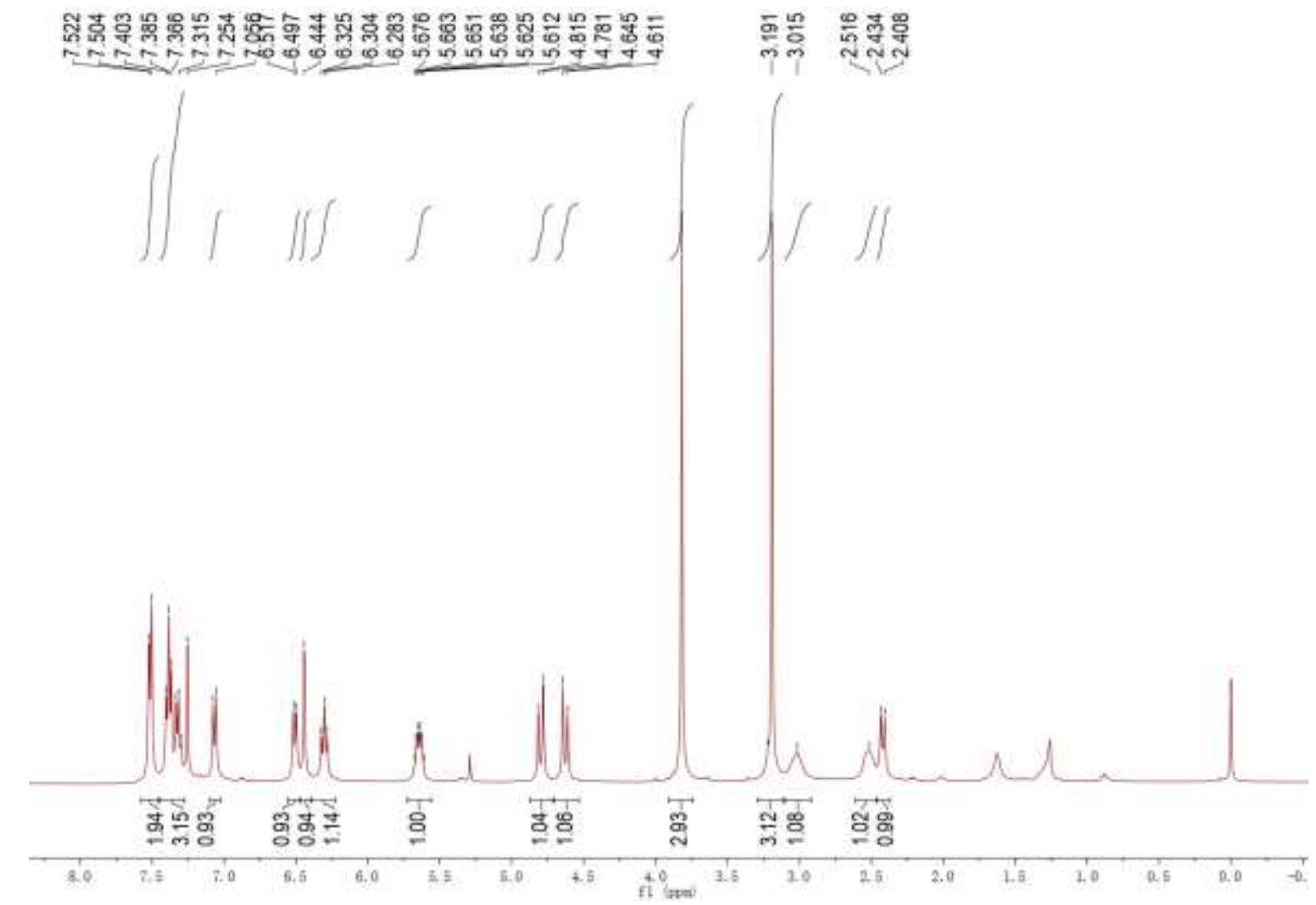




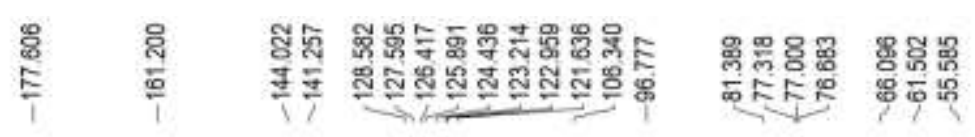

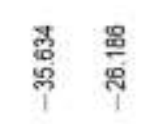
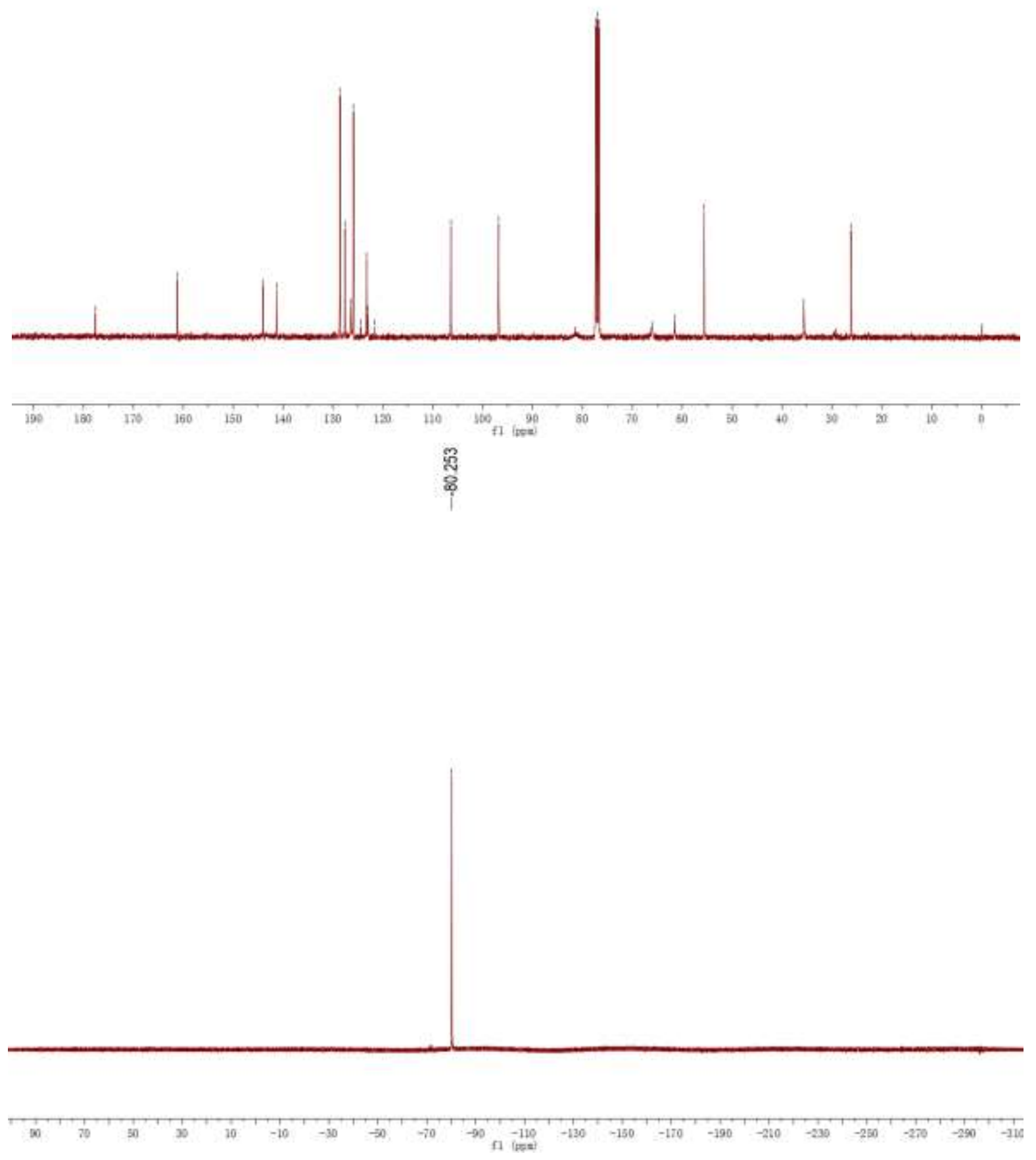

$\mathrm{S} 25$ 


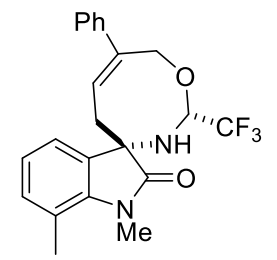

Compound 4ia: Yield: $65 \mathrm{mg}$, 81\%; A white solid; Mp: 153-155 ${ }^{\circ} \mathrm{C}$; ${ }^{1} \mathrm{H}$ NMR (400 MHz, Chloroform- $d$ ) $\delta$ 7.58-7.46 (m, 2H), 7.44-7.26 (m, 3H), $7.06(\mathrm{~d}, J=7.6 \mathrm{~Hz}, 1 \mathrm{H}), 7.00(\mathrm{~d}, J=6.8$ $\mathrm{Hz}, 1 \mathrm{H}), 6.91(\mathrm{t}, J=7.6 \mathrm{~Hz}, 1 \mathrm{H}), 6.30$ (t, $J=7.6 \mathrm{~Hz}, 1 \mathrm{H}), 5.75-5.59$ (m, 1H), 4.80 (d, $J=13.6 \mathrm{~Hz}$, 1H), 4.63 (d, $J=13.6 \mathrm{~Hz}, 1 \mathrm{H}), 3.49$ (s, 3H), 2.96 (brs, 1H), 2.57 (brs, 4H), 2.41 (d, $J=10.0 \mathrm{~Hz}$, $1 \mathrm{H}) ;{ }^{13} \mathrm{C} \mathrm{NMR}\left(100 \mathrm{MHz}, \mathrm{CDCl}_{3}\right) \delta 177.6,141.2,140.1,133.2,131.5,128.6,127.6,126.5,125.9$, $123.0(\mathrm{q}, J=279.8 \mathrm{~Hz}) 122.8,120.4,120.2,81.0(\mathrm{q}, J=5.0 \mathrm{~Hz}), 65.9,61.0,35.8,29.5,19.0 ;{ }^{19} \mathrm{~F}$ NMR (376 MHz, Chloroform-d) $\delta$-80.28; IR (neat): v 3311, 2921, 1802, 1705, 1462, 1192, 1171, $787 \mathrm{~cm}^{-1}$; HRMS (ESI) Calcd. for $\mathrm{C}_{22} \mathrm{H}_{22} \mathrm{~F}_{3} \mathrm{~N}_{2} \mathrm{O}_{2}[\mathrm{M}+\mathrm{H}]^{+}: 403.1628$, found: 403.1632 .

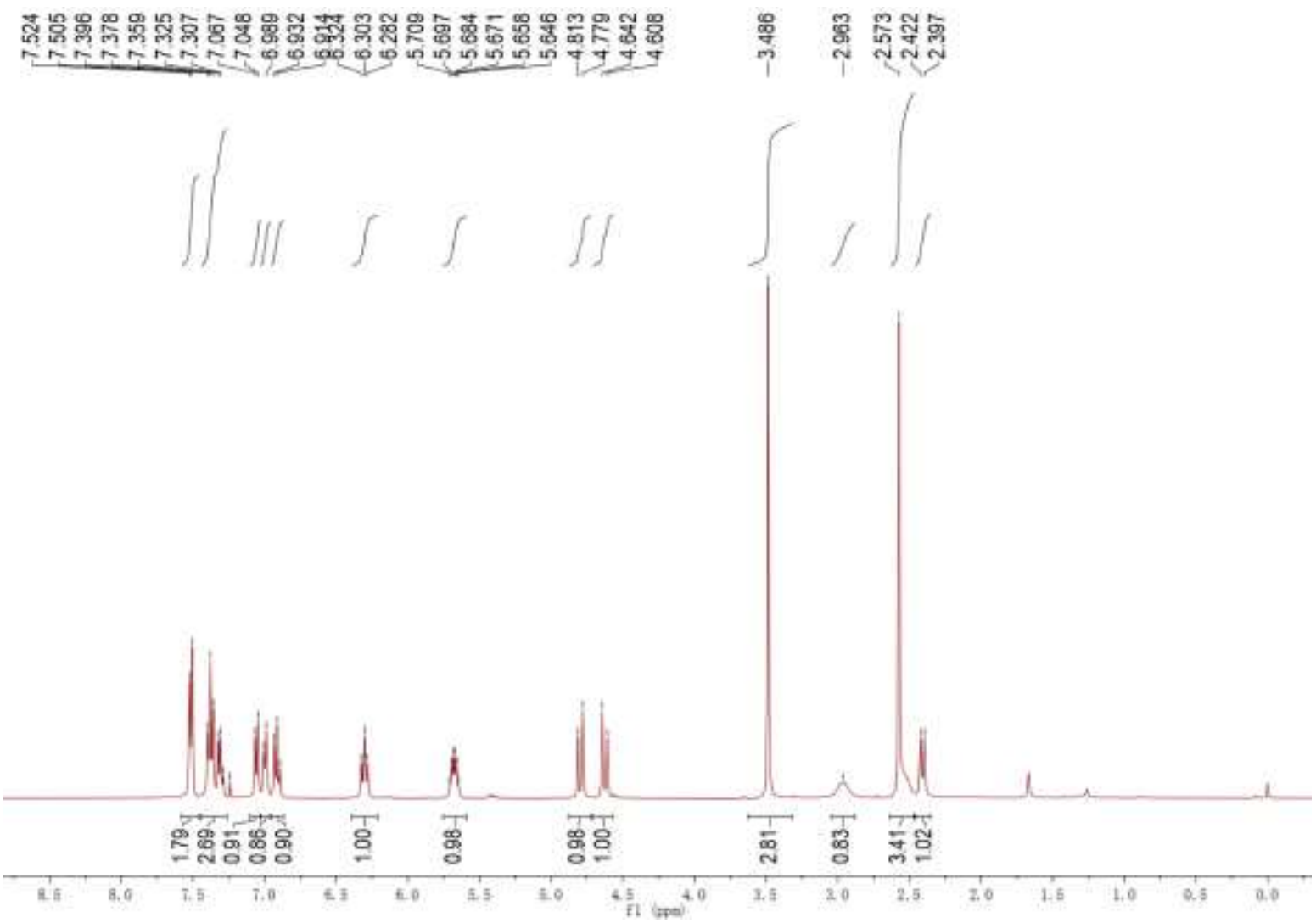



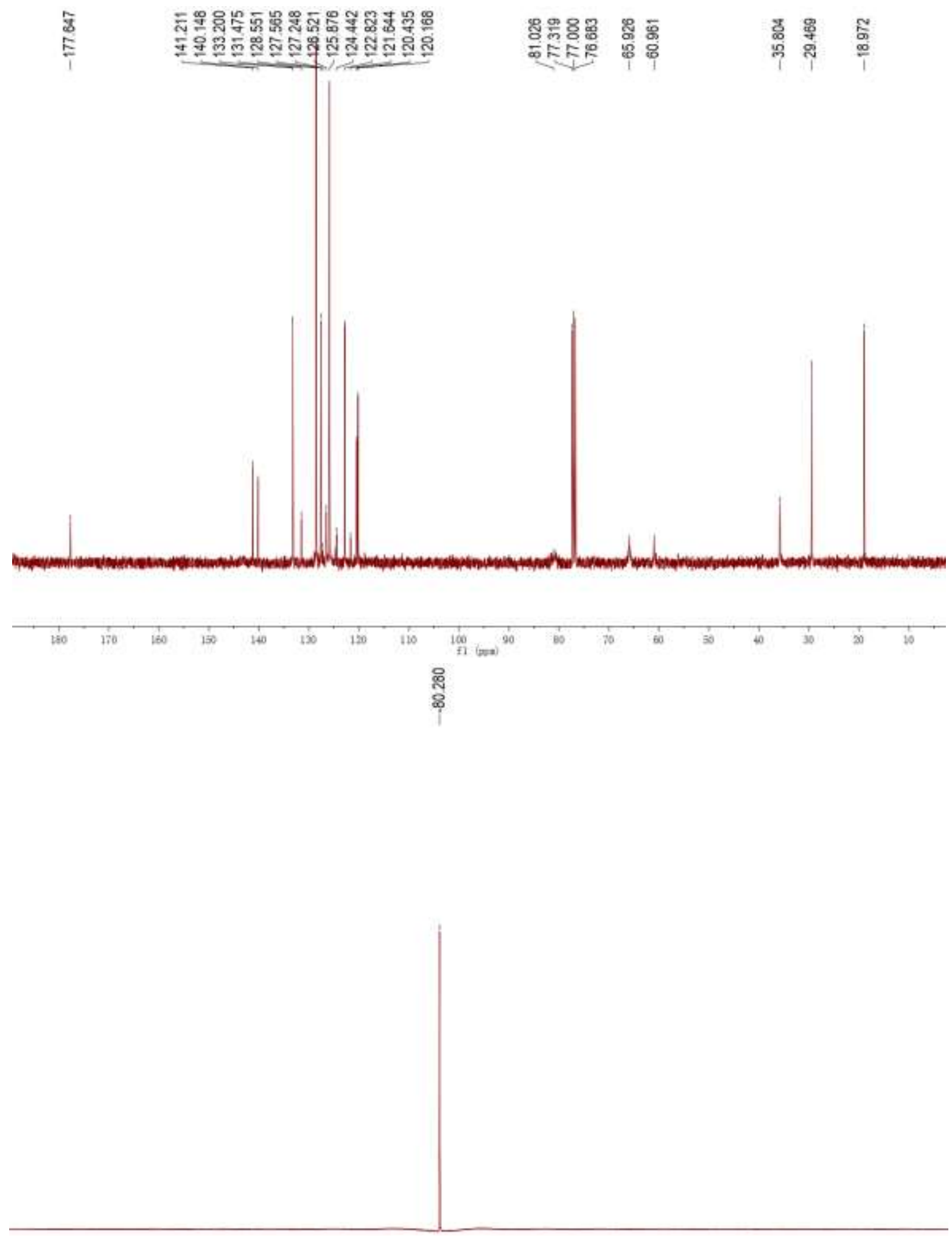

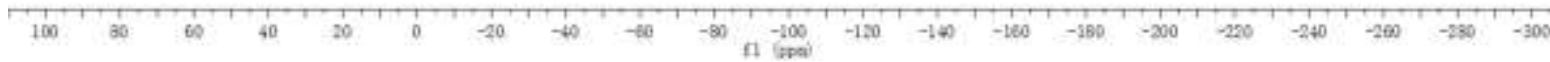




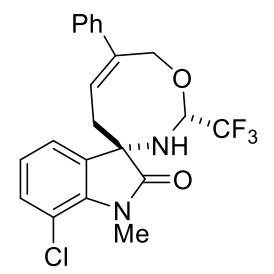

Compound 4ja: Yield: $68 \mathrm{mg}$, 81\%; A white solid; Mp: 175-176 ${ }^{\circ} \mathrm{C}$; ${ }^{1} \mathrm{H}$ NMR (400 MHz, Chloroform-d) $\delta 7.56-7.46(\mathrm{~m}, 2 \mathrm{H}), 7.39(\mathrm{t}, J=7.6 \mathrm{~Hz}, 2 \mathrm{H}), 7.35-7.28(\mathrm{~m}, 1 \mathrm{H}), 7.26(\mathrm{~d}, J=7.2 \mathrm{~Hz}$, 1H), $7.05(\mathrm{~d}, J=7.2 \mathrm{~Hz}, 1 \mathrm{H}), 6.94(\mathrm{t}, J=8.0 \mathrm{~Hz}, 1 \mathrm{H}), 6.26$ (t, $J=8.4 \mathrm{~Hz}, 1 \mathrm{H}), 5.71-5.59(\mathrm{~m}, 1 \mathrm{H})$, $4.80(\mathrm{~d}, J=13.6 \mathrm{~Hz}, 1 \mathrm{H}), 4.63(\mathrm{~d}, J=13.6 \mathrm{~Hz}, 1 \mathrm{H}), 3.58(\mathrm{~s}, 3 \mathrm{H}), 2.99$ (brs, 1H), 2.55 (brs, 1H), $2.41(\mathrm{~d}, J=9.6 \mathrm{~Hz}, 1 \mathrm{H}) ;{ }^{13} \mathrm{C} \mathrm{NMR}\left(100 \mathrm{MHz}, \mathrm{CDCl}_{3}\right) \delta 177.2,141.0,138.5,133.4,131.8,128.6$, $127.7,125.9,125.8,123.7,122.9(\mathrm{q}, J=280.1 \mathrm{~Hz}), 120.9,116.1,81.0(\mathrm{q}, J=6.0 \mathrm{~Hz}), 66.2,61.3$, 35.6, 29.5; ${ }^{19} \mathrm{~F}$ NMR (376 MHz, Chloroform- $d$ ) $\delta$-80.23; IR (neat): $v 3327,1697,1611,1471,1173$, 1165, 1154, $777 \mathrm{~cm}^{-1}$; HRMS (ESI) Calcd. for $\mathrm{C}_{21} \mathrm{H}_{19} \mathrm{ClF}_{3} \mathrm{~N}_{2} \mathrm{O}_{2}[\mathrm{M}+\mathrm{H}]^{+}$: 423.1082, found: 423.1080.

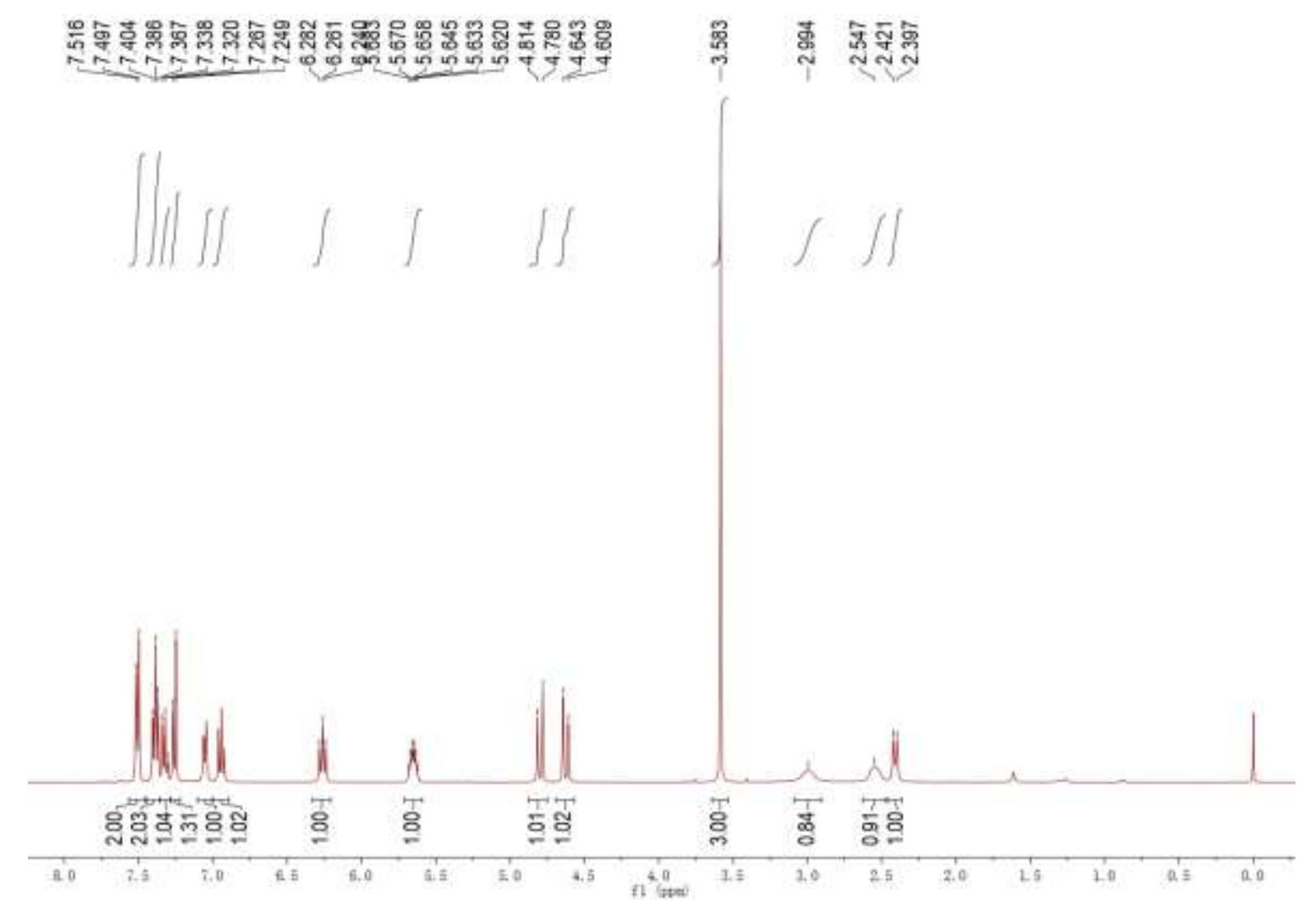




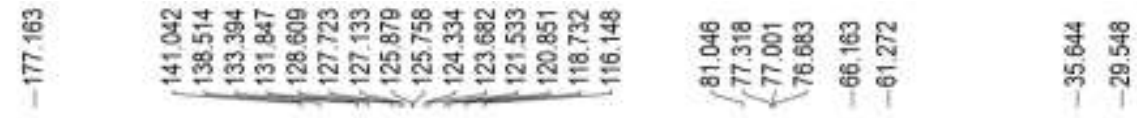
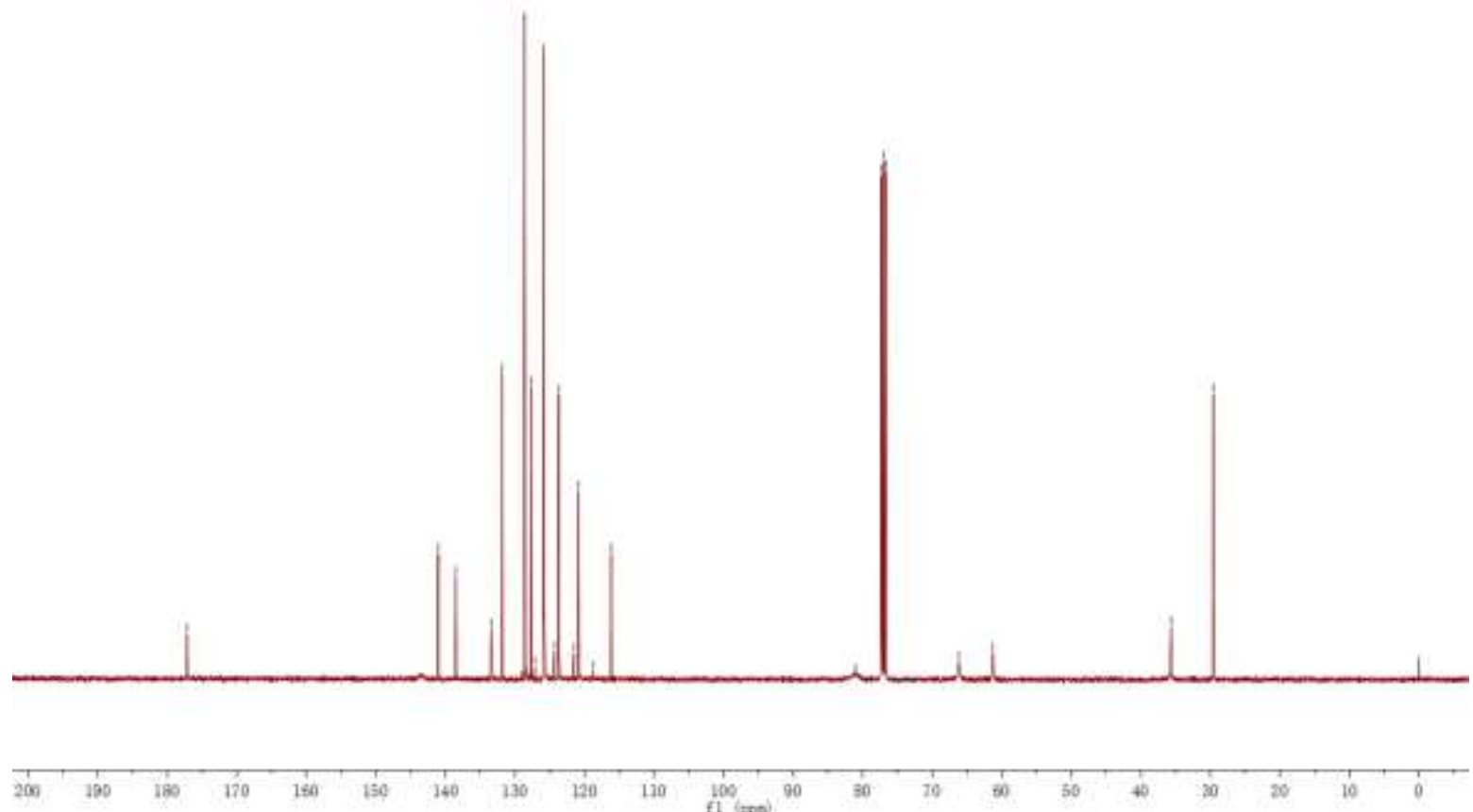

m

i

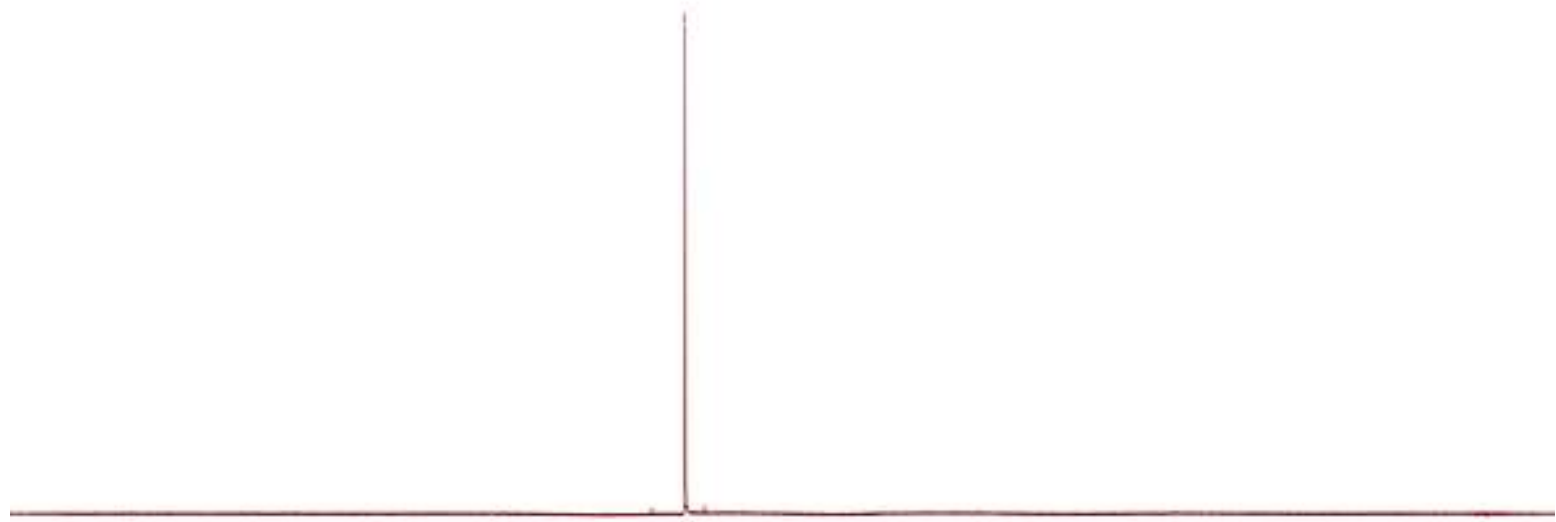

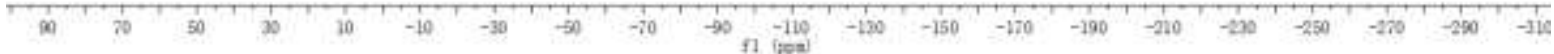


<smiles></smiles>

Compound 4ka: Yield: $74 \mathrm{mg}$, 80\%; A white solid; Mp: 175-177 ${ }^{\circ} \mathrm{C}$; ${ }^{1} \mathrm{H}$ NMR (400 MHz, Chloroform- $d$ ) $\delta$ 7.59-7.49 (m, 2H), 7.45-7.36 (m, 2H), 7.35-7.13 (m, 8H), $6.99(\mathrm{t}, J=7.2 \mathrm{~Hz}, 1 \mathrm{H})$, $6.74(\mathrm{~d}, J=8.0 \mathrm{~Hz}, 1 \mathrm{H}), 6.37(\mathrm{t}, J=8.4 \mathrm{~Hz}, 1 \mathrm{H}), 5.79-5.68(\mathrm{~m}, 1 \mathrm{H}), 5.00(\mathrm{~d}, J=15.6 \mathrm{~Hz}, 1 \mathrm{H})$, 4.86-4.76 (m, 2H), 4.66 (d, $J=13.6 \mathrm{~Hz}, 1 \mathrm{H}), 3.09$ (brs, 1H), 2.63 (brs, 1H), 2.49 (d, $J=10.4 \mathrm{~Hz}$, 1H); ${ }^{13} \mathrm{C}$ NMR $\left(100 \mathrm{MHz}, \mathrm{CDCl}_{3}\right) \delta 177.1,141.6,141.2,135.4,130.9,129.5,128.8,128.6,127.7$, 127.6, 127.1, 126.4, 125.9, 123.1 (q, $J=280.0 \mathrm{~Hz}), 122.9,122.4,109.7,81.0$ (q, $J=5.0 \mathrm{~Hz}), 65.9$, 61.8, 43.4, 35.4; ${ }^{19}$ F NMR (376 MHz, Chloroform- $d$ ) $\delta$-80.39; IR (neat): v 3324, 1698, 1614, 1489, 1173, 1153, 766, $697 \mathrm{~cm}^{-1}$; HRMS (ESI) Calcd. for $\mathrm{C}_{27} \mathrm{H}_{24} \mathrm{~F}_{3} \mathrm{~N}_{2} \mathrm{O}_{2}[\mathrm{M}+\mathrm{H}]^{+}:$465.1784, found: 465.1779 .

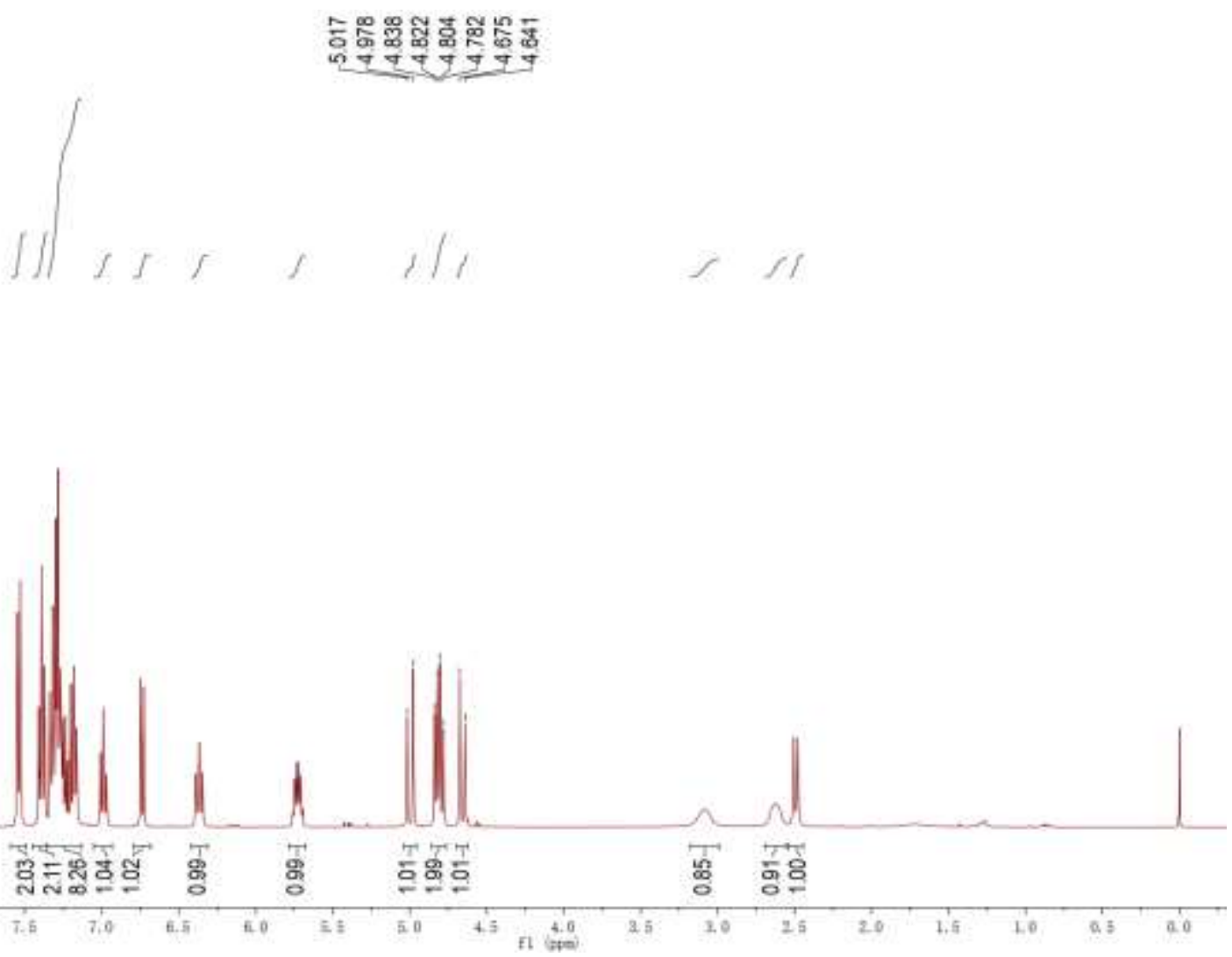



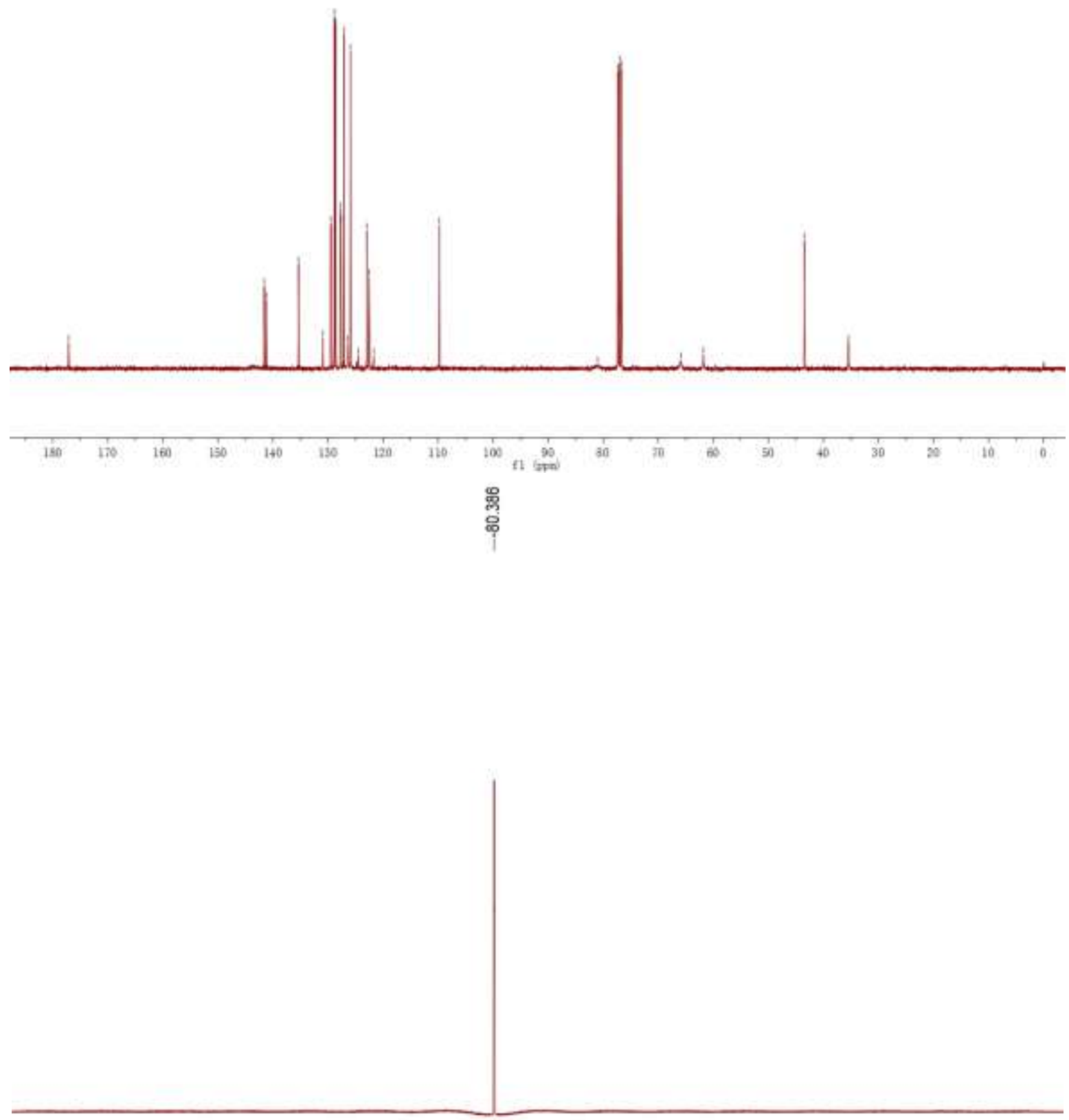

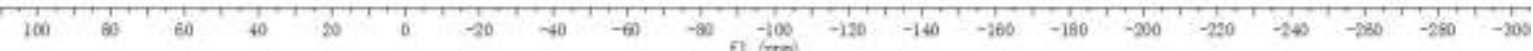


<smiles>Cc1cc(C)c2c(c1)C1(CC=C(c3ccccc3)CO1)NC(=O)[C@H]2Br</smiles>

Compound 4la: Yield: $72 \mathrm{mg}$, 73\%; A white solid; Mp: 163-165 ${ }^{\circ} \mathrm{C}$; ${ }^{1} \mathrm{H}$ NMR (400 MHz, Chloroform- $d$ ) $\delta 7.58-7.50(\mathrm{~m}, 2 \mathrm{H}), 7.38(\mathrm{t}, J=7.6 \mathrm{~Hz}, 2 \mathrm{H}), 7.35-7.26(\mathrm{~m}, 3 \mathrm{H}), 7.26-7.19(\mathrm{~m}, 1 \mathrm{H})$, $7.15(\mathrm{~d}, J=7.6 \mathrm{~Hz}, 2 \mathrm{H}), 6.89(\mathrm{~s}, 1 \mathrm{H}), 6.79(\mathrm{~s}, 1 \mathrm{H}), 6.40(\mathrm{t}, J=8.4 \mathrm{~Hz}, 1 \mathrm{H}), 5.78-5.65(\mathrm{~m}, 1 \mathrm{H}), 5.26$ $(\mathrm{d}, J=16.8 \mathrm{~Hz}, 1 \mathrm{H}), 5.03(\mathrm{~d}, J=16.8 \mathrm{~Hz}, 1 \mathrm{H}), 4.82(\mathrm{~d}, J=13.2 \mathrm{~Hz}, 1 \mathrm{H}), 4.66(\mathrm{~d}, J=13.2 \mathrm{~Hz}, 1 \mathrm{H})$, 2.99 (brs, 1H), 2.69 (brs, 1H), 2.48 (d, $J=10.4 \mathrm{~Hz}, 1 \mathrm{H}), 2.24$ (s, 3H), $2.23(\mathrm{~s}, 3 \mathrm{H}) ;{ }^{13} \mathrm{C}$ NMR (100 $\left.\mathrm{MHz} \mathrm{CDCl}_{3}\right) \delta 177.9,141.2,137.2,137.0,133.7,132.8,132.0,128.9,128.6,127.6,127.2,125.9$, 125.5, $123.2(\mathrm{q}, J=280.0 \mathrm{~Hz}), 120.9,120.3,80.7(\mathrm{q}, J=5.0 \mathrm{~Hz}), 65.7,60.9,44.6,36.0,20.7,18.6$; ${ }^{19}$ F NMR (376 MHz, Chloroform- $d$ ) $\delta$-80.30; IR (neat): v 3369, 2908, 1708, 1181, 1153, 1042, 819, $695 \mathrm{~cm}^{-1}$; HRMS (ESI) Calcd. for $\mathrm{C}_{29} \mathrm{H}_{28} \mathrm{~F}_{3} \mathrm{~N}_{2} \mathrm{O}_{2}[\mathrm{M}+\mathrm{H}]^{+}:$493.2097, found: 493.2092 .

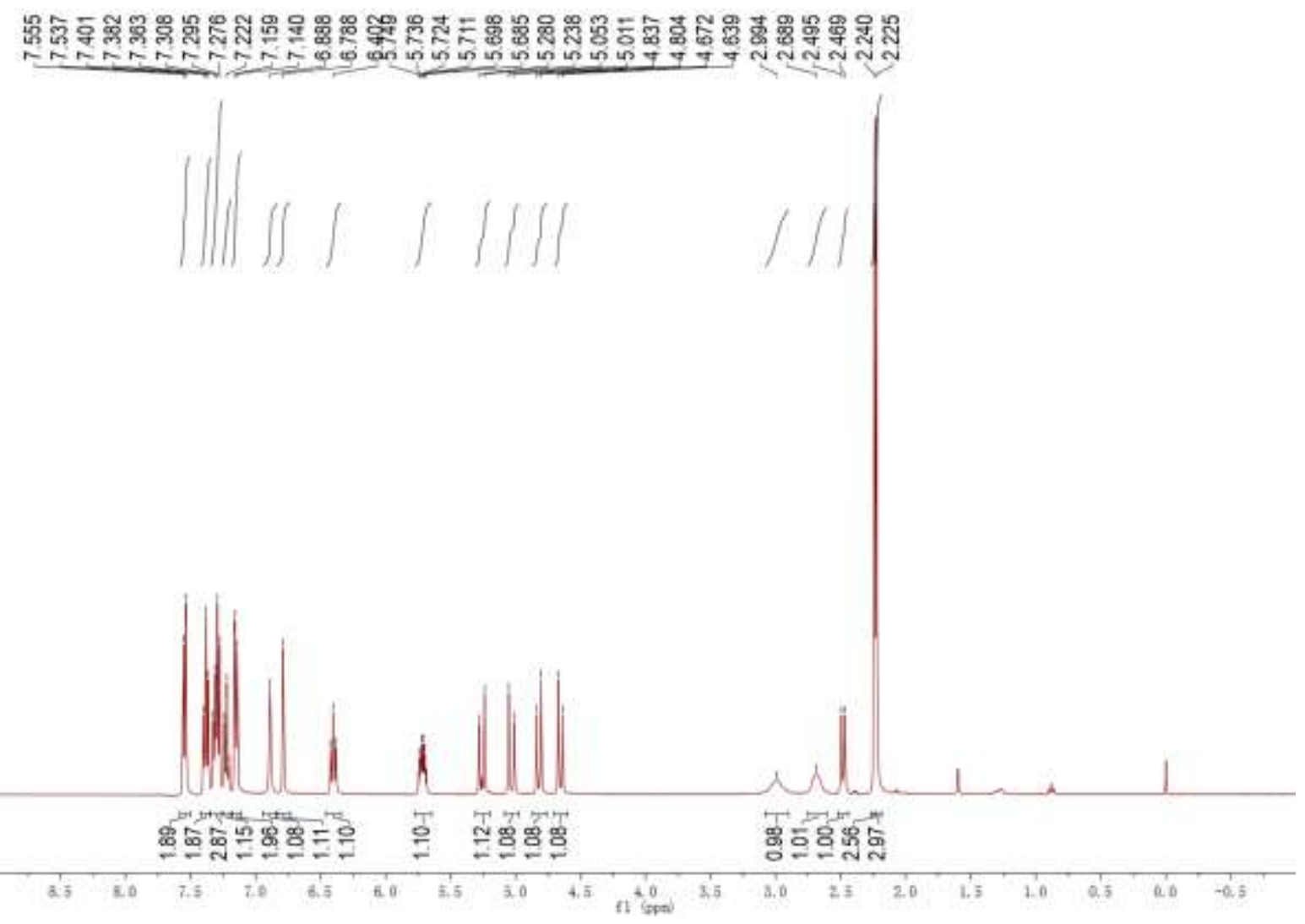




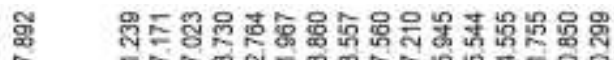

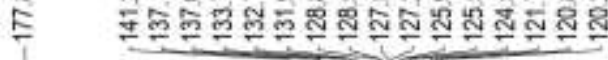

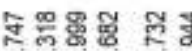

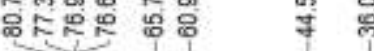

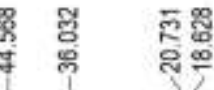
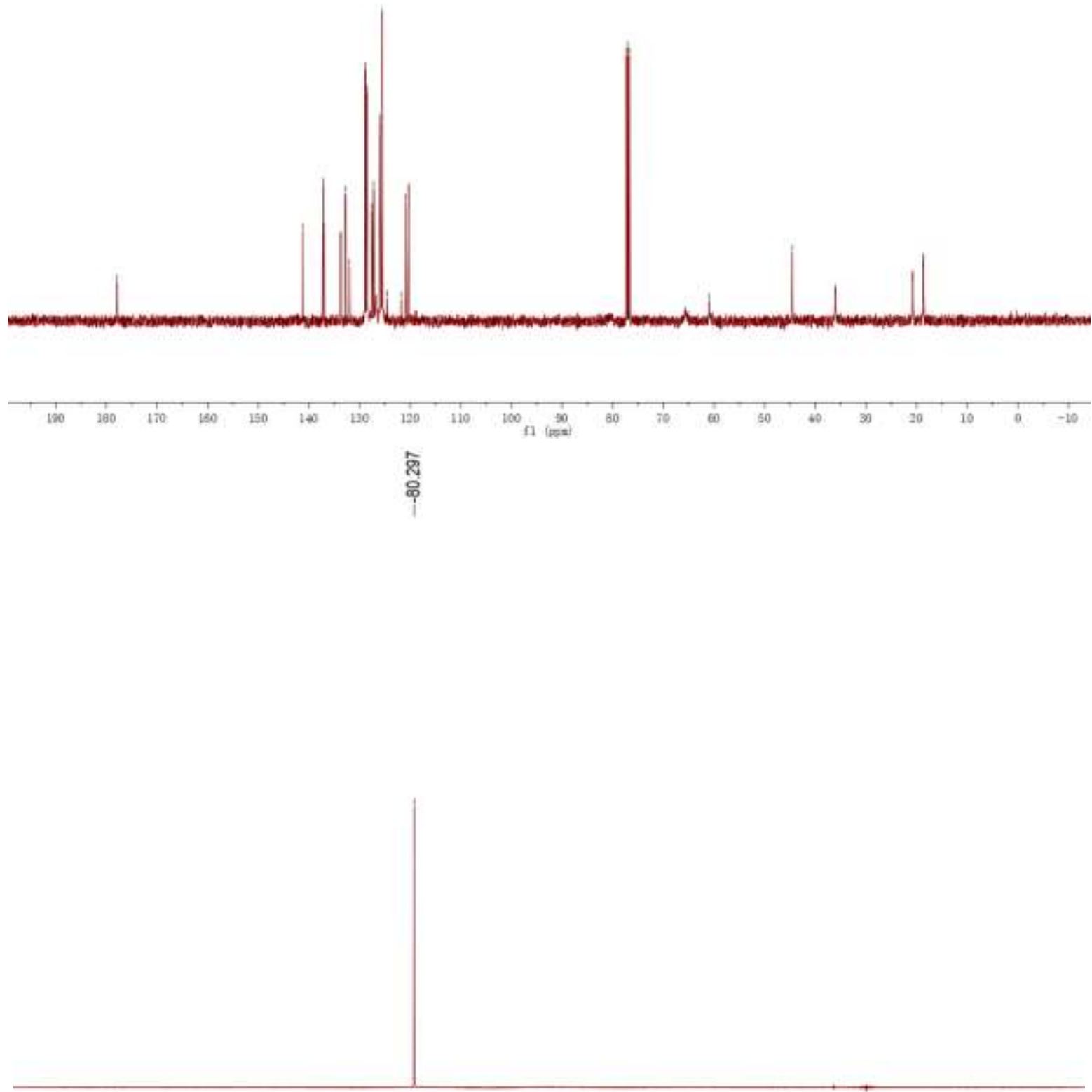

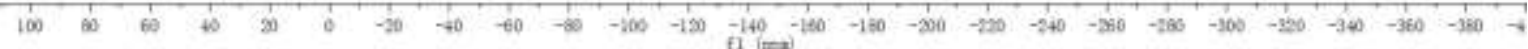


<smiles>COc1ccc2c(c1)C1(CC=C(c3ccccc3)COC(C(F)(F)F)N1)C(=O)N2</smiles>

Compound 4ma: Yield: $81 \mathrm{mg}$, 82\%; A white solid; Mp: 187-189 ${ }^{\circ} \mathrm{C}$; ${ }^{1} \mathrm{H}$ NMR (400 MHz, Chloroform- $d$ ) $\delta 7.59-7.48(\mathrm{~m}, 2 \mathrm{H}), 7.38(\mathrm{t}, J=8.0 \mathrm{~Hz}, 2 \mathrm{H}), 7.34-7.21(\mathrm{~m}, 5 \mathrm{H}), 6.80(\mathrm{~s}, 1 \mathrm{H}), 6.71$ $(\mathrm{dd}, J=6.0,2.4 \mathrm{~Hz}, 1 \mathrm{H}), 6.67-6.57(\mathrm{~m}, 1 \mathrm{H}), 6.37$ (t, $J=8.4 \mathrm{~Hz}, 1 \mathrm{H}), 5.81-5.67(\mathrm{~m}, 1 \mathrm{H}), 4.98(\mathrm{~d}, J$ $=15.6 \mathrm{~Hz}, 1 \mathrm{H}), 4.80-4.70(\mathrm{~m}, 2 \mathrm{H}), 4.65(\mathrm{~d}, J=13.2 \mathrm{~Hz}, 1 \mathrm{H}), 3.70(\mathrm{~s}, 3 \mathrm{H}), 3.05$ (brs, 1H), 2.63 (brs, 1H), $2.48(\mathrm{~d}, J=10.0 \mathrm{~Hz}, 1 \mathrm{H}) ;{ }^{13} \mathrm{C} \mathrm{NMR}\left(100 \mathrm{MHz}, \mathrm{CDCl}_{3}\right) \delta 176.7,156.1,141.1,135.4,134.8$, $132.1,128.8,128.6,127.7,127.6,127.0,125.9,123.1(\mathrm{q}, J=280.3 \mathrm{~Hz}), 112.9,110.5,110.1,81.0(\mathrm{q}$, $J=5.0 \mathrm{~Hz}), 65.7,62.0,55.7,43.5,35.5 ;{ }^{19} \mathrm{~F}$ NMR (376 MHz, Chloroform- $d$ ) $\delta$-80.27; IR (neat): $v$ 3309, 1702, 1493, 1170, 1063, 1044, 804, $712 \mathrm{~cm}^{-1}$; HRMS (ESI) Calcd. for $\mathrm{C}_{28} \mathrm{H}_{26} \mathrm{~F}_{3} \mathrm{~N}_{2} \mathrm{O}_{3}[\mathrm{M}+\mathrm{H}]^{+}$: 495.1890, found: 495.1892 .

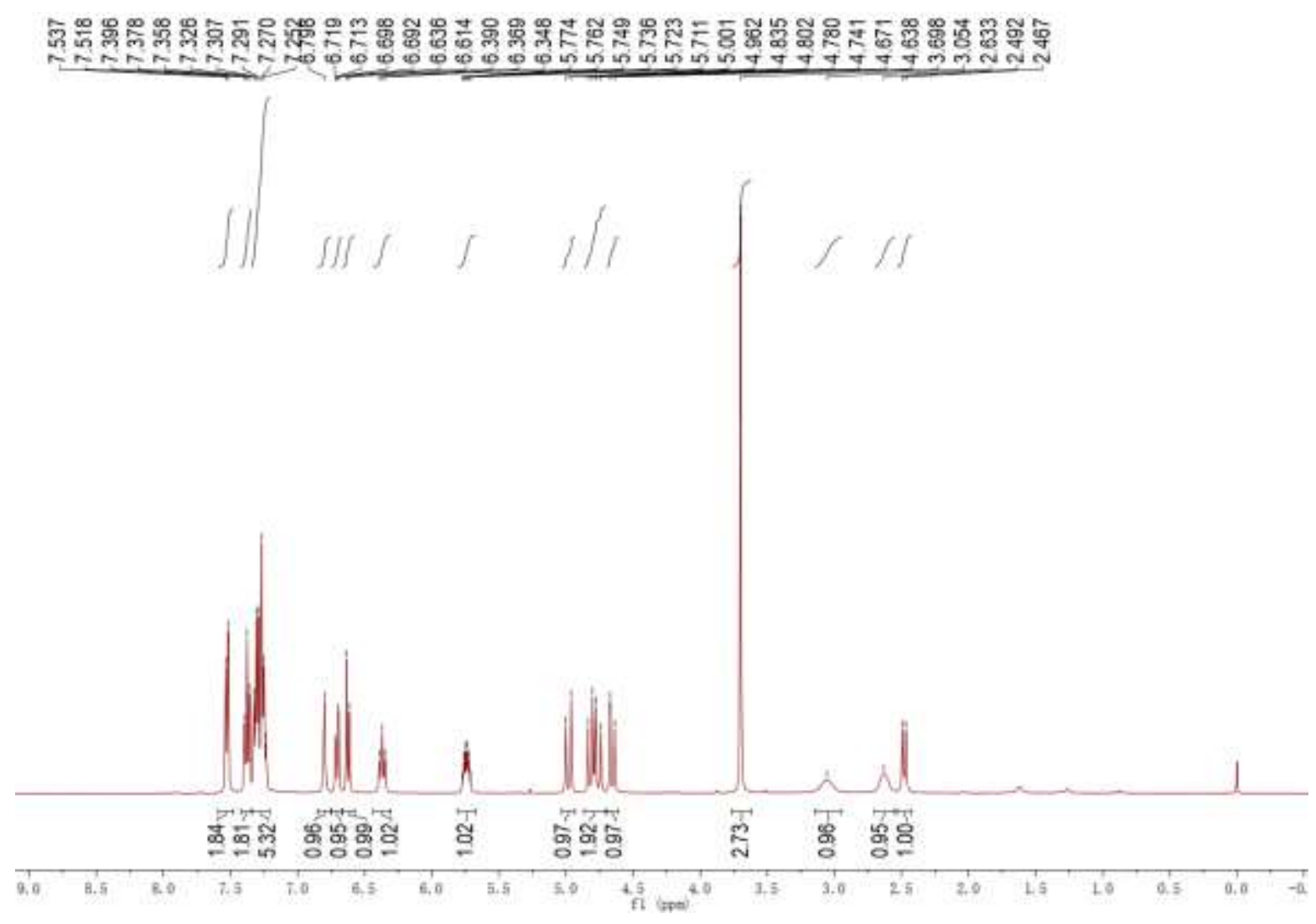




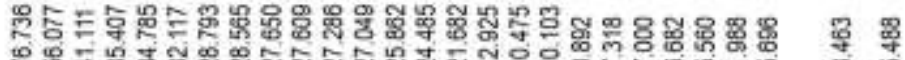

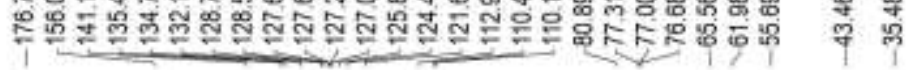
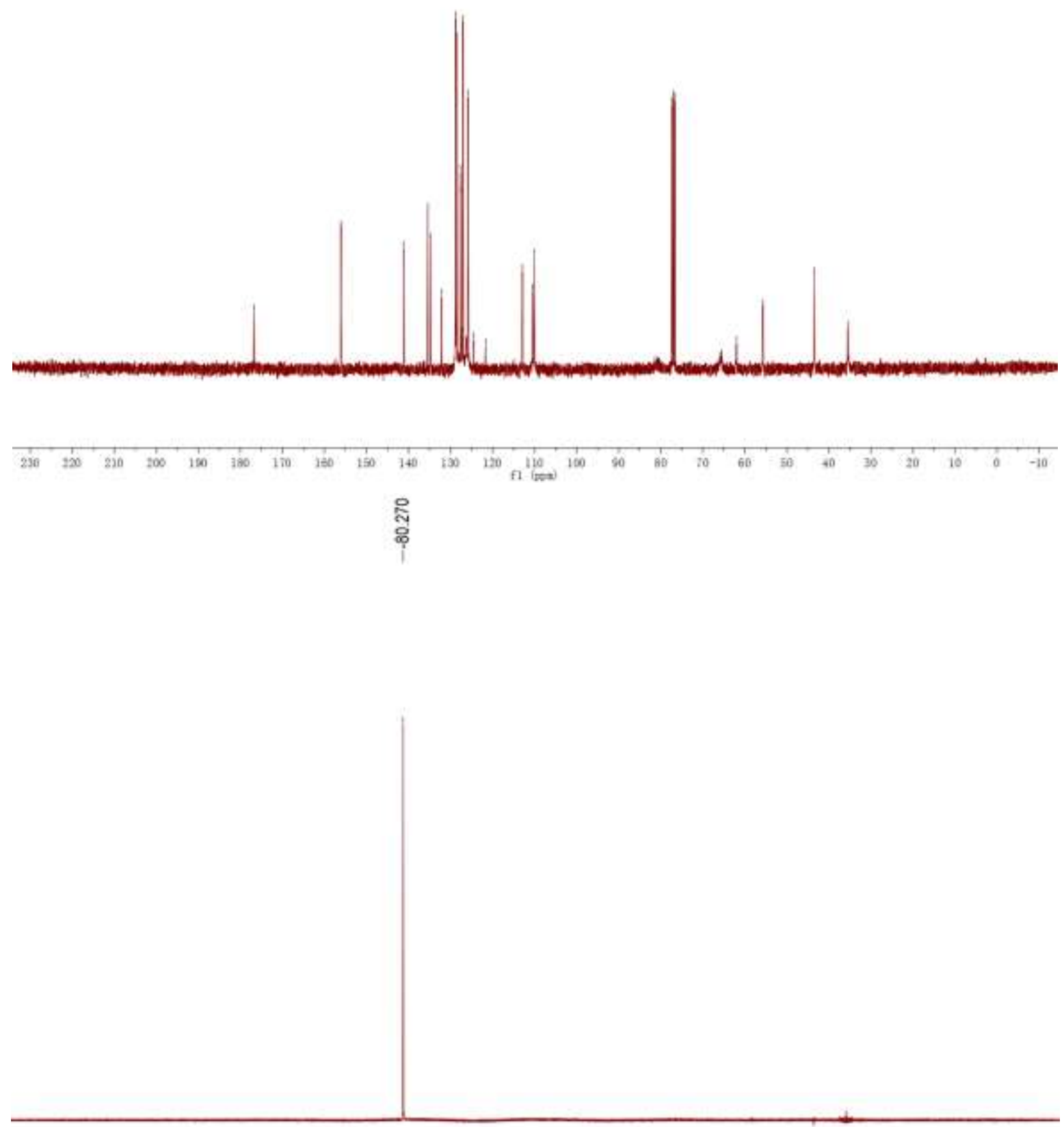

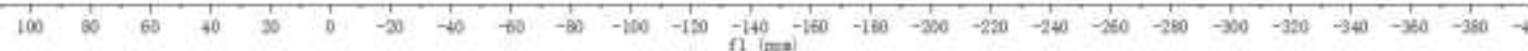




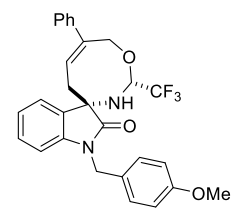

Compound 4na: Yield: $74 \mathrm{mg}$, 75\%; A white solid; Mp: 185-186 ${ }^{\circ} \mathrm{C}$; ${ }^{1} \mathrm{H}$ NMR (400 MHz, Chloroform- $d$ ) $\delta 7.58-7.50(\mathrm{~m}, 2 \mathrm{H}), 7.39(\mathrm{t}, J=8.0 \mathrm{~Hz}, 2 \mathrm{H}), 7.36-7.28(\mathrm{~m}, 1 \mathrm{H}), 7.28-7.11(\mathrm{~m}, 4 \mathrm{H})$, $6.98(\mathrm{t}, J=7.2 \mathrm{~Hz}, 1 \mathrm{H}), 6.88-6.80(\mathrm{~m}, 2 \mathrm{H}), 6.76(\mathrm{~d}, J=8.0 \mathrm{~Hz}, 1 \mathrm{H}), 6.37(\mathrm{t}, J=8.4 \mathrm{~Hz}, 1 \mathrm{H})$, 5.84-5.63 (m, 1H), $4.95(\mathrm{~d}, J=15.2 \mathrm{~Hz}, 1 \mathrm{H}), 4.82(\mathrm{~d}, J=13.2 \mathrm{~Hz}, 1 \mathrm{H}), 4.72(\mathrm{~d}, J=15.2 \mathrm{~Hz}, 1 \mathrm{H})$, $4.66(\mathrm{~d}, J=13.2 \mathrm{~Hz}, 1 \mathrm{H}), 3.76(\mathrm{~s}, 3 \mathrm{H}), 3.06(\mathrm{brs}, 1 \mathrm{H}), 2.61(\mathrm{brs}, 1 \mathrm{H}), 2.48(\mathrm{~d}, J=10.0 \mathrm{~Hz}, 1 \mathrm{H}) ;{ }^{13} \mathrm{C}$ NMR $\left(100 \mathrm{MHz}, \mathrm{CDCl}_{3}\right) \delta 177.0,159.1,141.6,141.2,130.9,129.4,128.6,128.5,127.6,127.4$, 126.4, 125.9, 125.8, $123.0(\mathrm{q}, J=280.0 \mathrm{~Hz}), 122.9,122.4,114.3,114.2,109.7,80.8(\mathrm{q}, J=5.0 \mathrm{~Hz})$, 66.0, 55.2, 42.9, 35.4.; ${ }^{19} \mathrm{~F}$ NMR (376 MHz, Chloroform- $d$ ) $\delta$-80.30; IR (neat): v 3209, 1742, 1496, 1171, 1033, 1064, 834, $715 \mathrm{~cm}^{-1}$; HRMS (ESI) Calcd. for $\mathrm{C}_{28} \mathrm{H}_{26} \mathrm{~F}_{3} \mathrm{~N}_{2} \mathrm{O}_{3}[\mathrm{M}+\mathrm{H}]^{+}:$495.1890, found: 495.1885 .

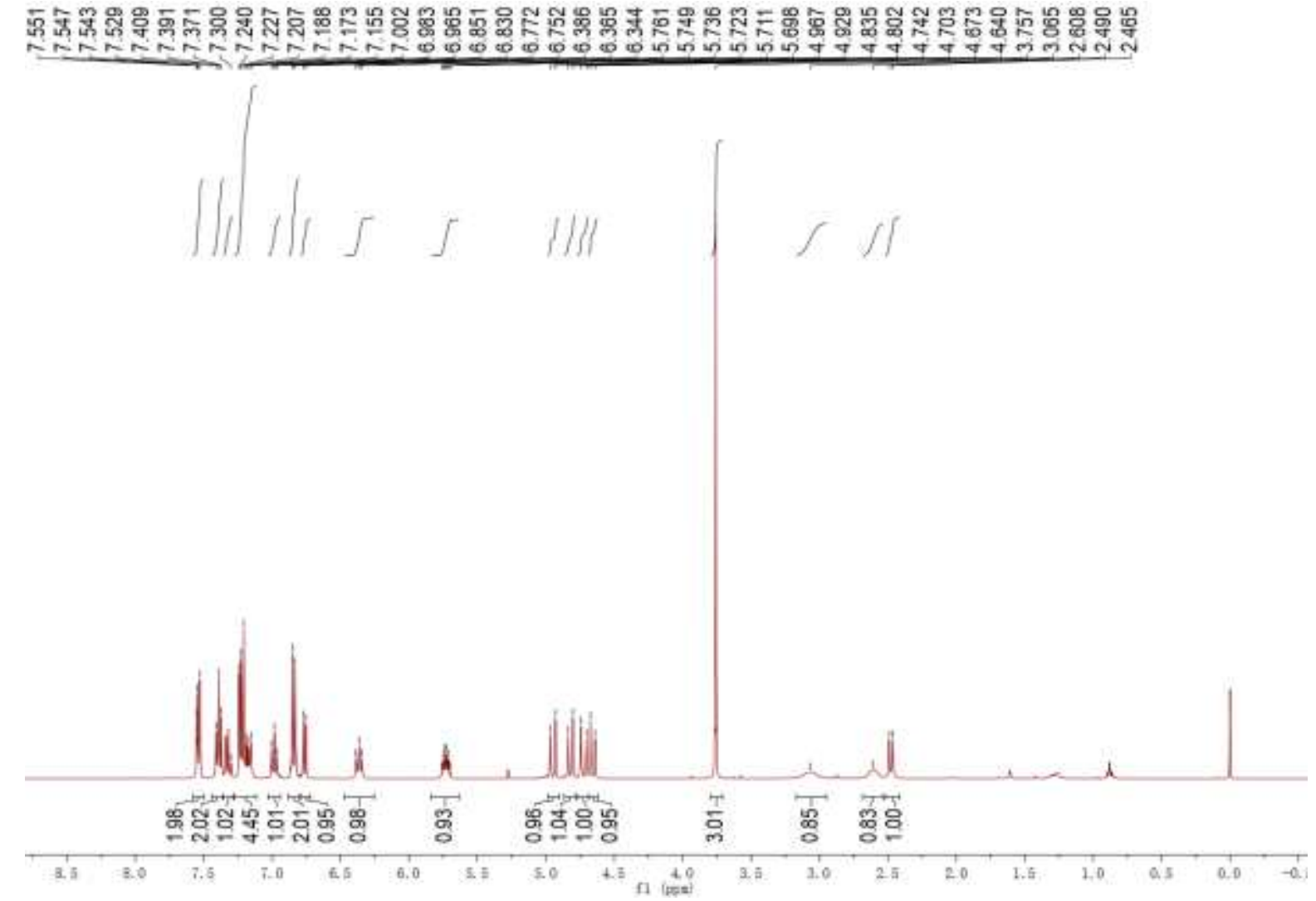




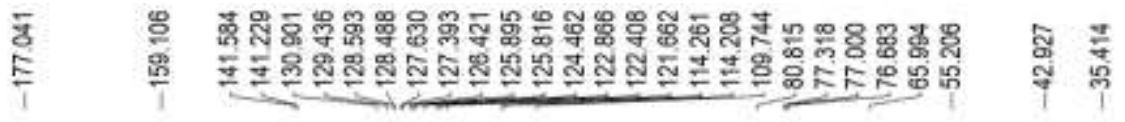
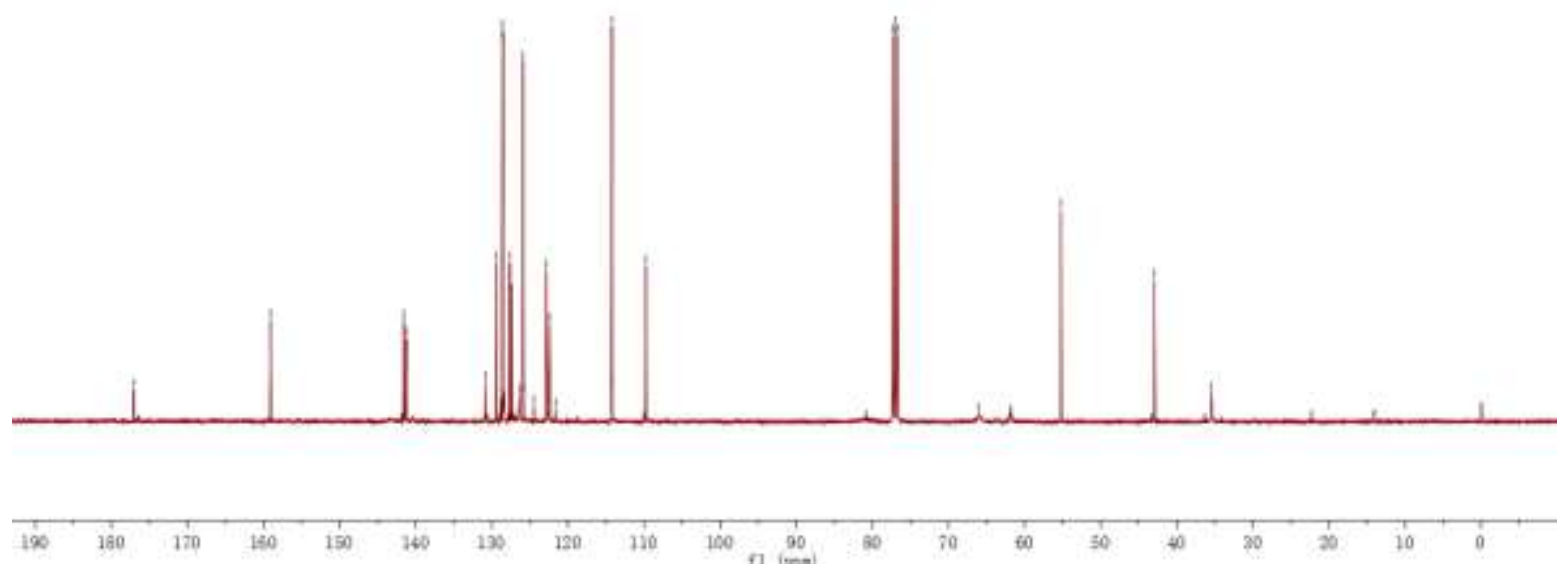

芦

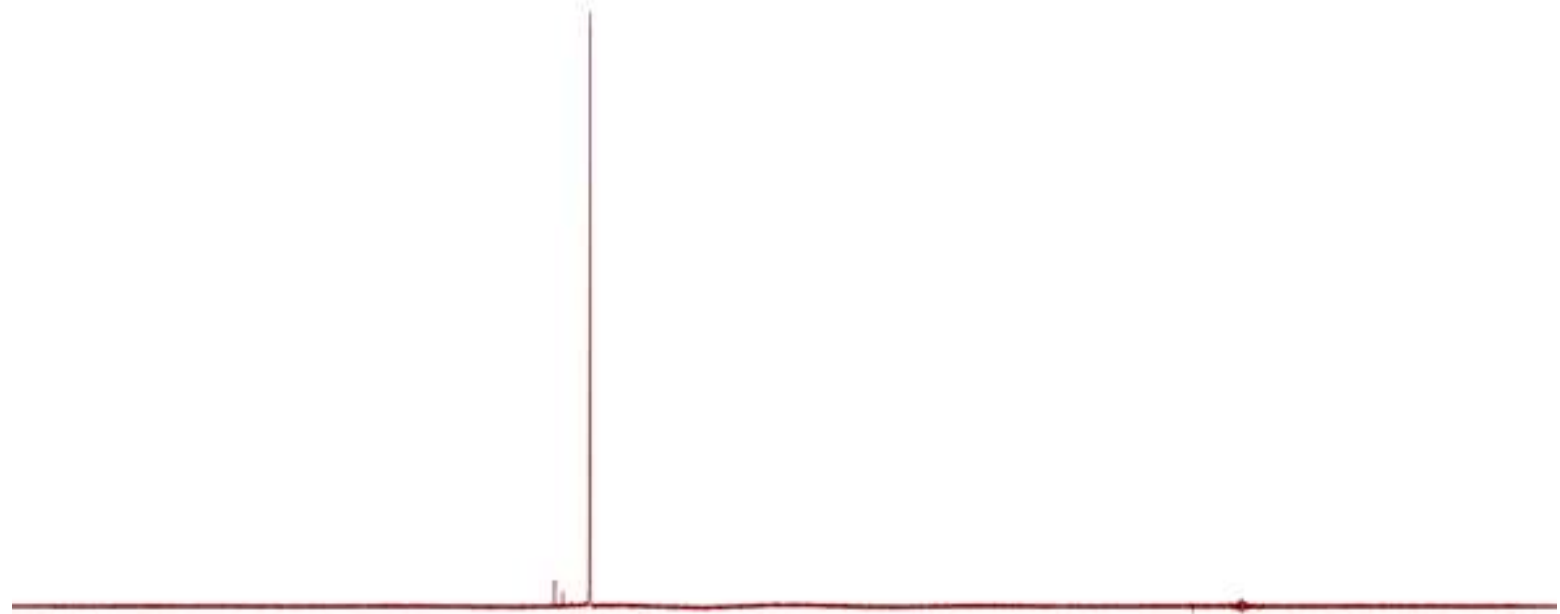

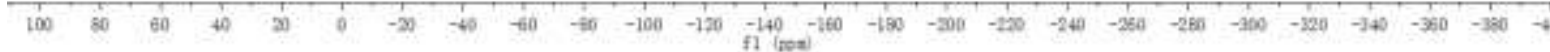


<smiles></smiles>

Compound 4oa: Yield: $55 \mathrm{mg}$, 66\%; A Yellow solid; Mp: 171-173 ${ }^{\circ} \mathrm{C}$; ${ }^{1} \mathrm{H}$ NMR (400 MHz, Chloroform- $d$ ) $\delta$ 7.56-7.49 (m, 2H), 7.43-7.35 (m, 2H), 7.35-7.26 (m, 2H), 7.16 (d, J = 7.2 Hz, 1H), 7.05-6.96 (m, 2H), $6.35(\mathrm{t}, J=8.4 \mathrm{~Hz}, 1 \mathrm{H}), 5.73-5.63(\mathrm{~m}, 1 \mathrm{H}), 4.80(\mathrm{~d}, J=13.2 \mathrm{~Hz}, 1 \mathrm{H}), 4.62$ (d, $J=13.2 \mathrm{~Hz}, 1 \mathrm{H}), 4.56$ (p, $J=7.2 \mathrm{~Hz}, 1 \mathrm{H}), 2.99$ (brs, 1H), 2.55 (brs, 1H), 2.40 (d, $J=10.4 \mathrm{~Hz}$, 1H), $1.49(\mathrm{t}, J=7.2 \mathrm{~Hz}, 6 \mathrm{H}) ;{ }^{13} \mathrm{C} \mathrm{NMR}\left(100 \mathrm{MHz}, \mathrm{CDCl}_{3}\right) \delta 176.7,141.31,141.27,131.3,129.3$, 128.6, 127.6, 126.7, 125.9, 123.0 (q, $J=280.0 \mathrm{~Hz}), 122.6,122.3,110.2,81.0$ (q, $J=5.0 \mathrm{~Hz}), 61.3$, 53.4, 43.9, 35.3, 19.4, 19.3, 19.2; ${ }^{19} \mathrm{~F}$ NMR (376 MHz, Chloroform- $d$ ) $\delta$-80.40; IR (neat): v 3316, 2967, 2068, 1697, 1487, 1196, 759, $750 \mathrm{~cm}^{-1}$; HRMS (ESI) Calcd. for $\mathrm{C}_{23} \mathrm{H}_{24} \mathrm{~F}_{3} \mathrm{~N}_{2} \mathrm{O}_{2}[\mathrm{M}+\mathrm{H}]^{+}$: 417.1784, found: 417.1785 .

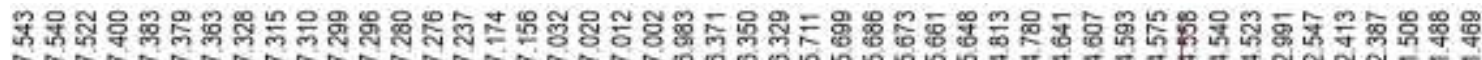

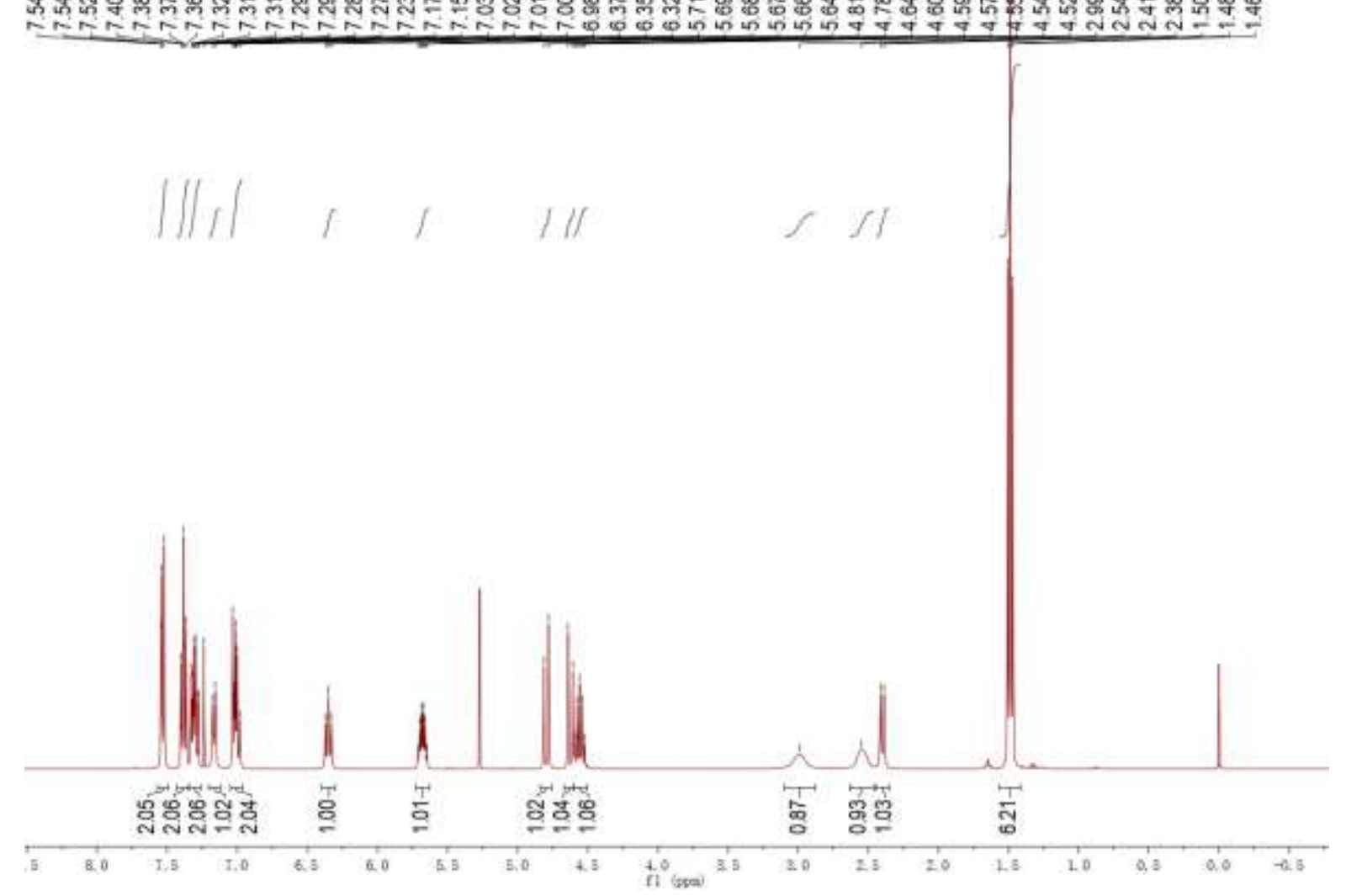




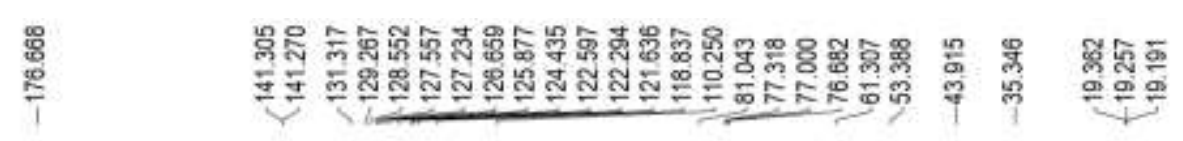
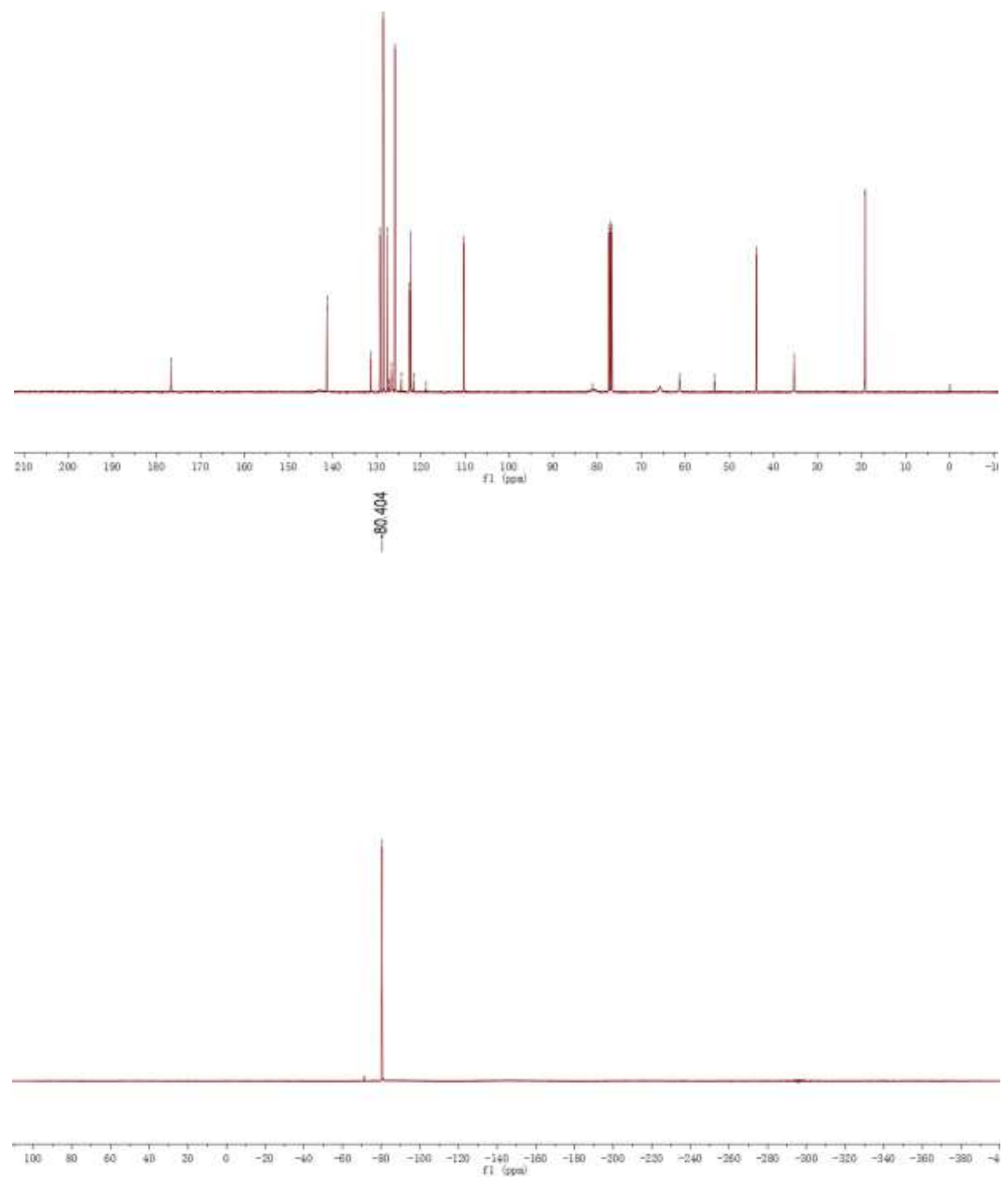

S39 


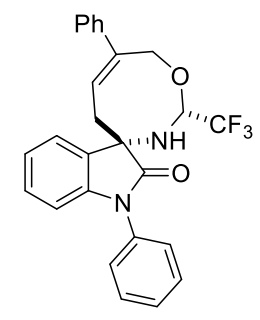

Compound 4pa: Yield: 73mg, 81\%; A White solid; Mp: 164-166 ${ }^{\circ} \mathrm{C}$; ${ }^{1} \mathrm{H}$ NMR (400 MHz, Chloroform- $d$ ) $\delta$ 7.60-7.48 (m, 4H), 7.46-7.35 (m, 4H), 7.34-7.20 (m, 3H), $7.07(\mathrm{t}, J=7.2 \mathrm{~Hz}, 1 \mathrm{H})$, $6.87(\mathrm{~d}, J=7.6 \mathrm{~Hz}, 1 \mathrm{H}), 6.38(\mathrm{t}, J=8.4 \mathrm{~Hz}, 1 \mathrm{H}), 5.76-5.65(\mathrm{~m}, 1 \mathrm{H}), 4.83(\mathrm{~d}, J=13.2 \mathrm{~Hz}, 1 \mathrm{H}), 4.66$ (d, $J=13.2 \mathrm{~Hz}, 1 \mathrm{H}), 3.11$ (brs, $1 \mathrm{H}), 2.73$ (brs, $1 \mathrm{H}), 2.54$ (d, $J=10.4 \mathrm{~Hz}, 1 \mathrm{H}) ;{ }^{13} \mathrm{C} \mathrm{NMR}(100 \mathrm{MHz}$, $\left.\mathrm{CDCl}_{3}\right) \delta 176.3,142.5,141.2,133.8,130.6,129.6,129.5,128.6,128.2,127.6,126.4,125.9,123.4$, $123.1(\mathrm{q}, J=280.1 \mathrm{~Hz}), 122.7,110.0,80.5(\mathrm{q}, J=5.0 \mathrm{~Hz}), 66.2,61.8,35.5 ;{ }^{19} \mathrm{~F} \mathrm{NMR}(376 \mathrm{MHz}$, Chloroform- $d$ ) $\delta$-80.21; IR (neat): v 3382, 3060, 1720, 1612, 1498, 1150, 748, $698 \mathrm{~cm}^{-1}$; HRMS (ESI) Calcd. for $\mathrm{C}_{26} \mathrm{H}_{21} \mathrm{~F}_{3} \mathrm{~N}_{2} \mathrm{O}_{2}[\mathrm{M}+\mathrm{H}]^{+}:$: 451.1628, found: 451.1639.

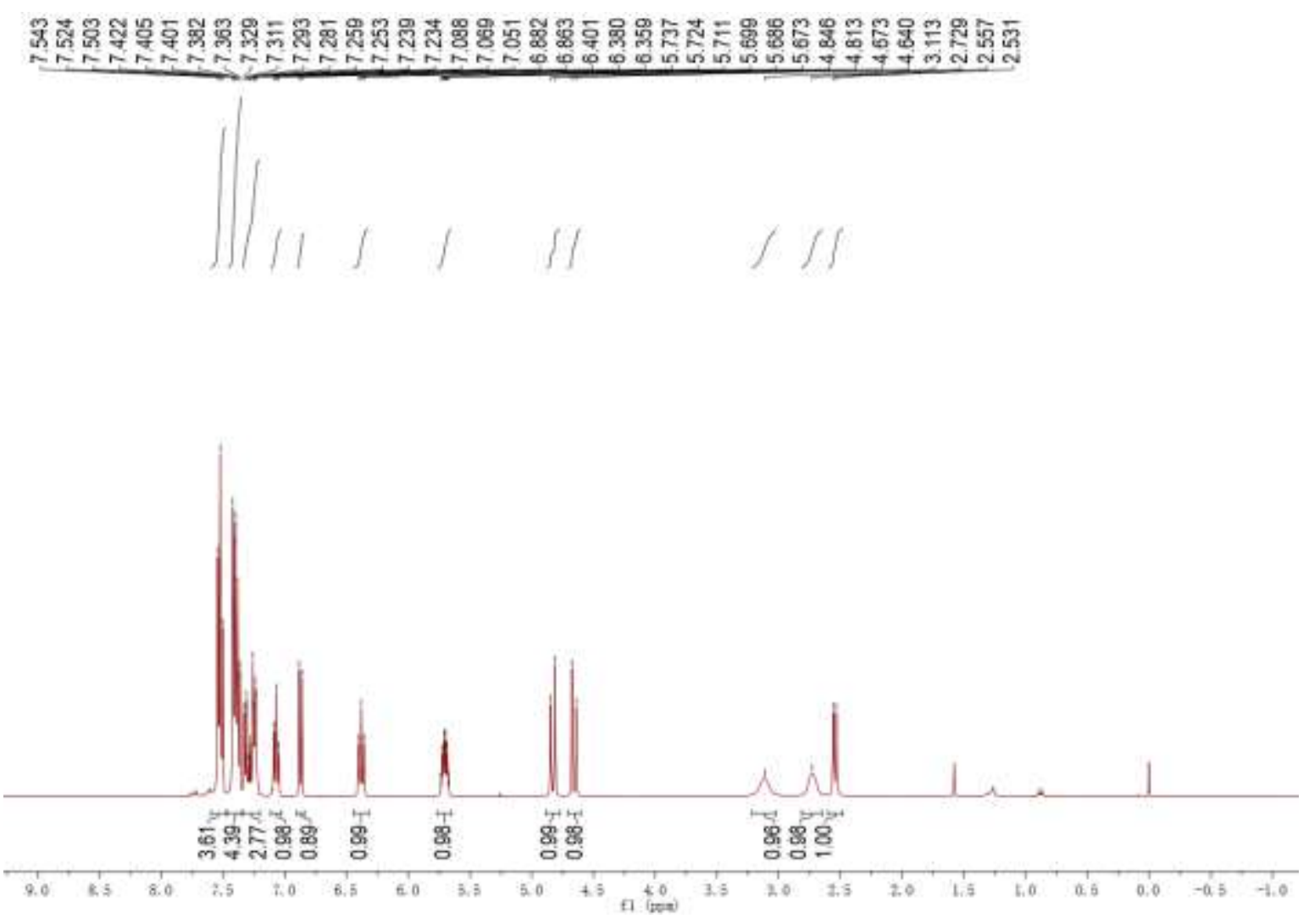



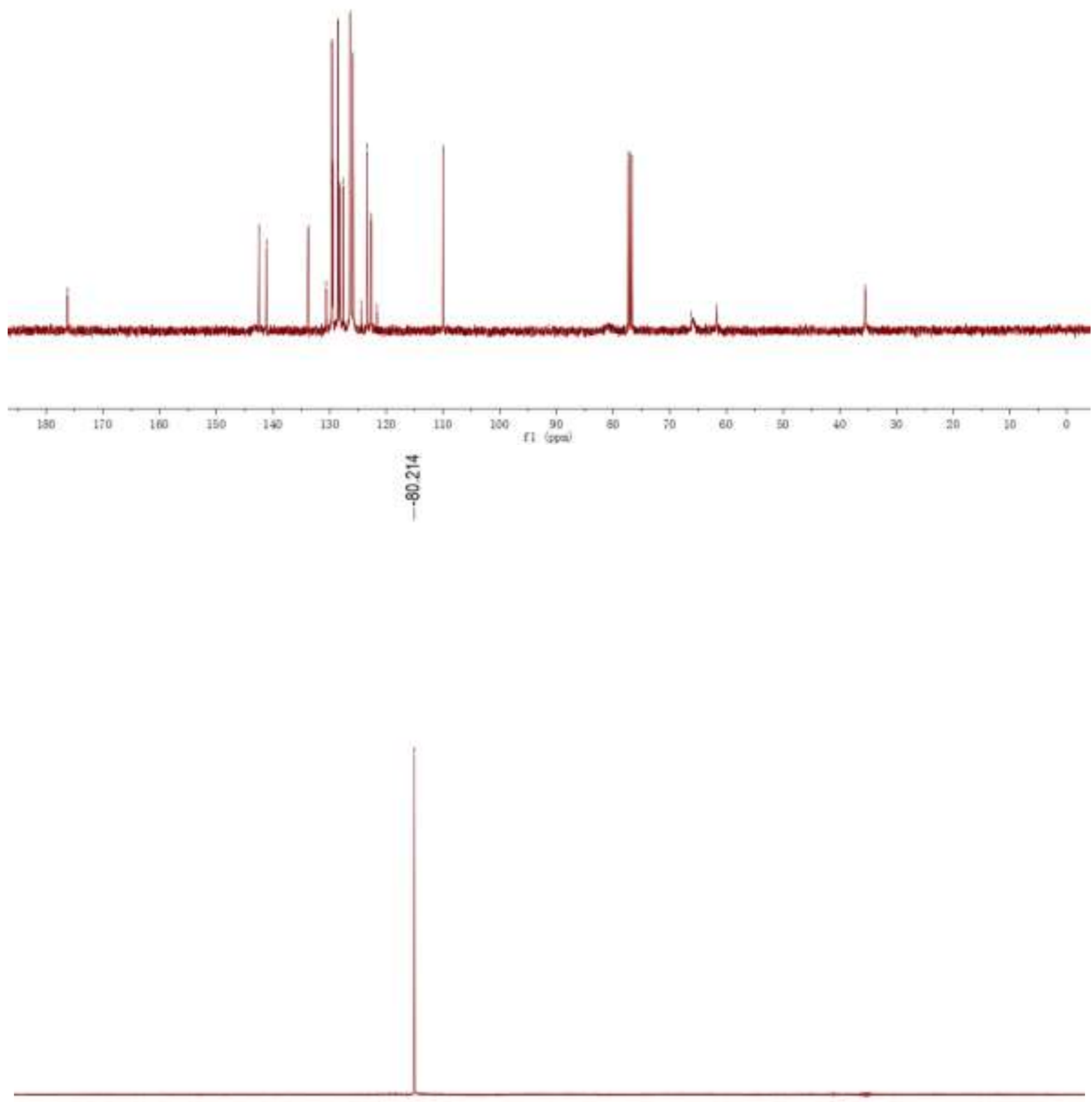

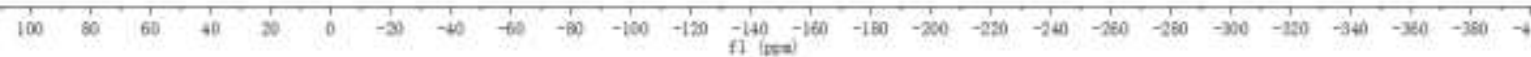




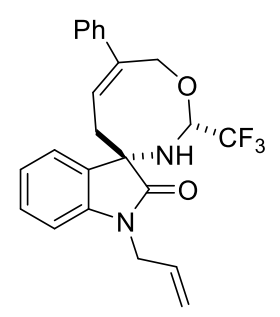

Compound 4qa: Yield: $73 \mathrm{mg}$, 85\%; A white foam solid; Mp: 166-167 ${ }^{\circ} \mathrm{C} ;{ }^{1} \mathrm{H}$ NMR $(400 \mathrm{MHz}$, Chloroform- $d$ ) $\delta 7.65-7.50(\mathrm{~m}, 2 \mathrm{H}), 7.49-7.14(\mathrm{~m}, 5 \mathrm{H}), 7.05$ (t, $J=7.6 \mathrm{~Hz}, 1 \mathrm{H}), 6.88(\mathrm{~d}, J=8.0 \mathrm{~Hz}$, $1 \mathrm{H}), 6.37(\mathrm{t}, J=8.4 \mathrm{~Hz}, 1 \mathrm{H}), 5.96-5.78(\mathrm{~m}, 1 \mathrm{H}), 5.77-5.61(\mathrm{~m}, 1 \mathrm{H}), 5.36-5.12(\mathrm{~m}, 2 \mathrm{H}), 4.83(\mathrm{~d}, J$ $=13.2 \mathrm{~Hz}, 1 \mathrm{H}), 4.67(\mathrm{~d}, J=13.2 \mathrm{~Hz}, 1 \mathrm{H}), 4.47-4.25(\mathrm{~m}, 2 \mathrm{H}), 3.07$ (brs, $1 \mathrm{H}), 2.61(\mathrm{brs}, 1 \mathrm{H}), 2.50(\mathrm{~d}$, $J=10.0 \mathrm{~Hz}, 1 \mathrm{H}) ;{ }^{13} \mathrm{C} \mathrm{NMR}\left(100 \mathrm{MHz}, \mathrm{CDCl}_{3}\right) \delta 176.7,141.7,141.2,130.9,130.8,129.4,128.6$, 127.6, 126.3, 125.9, 123.0 (q, $J=280.4 \mathrm{~Hz}), 122.8,122.4,117.5,109.6,80.8(\mathrm{q}, J=6.0 \mathrm{~Hz}), 66.0$, 61.7, 42.0, 35.4; ${ }^{19} \mathrm{~F}$ NMR (376 MHz, Chloroform- $d$ ) $\delta$-80.41; IR (neat): v 3421, 1705, 1611, 1132, 1038, $758 \mathrm{~cm}^{-1}$; HRMS (ESI) Calcd. for $\mathrm{C}_{23} \mathrm{H}_{22} \mathrm{~F}_{3} \mathrm{~N}_{2} \mathrm{O}_{2}[\mathrm{M}+\mathrm{H}]^{+}: 415.1628$, found: 415.1633 .

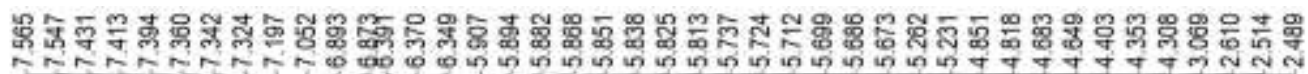

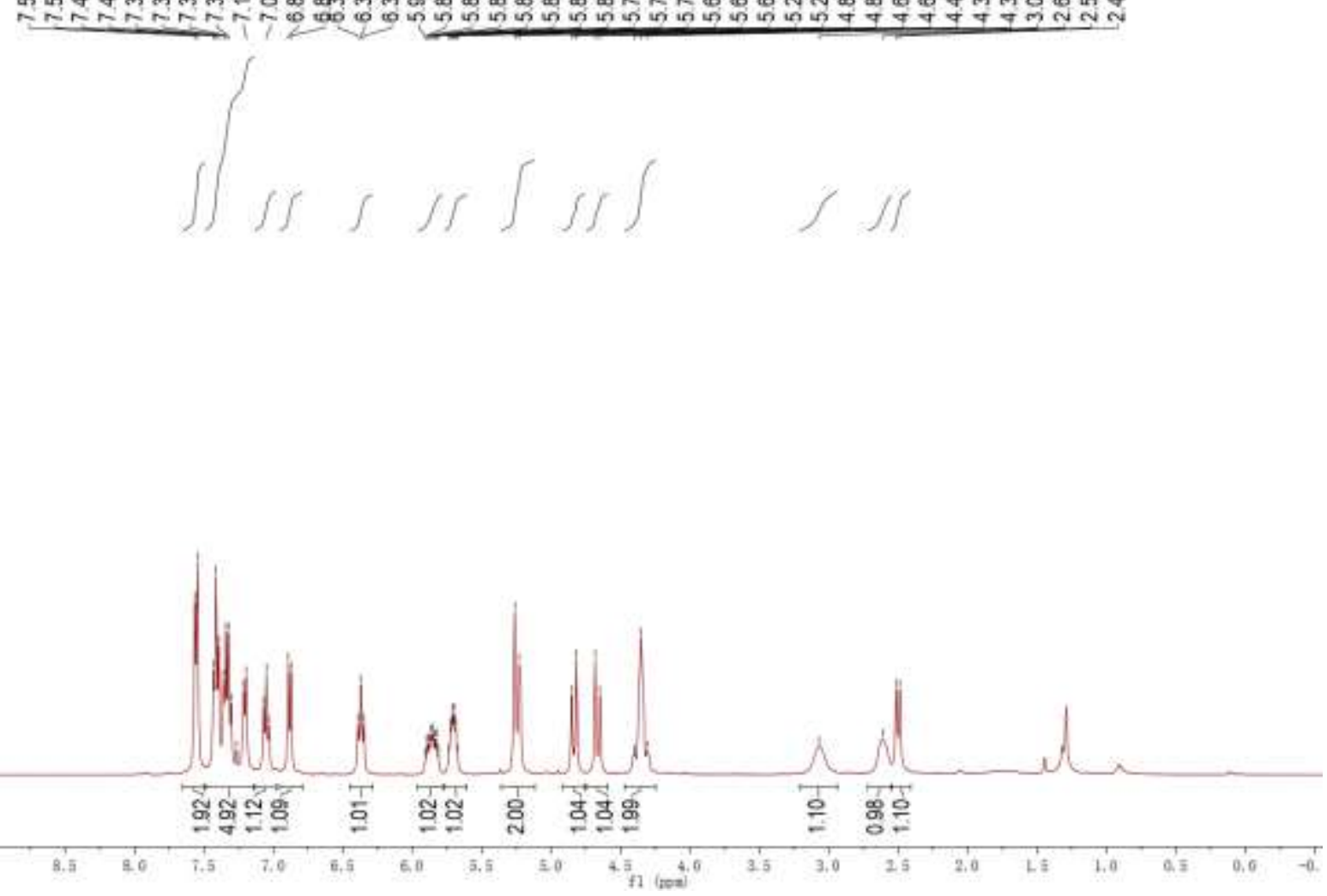



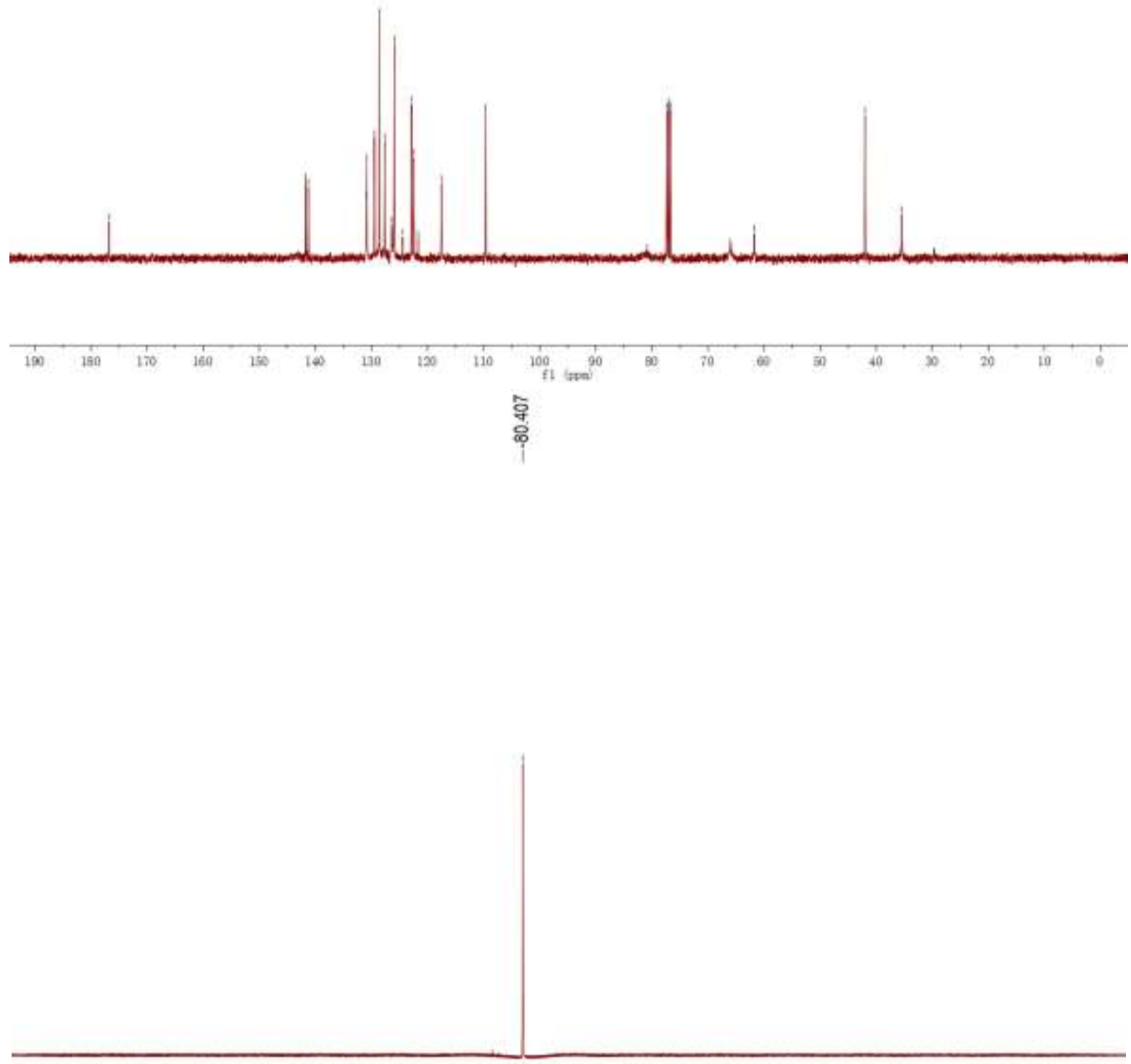

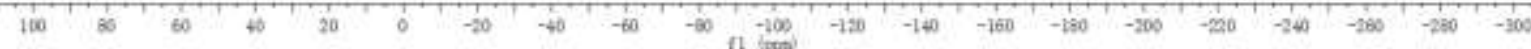


<smiles>O=C1Nc2ccccc2[C@@]12C/C=C(\c1ccccc1)COC(C(F)(F)F)N2</smiles>

Compound 4ra: Yield: $20 \mathrm{mg}, 27 \%$; A yellow foam solid; ${ }^{1} \mathrm{H}$ NMR (400 MHz, Chloroform- $d$ ) $\delta$ $8.46(\mathrm{~s}, 1 \mathrm{H}), 7.64-7.47(\mathrm{~m}, 2 \mathrm{H}), 7.46-7.22(\mathrm{~m}, 4 \mathrm{H}), 7.21-7.10(\mathrm{~m}, 1 \mathrm{H}), 7.10-6.97(\mathrm{~m}, 1 \mathrm{H})$, 6.97-6.83 (m, 1H), 6.43-6.24 (m, 1H), $5.66(\mathrm{~s}, 1 \mathrm{H}), 4.82$ (d, J=13.2 Hz, 1H), 4.64 (d, J = 13.2 Hz, 1H), $3.02(\mathrm{~s}, 1 \mathrm{H}), 2.63(\mathrm{~s}, 1 \mathrm{H}), 2.50(\mathrm{~d}, J=10.8 \mathrm{~Hz}, 1 \mathrm{H}) ;{ }^{13} \mathrm{C} \mathrm{NMR}\left(100 \mathrm{MHz}, \mathrm{CDCl}_{3}\right) \delta 179.7$, $141.2,139.7,131.3,129.6,128.6,127.7,126.3,125.9,123.1$ (q, $J=280.0 \mathrm{~Hz}) 122.9,122.7,110.6$, 81.2(q, $J=6.0 \mathrm{~Hz}), 66.0,62.1,35.5 ;{ }^{19} \mathrm{~F}$ NMR (376 MHz, Chloroform- $d$ ) $\delta$-80.29; IR (neat): $v$ 3348, 1709, 1492, 1144, 1059, 1001, 975, $702 \mathrm{~cm}^{-1}$; HRMS (ESI) Calcd. for $\mathrm{C}_{20} \mathrm{H}_{18} \mathrm{~F}_{3} \mathrm{~N}_{2} \mathrm{O}_{2}[\mathrm{M}+\mathrm{H}]^{+}$: 375.1315, found: 315.1319 .
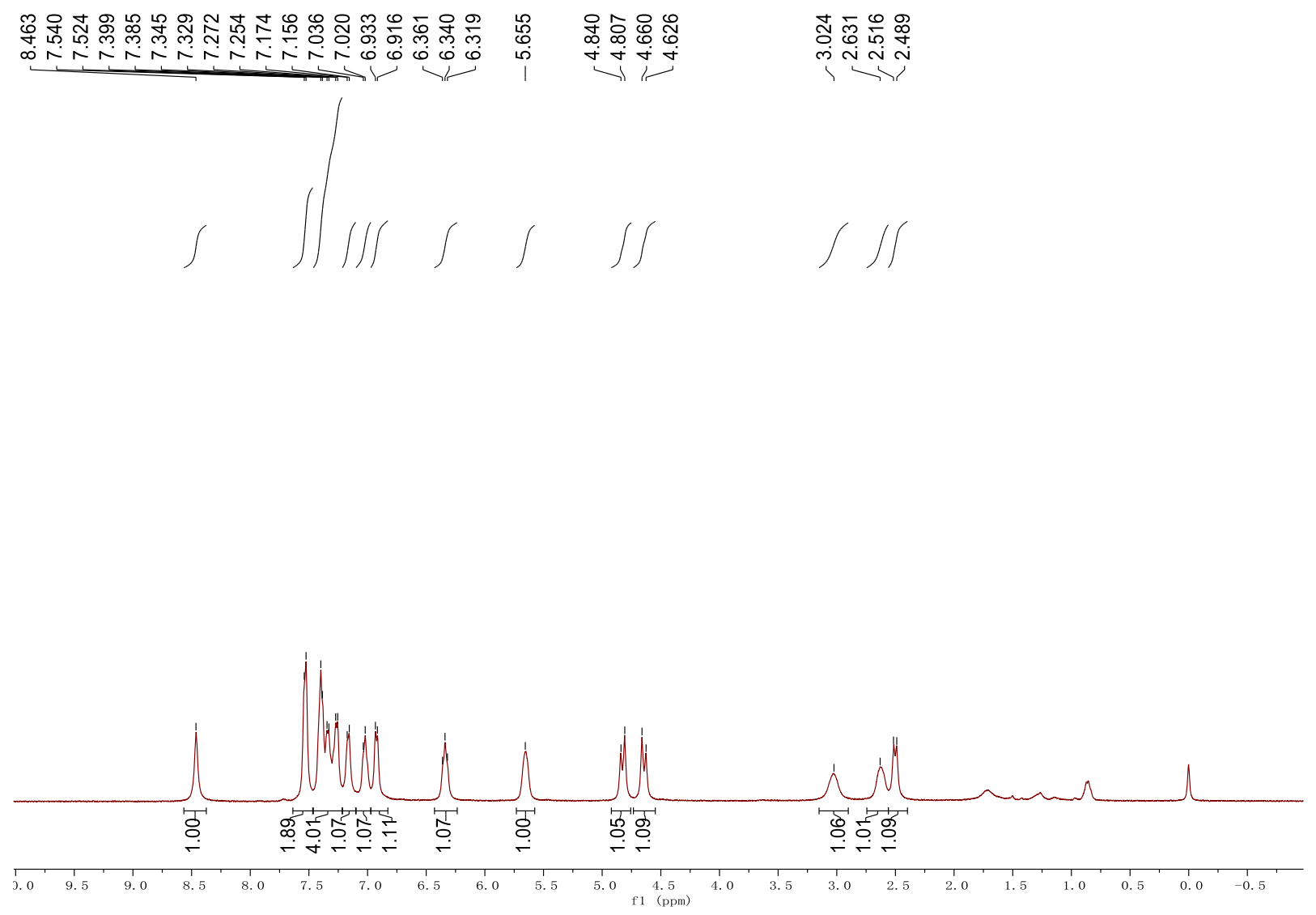

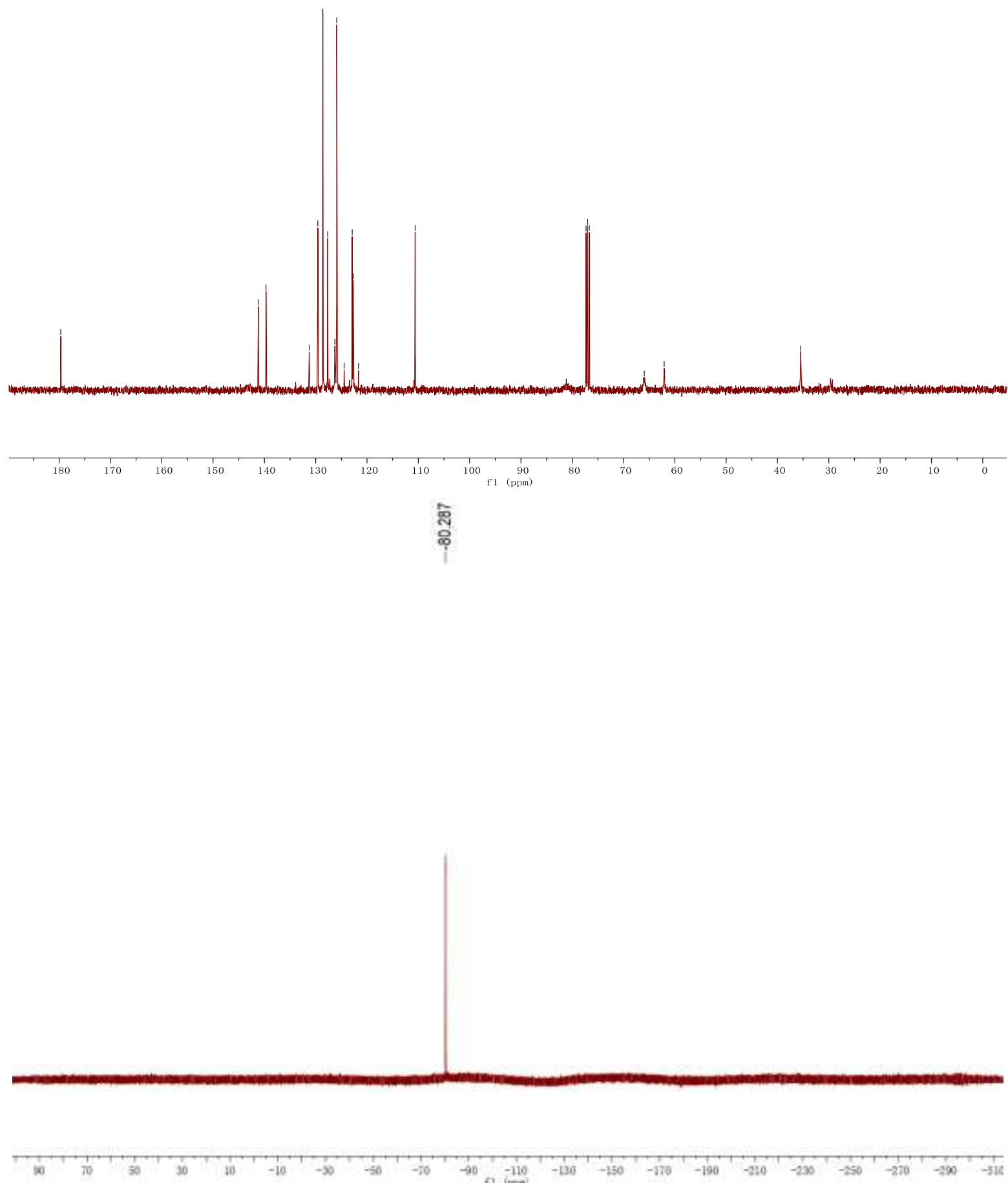


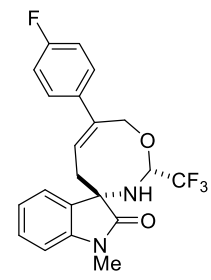

Compound 4ab: Yield: $61 \mathrm{mg}$, 75\%; A White foam solide; Mp: 165-166 ${ }^{\circ} \mathrm{C}$; ${ }^{1} \mathrm{H}$ NMR (400 MHz, Chloroform- $d$ ) $\delta 7.58-7.41(\mathrm{~m}, 2 \mathrm{H}), 7.35(\mathrm{t}, J=7.6 \mathrm{~Hz}, 1 \mathrm{H}), 7.17(\mathrm{~d}, J=7.2 \mathrm{~Hz}, 1 \mathrm{H}), 7.12-6.96(\mathrm{~m}$, $3 \mathrm{H}), 6.88(\mathrm{~d}, J=8.0 \mathrm{~Hz}, 1 \mathrm{H}), 6.27(\mathrm{t}, J=8.4 \mathrm{~Hz}, 1 \mathrm{H}), 5.74-5.56(\mathrm{~m}, 1 \mathrm{H}), 4.77(\mathrm{~d}, J=13.2 \mathrm{~Hz}, 1 \mathrm{H})$, $4.61(\mathrm{~d}, J=13.2 \mathrm{~Hz}, 1 \mathrm{H}), 3.21$ (s, 3H), 2.97 (brs, 1H), 2.57 (brs, $1 \mathrm{H}), 2.47$ (d, $J=10.0 \mathrm{~Hz}, 1 \mathrm{H}) ;{ }^{13} \mathrm{C}$ NMR $\left(100 \mathrm{MHz}, \mathrm{CDCl}_{3}\right) \delta 176.9,162.4(\mathrm{~d}, J=245.3 \mathrm{~Hz}), 142.5,137.3(\mathrm{~d}, J=3.2 \mathrm{~Hz}), 130.8$, 129.6, $127.6(\mathrm{~d}, J=8.0 \mathrm{~Hz}), 126.4,123.0(\mathrm{q}, J=280.2 \mathrm{~Hz}), 122.9,122.3,115.4(\mathrm{~d}, J=21.3 \mathrm{~Hz})$, 108.8, 80.8 (q, $J=5.0 \mathrm{~Hz}), 65.8,61.6,35.6,26.1 ;{ }^{19} \mathrm{~F}$ NMR (376 MHz, Chloroform- $d$ ) $\delta-80.28$, -114.70; IR (neat): v 3327, 2903, 1803, 1692, 1508, 1174, 1057, 832, $746 \mathrm{~cm}^{-1}$; HRMS (ESI) Calcd. for $\mathrm{C}_{21} \mathrm{H}_{19} \mathrm{~F}_{4} \mathrm{~N}_{2} \mathrm{O}_{2}[\mathrm{M}+\mathrm{H}]^{+}:$407.1377, found: 407.1378.

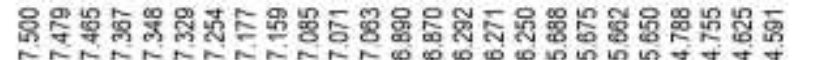
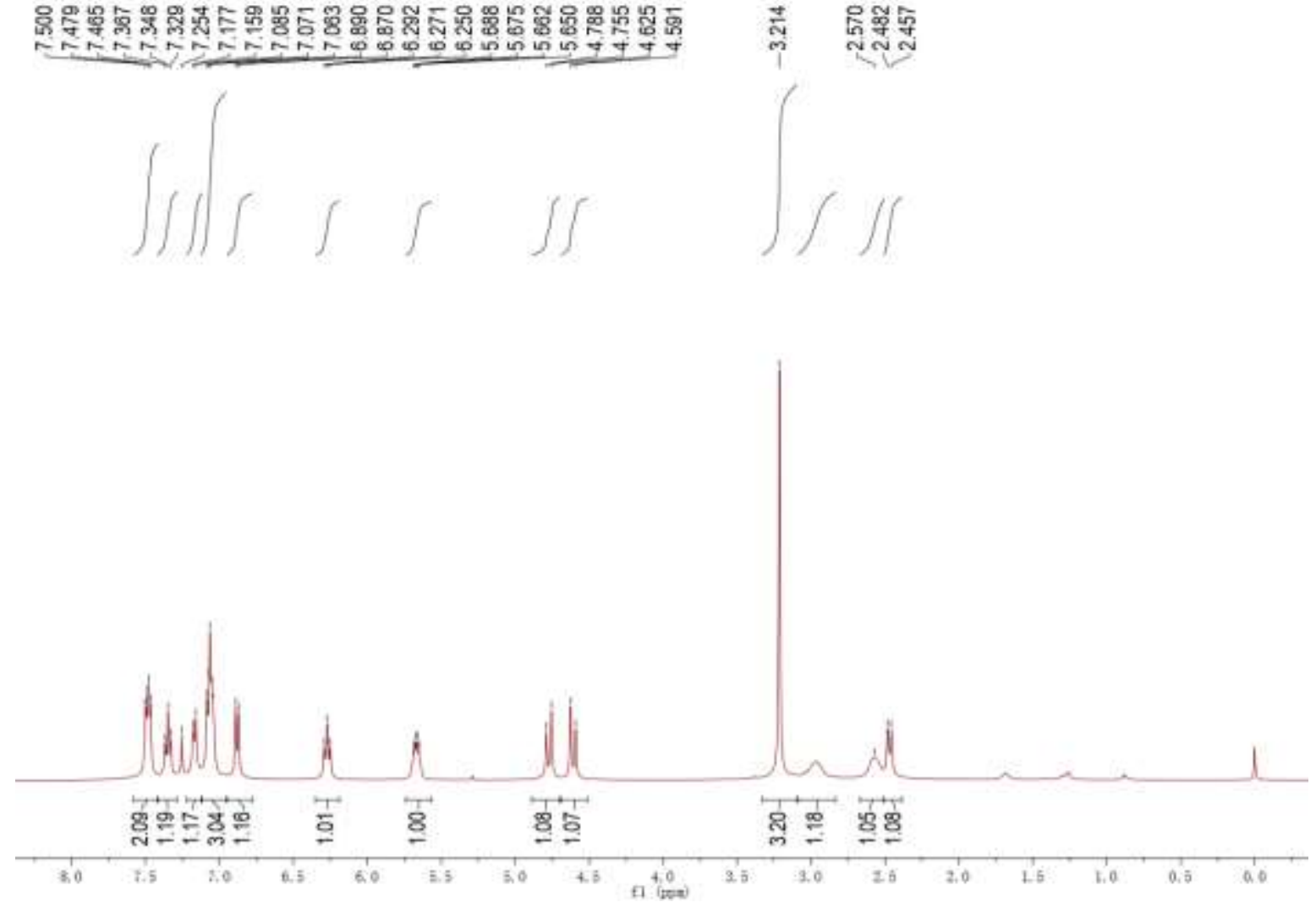


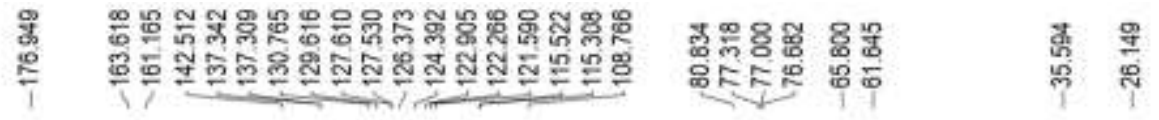
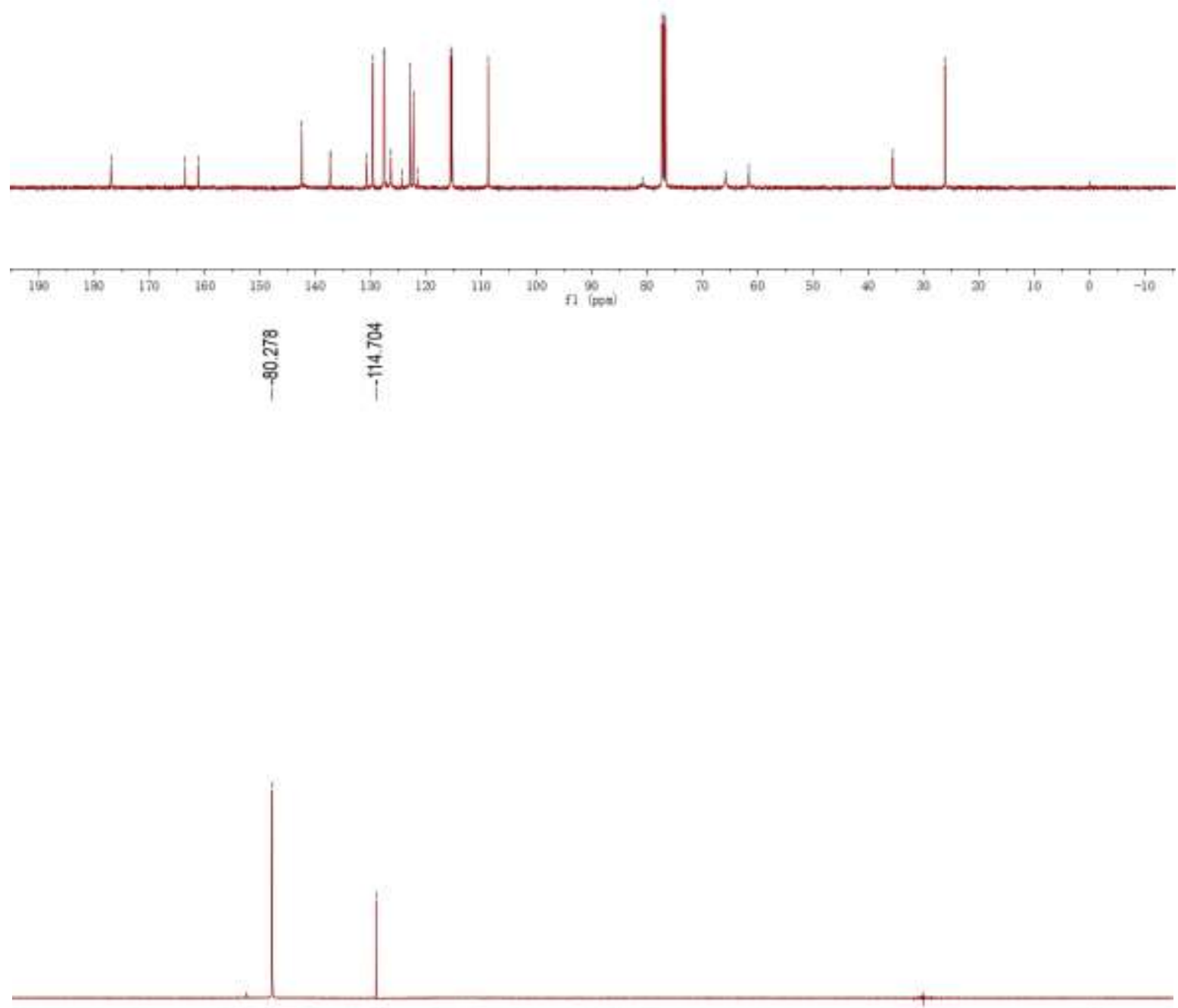

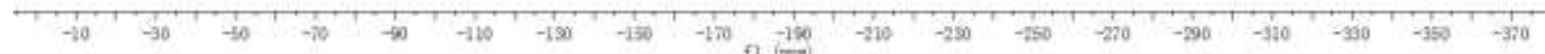




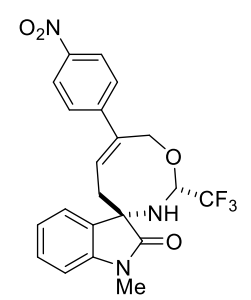

Compound 4ac: Yield: $50 \mathrm{mg}$, 58\%; A Yellow solide; Mp: 205-207 ${ }^{\circ} \mathrm{C}$; ${ }^{1} \mathrm{H}$ NMR (400 MHz, Acetone- $\left.d_{6}\right) \delta 8.28(\mathrm{~d}, J=8.8 \mathrm{~Hz}, 2 \mathrm{H}), 7.84(\mathrm{~d}, J=8.8 \mathrm{~Hz}, 2 \mathrm{H}), 7.52(\mathrm{~d}, J=7.6 \mathrm{~Hz}, 1 \mathrm{H}), 7.37(\mathrm{t}, J$ $=7.2 \mathrm{~Hz}, 1 \mathrm{H}), 7.18-6.94(\mathrm{~m}, 2 \mathrm{H}), 6.70(\mathrm{t}, J=8.4 \mathrm{~Hz}, 1 \mathrm{H}), 5.78-5.70(\mathrm{~m}, 1 \mathrm{H}), 4.94(\mathrm{~d}, J=13.2 \mathrm{~Hz}$, 1H), 4.74 (d, $J=13.2 \mathrm{~Hz}, 1 \mathrm{H}), 3.63$ (d, $J=10.2 \mathrm{~Hz}, 1 \mathrm{H}), 3.20$ (s, 3H), 2.86 (brs, 2H); ${ }^{13} \mathrm{C}$ NMR $(100 \mathrm{MHz}$, Acetone $) \delta 177.1,148.8,147.9,143.4,133.0,131.9,130.1,127.7,124.5,124.4(\mathrm{q}, J=$ $279.3 \mathrm{~Hz}), 123.9,123.3,109.4,80.0$ (q, $J=5.0 \mathrm{~Hz}), 79.1,64.0,62.4,36.9,26.2 ;{ }^{19} \mathrm{~F}$ NMR $(376$ MHz, Deuterium Oxide) $\delta$-77.82; IR (neat): v 3474, 3400, 1699, 1610, 1343, 1187, 1131, 854, 686 $\mathrm{cm}^{-1}$; HRMS (ESI) Calcd. for $\mathrm{C}_{21} \mathrm{H}_{19} \mathrm{~F}_{3} \mathrm{~N}_{3} \mathrm{O}_{4}[\mathrm{M}+\mathrm{H}]^{+}:$434.1322, found: 434.1315.
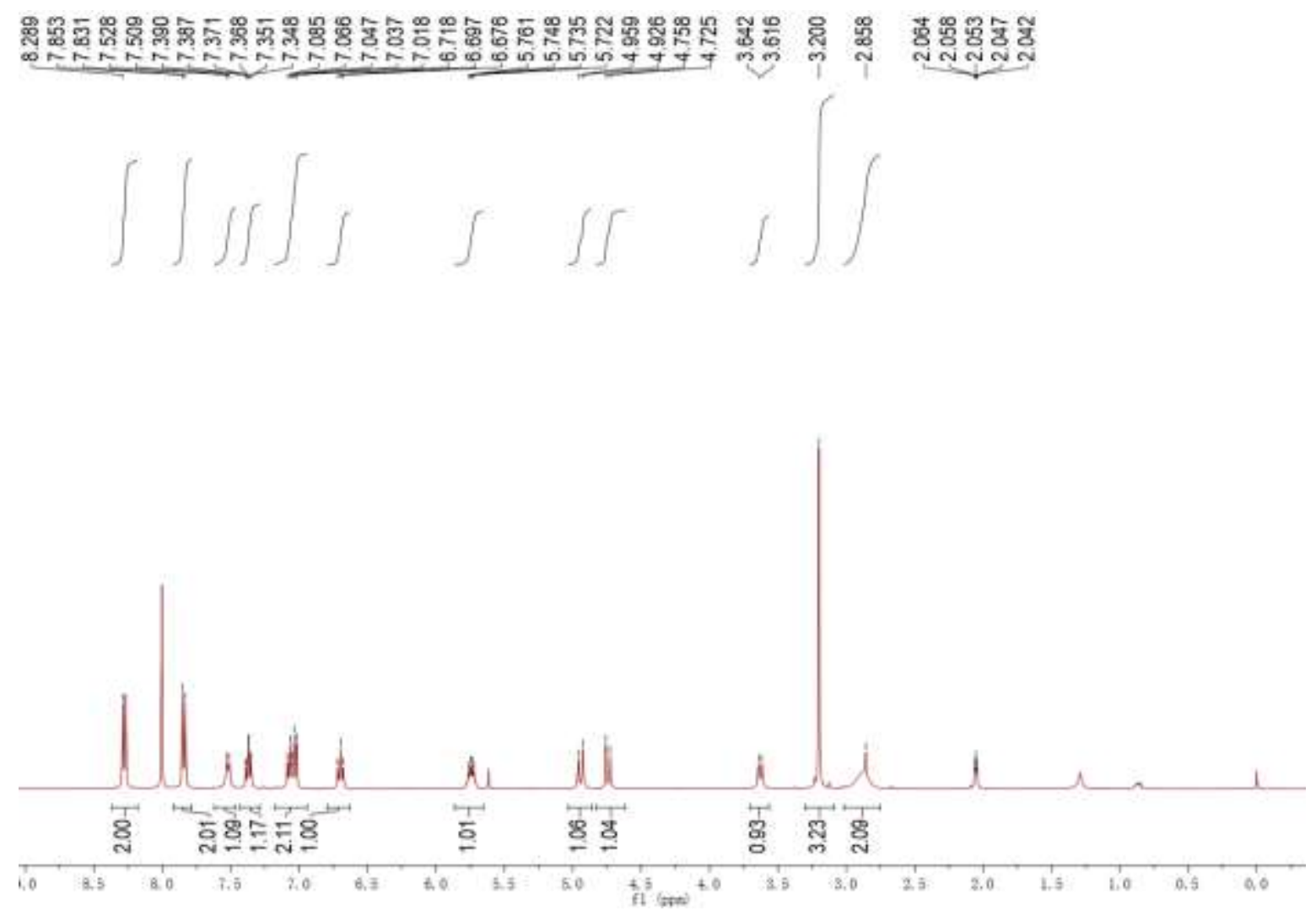

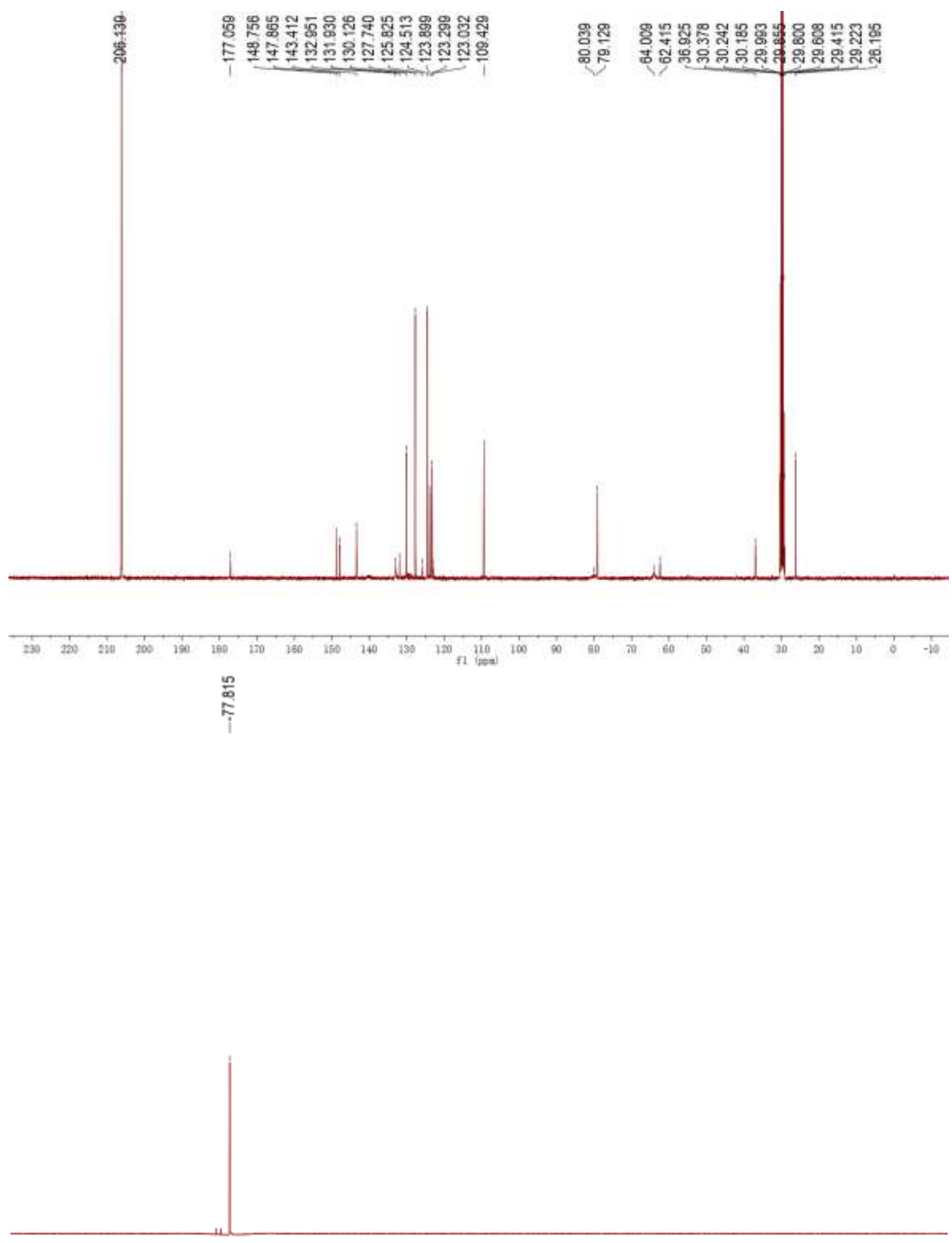

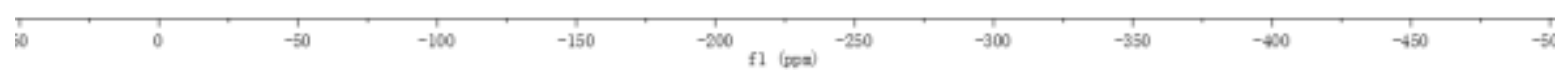




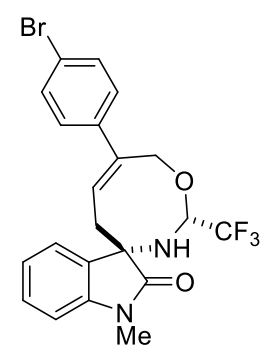

Compound 4ad: Yield: $74 \mathrm{mg}, 79 \%$; A White solid; Mp: 190-192 ${ }^{\circ} \mathrm{C}$; ${ }^{1} \mathrm{H}$ NMR (400 MHz, Chloroform- $d$ ) $\delta 7.50(\mathrm{~d}, J=8.4 \mathrm{~Hz}, 2 \mathrm{H}), 7.45-7.30(\mathrm{~m}, 3 \mathrm{H}), 7.20-7.10(\mathrm{~m}, 1 \mathrm{H}), 7.05(\mathrm{t}, J=7.6 \mathrm{~Hz}$, $1 \mathrm{H}), 6.88(\mathrm{~d}, J=8.0 \mathrm{~Hz}, 1 \mathrm{H}), 6.32(\mathrm{t}, J=8.4 \mathrm{~Hz}, 1 \mathrm{H}), 5.71-5.59(\mathrm{~m}, 1 \mathrm{H}), 4.76(\mathrm{~d}, J=13.2 \mathrm{~Hz}, 1 \mathrm{H})$, $4.60(\mathrm{~d}, J=13.2 \mathrm{~Hz}, 1 \mathrm{H}), 3.22(\mathrm{~s}, 3 \mathrm{H}), 2.97$ (brs, $1 \mathrm{H}), 2.57$ (brs, $1 \mathrm{H}), 2.45(\mathrm{~d}, J=10.0 \mathrm{~Hz}, 1 \mathrm{H}) ;{ }^{13} \mathrm{C}$ $\operatorname{NMR}\left(100 \mathrm{MHz}, \mathrm{CDCl}_{3}\right) \delta 176.9,142.5,140.1,131.7,130.7,129.7,127.5,127.2,123.0(\mathrm{q}, J=$ $279.7 \mathrm{~Hz}), 122.9,122.3,121.7,108.8,80.6(\mathrm{q}, J=7.0 \mathrm{~Hz}), 65.6,61.6,35.6,26.2 ;{ }^{19} \mathrm{~F}$ NMR $(376$ MHz, Chloroform- $d$ ) $\delta$-80.23; IR (neat): $v$ 3322, 2976, 2872, 1704, 1492, 1175, 1134, 817, 739 $\mathrm{cm}^{-1}$; HRMS (ESI) Calcd. for $\mathrm{C}_{21} \mathrm{H}_{19} \mathrm{BrF}_{3} \mathrm{~N}_{2} \mathrm{O}_{2}[\mathrm{M}+\mathrm{H}]^{+}$: 467.0577, found: 467.0579.

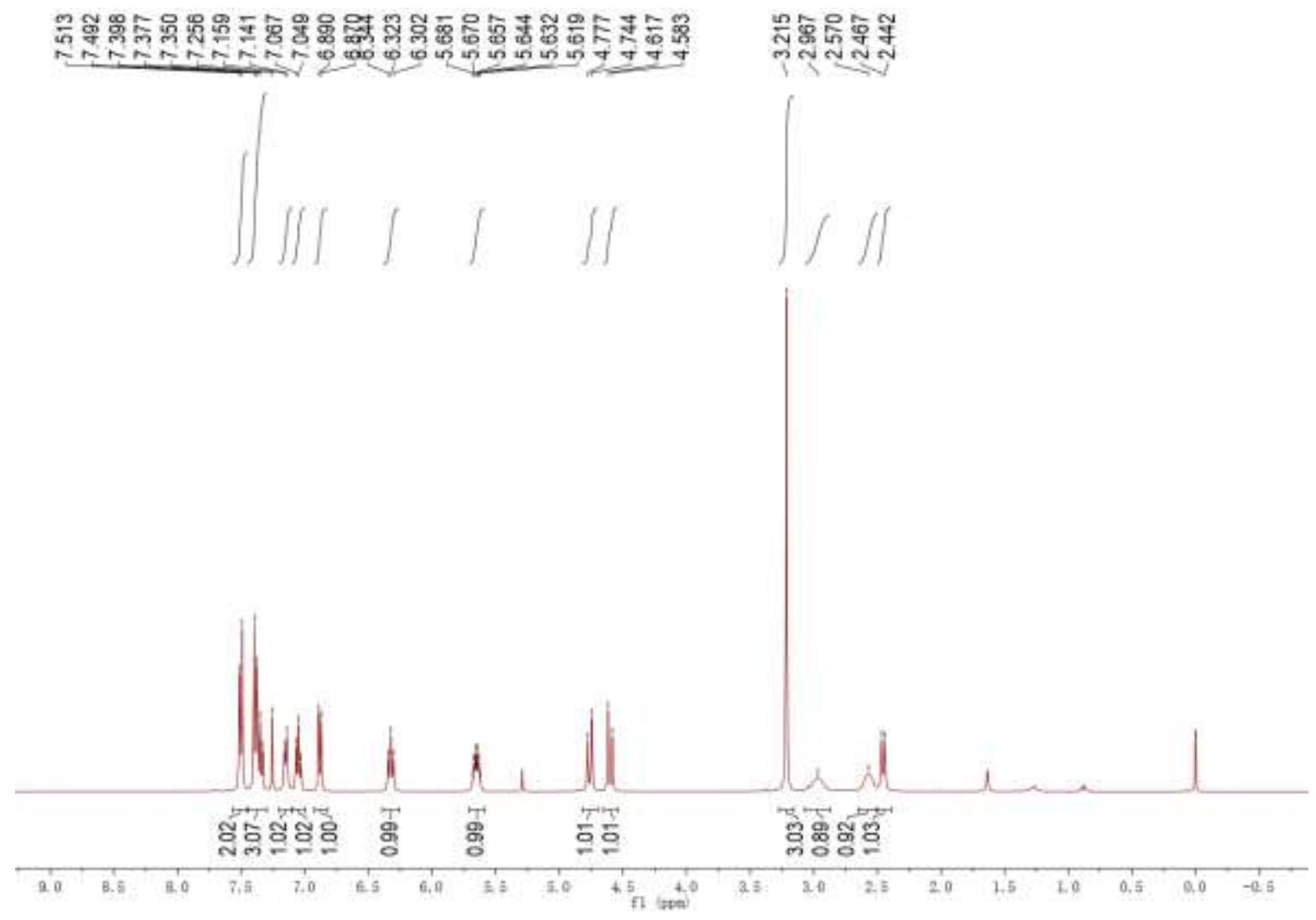




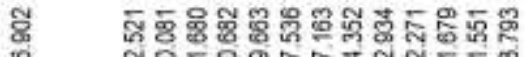

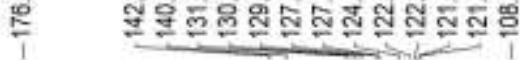

웅

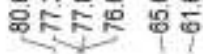

总 爱
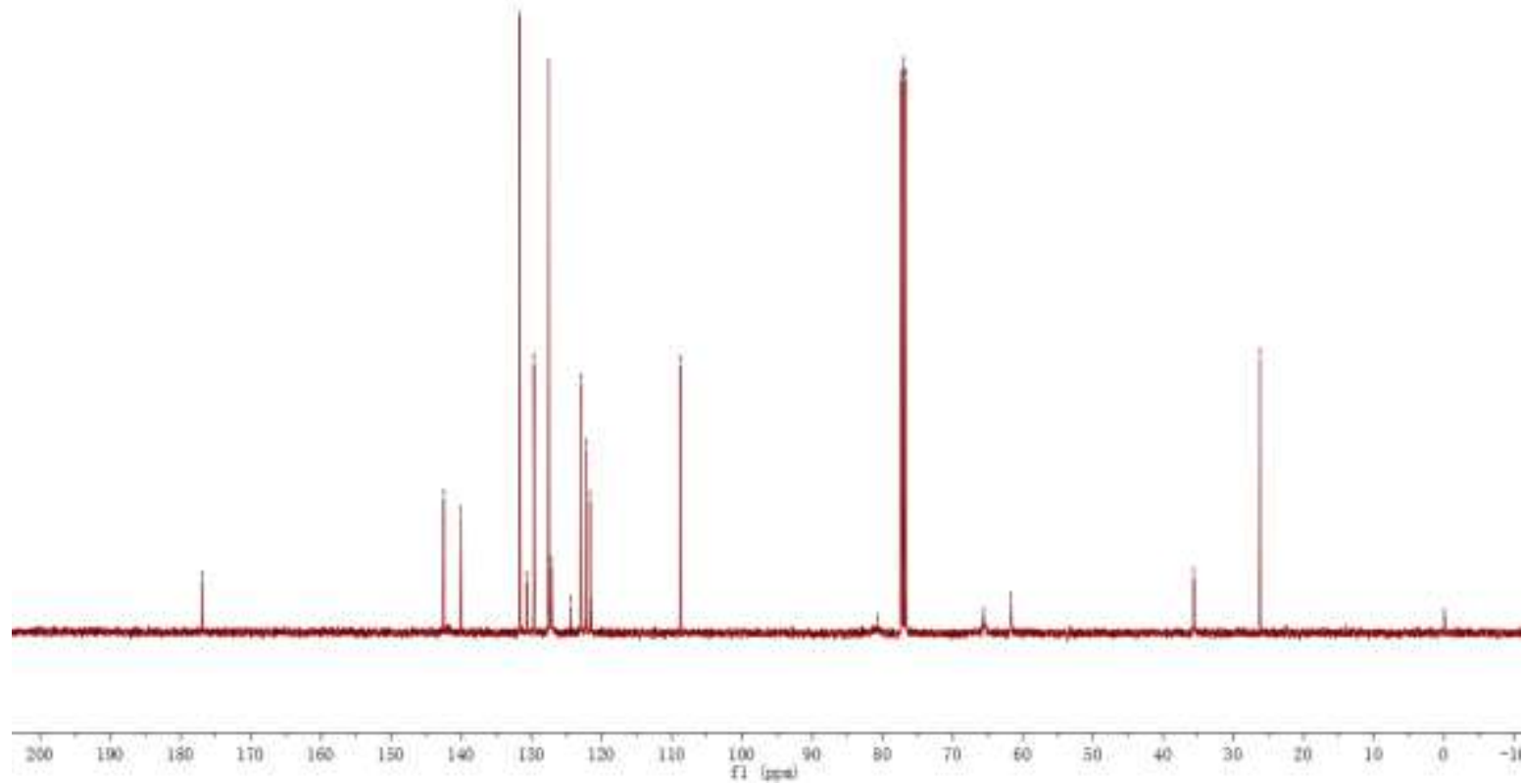

בัּ

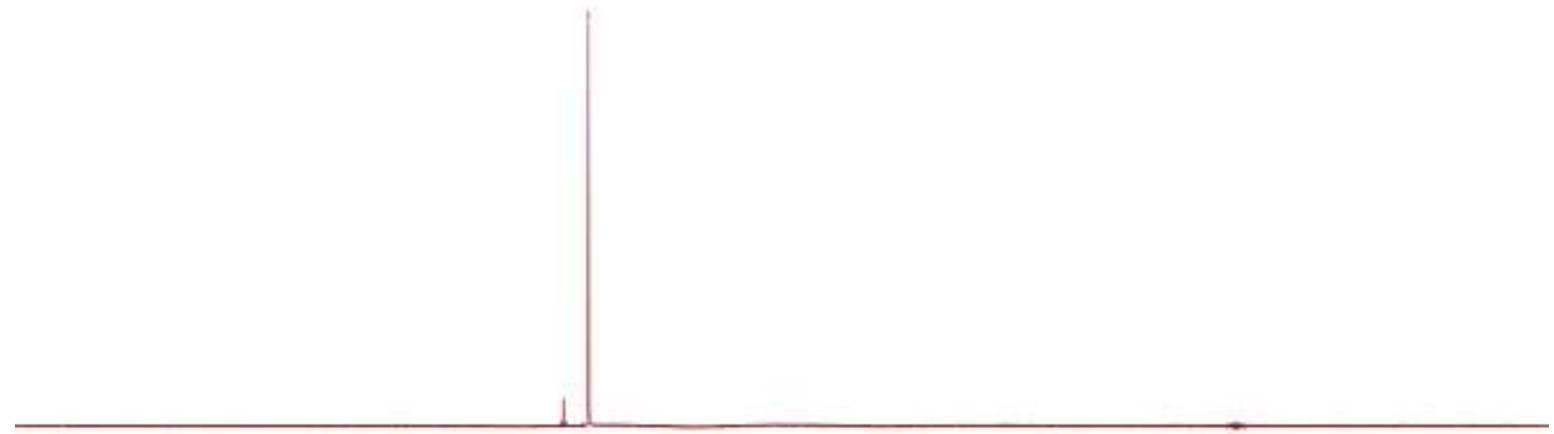

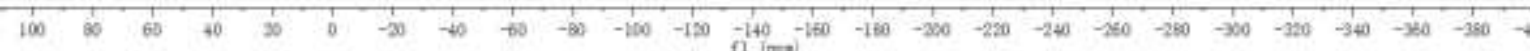




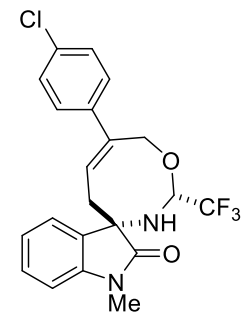

Compound 4ae: Yield: $71 \mathrm{mg}, 84 \%$; A White solid; Mp: 195-197 ${ }^{\circ} \mathrm{C}$; ${ }^{1} \mathrm{H}$ NMR (400 MHz, Chloroform- $d$ ) $\delta 7.45(\mathrm{~d}, J=8.0 \mathrm{~Hz}, 2 \mathrm{H}), 7.41-7.29(\mathrm{~m}, 3 \mathrm{H}), 7.20-7.10(\mathrm{~m}, 1 \mathrm{H}), 7.12-6.99(\mathrm{~m}$, $1 \mathrm{H}), 6.88(\mathrm{~d}, J=8.0 \mathrm{~Hz}, 1 \mathrm{H}), 6.31(\mathrm{t}, J=8.4 \mathrm{~Hz}, 1 \mathrm{H}), 5.73-5.58(\mathrm{~m}, 1 \mathrm{H}), 4.76(\mathrm{~d}, J=13.2 \mathrm{~Hz}, 1 \mathrm{H})$, $4.60(\mathrm{~d}, J=13.2 \mathrm{~Hz}, 1 \mathrm{H}), 3.21(\mathrm{~s}, 3 \mathrm{H}), 2.97(\mathrm{brs}, 1 \mathrm{H}), 2.57$ (brs, $1 \mathrm{H}), 2.46(\mathrm{~d}, J=10.0 \mathrm{~Hz}, 1 \mathrm{H}) ;{ }^{13} \mathrm{C}$ NMR $\left(100 \mathrm{MHz}, \mathrm{CDCl}_{3}\right) \delta 176.9,142.5,139.6,133.5,130.7,129.7,128.7,127.2,127.1,123.0$ (q, $J=279.9 \mathrm{~Hz}), 122.9,122.3,108.8,81.0(\mathrm{q}, J=5.0 \mathrm{~Hz}), 65.6,61.7,35.6,26.2 ;{ }^{19} \mathrm{~F} \mathrm{NMR}(376 \mathrm{MHz}$, Chloroform- $d$ ) $\delta$-80.30; IR (neat): $v$ 3337, 1694, 1616, 1493, 1174, 1153, 1134, 1094, $755 \mathrm{~cm}^{-1}$; HRMS (ESI) Calcd. for $\mathrm{C}_{21} \mathrm{H}_{19} \mathrm{ClF}_{3} \mathrm{~N}_{2} \mathrm{O}_{2}[\mathrm{M}+\mathrm{H}]^{+}$: 423.1082, found: 423.1086.
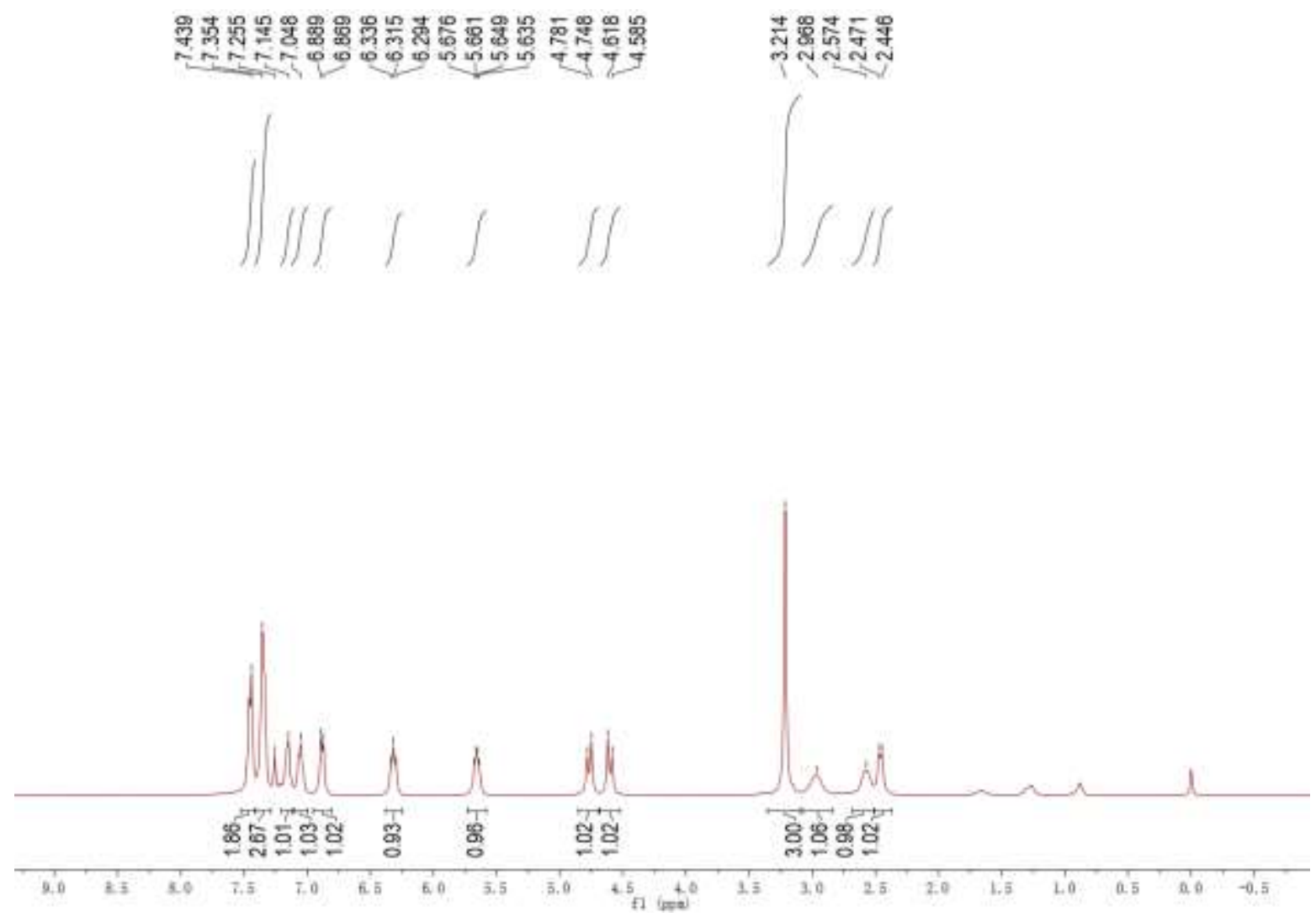


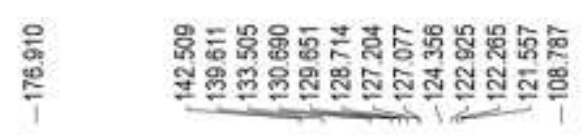

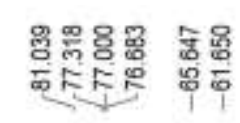

응
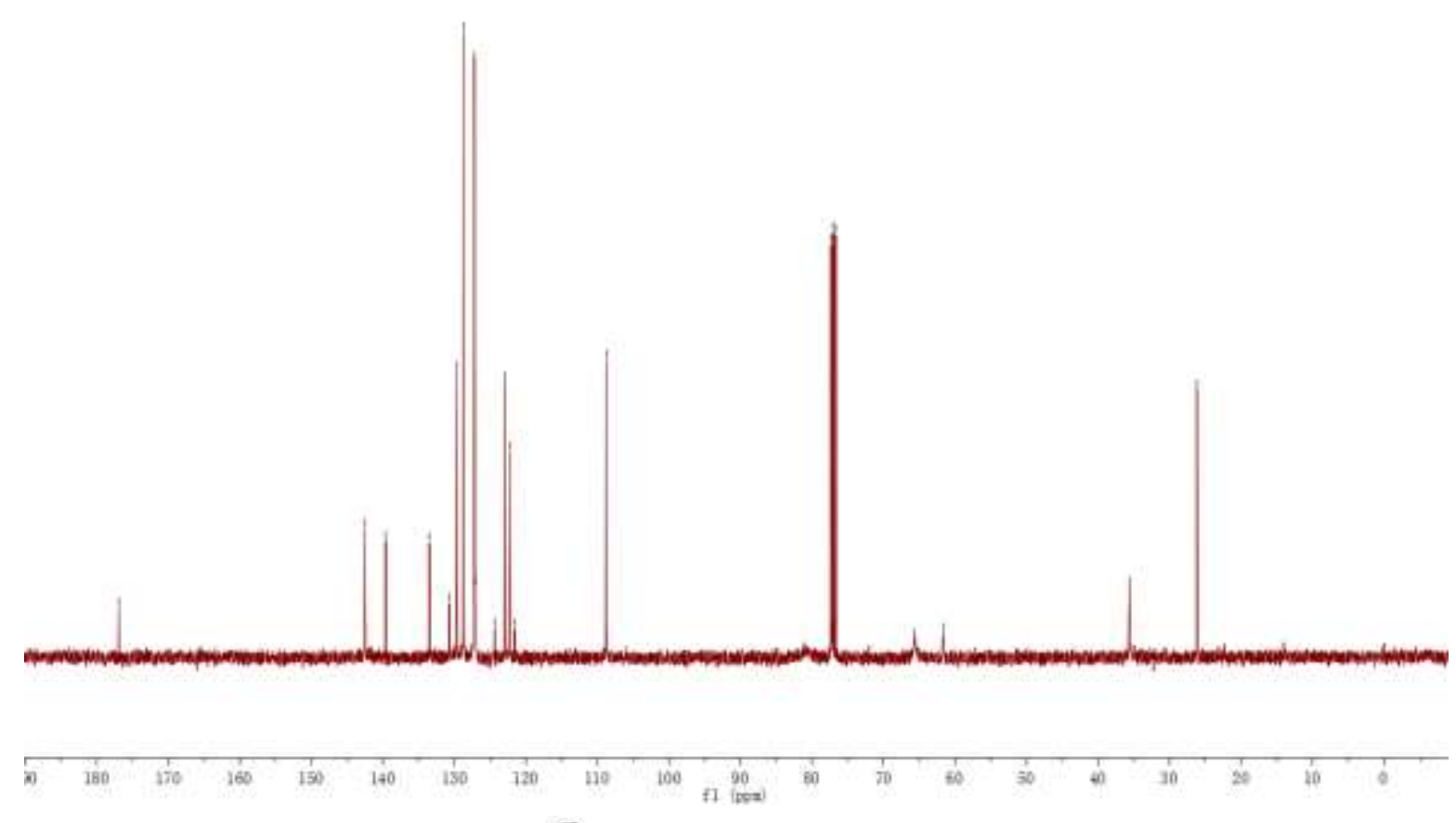

商
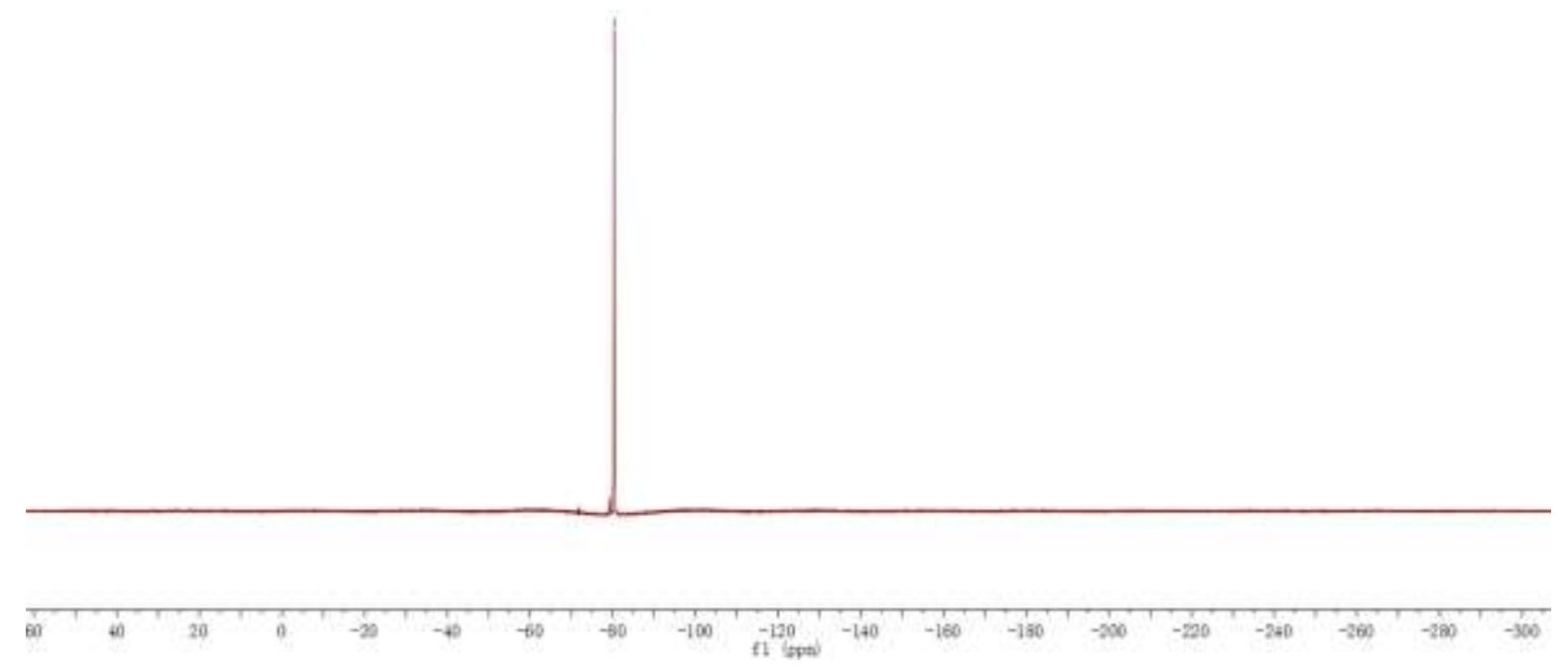

S53 


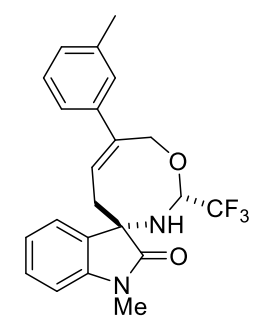

Compound 4af: Yield: $64 \mathrm{mg}, 79 \%$; A White solid; Mp: 160-162 ${ }^{\circ} \mathrm{C}$; ${ }^{1} \mathrm{H}$ NMR (400 MHz, Chloroform- $d$ ) $\delta$ 7.40-7.22 (m, 4H), $7.16(\mathrm{dd}, J=7.6,3.2 \mathrm{~Hz}, 2 \mathrm{H}), 7.03(\mathrm{t}, J=7.6 \mathrm{~Hz}, 1 \mathrm{H}), 6.87(\mathrm{~d}$, $J=7.6 \mathrm{~Hz}, 1 \mathrm{H}), 6.29(\mathrm{t}, J=8.4 \mathrm{~Hz}, 1 \mathrm{H}), 5.74-5.61(\mathrm{~m}, 1 \mathrm{H}), 4.80(\mathrm{~d}, J=13.6 \mathrm{~Hz}, 1 \mathrm{H}), 4.63(\mathrm{~d}, J=$ $13.6 \mathrm{~Hz}, 1 \mathrm{H}), 3.22$ (s, 3H), 3.04 (brs, 1H), 2.66-2.43 (m, 2H), 2.39 (s, 3H); ${ }^{13} \mathrm{C} \mathrm{NMR}(100 \mathrm{MHz}$, $\left.\mathrm{CDCl}_{3}\right) \delta 177.1,142.6,141.2,138.2,130.8,129.6,128.5,128.4,126.6,126.0,123.04,123.02$ (q, $J$ $=279.9 \mathrm{~Hz}), 122.9,122.4,108.7,81.0(\mathrm{q}, J=5.0 \mathrm{~Hz}), 66.3,61.9,35.4,26.2,21.5 ;{ }^{19} \mathrm{~F}$ NMR $(376$ MHz, Chloroform- $d$ ) $\delta$-80.26; IR (neat): v 3343, 2942 1704, 1692, 1179, 1165, 1134, $757 \mathrm{~cm}^{-1}$; HRMS (ESI) Calcd. for $\mathrm{C}_{22} \mathrm{H}_{22} \mathrm{~F}_{3} \mathrm{~N}_{2} \mathrm{O}_{2}[\mathrm{M}+\mathrm{H}]^{+}$: 403.1628, found: 403.1627 .

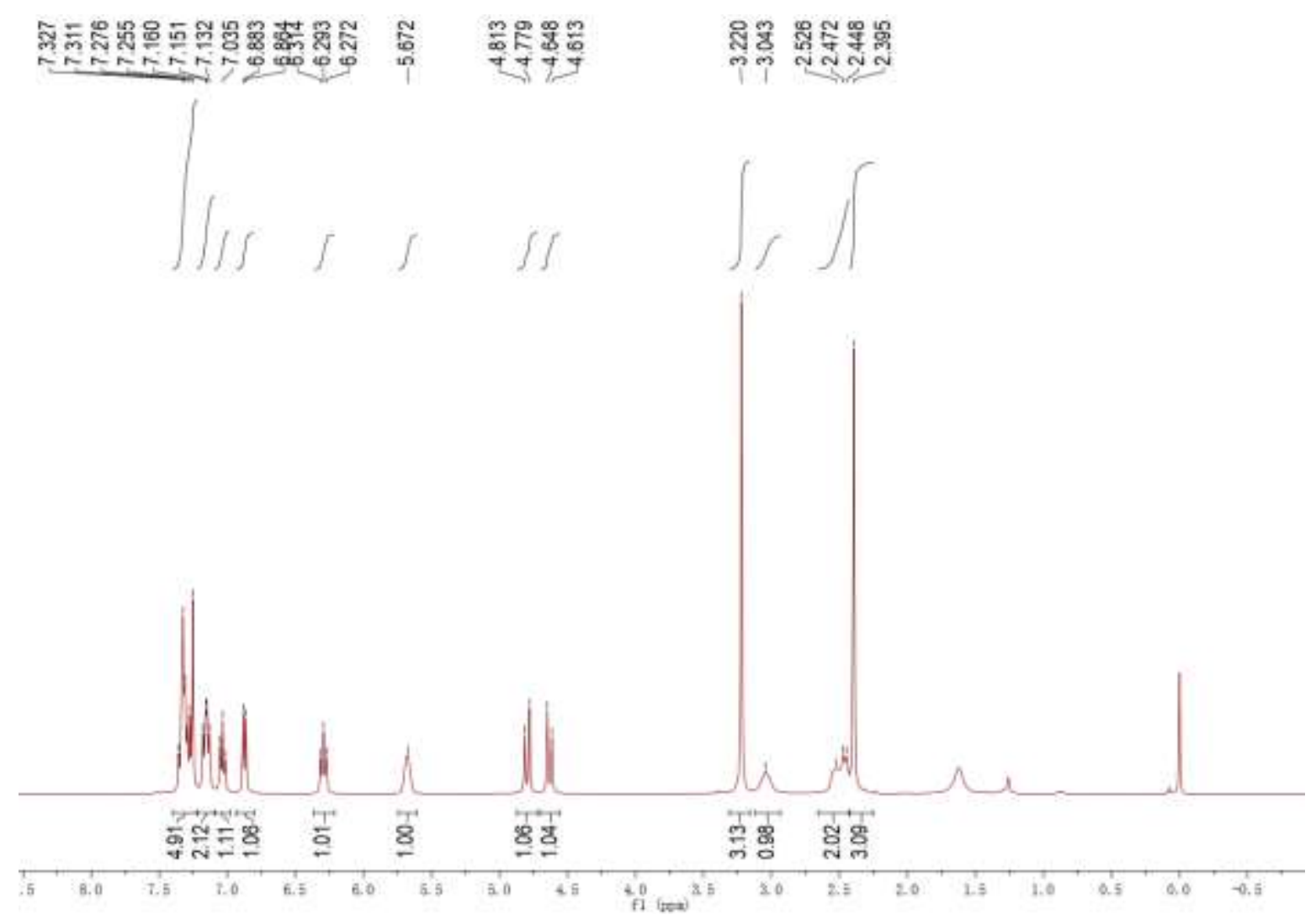



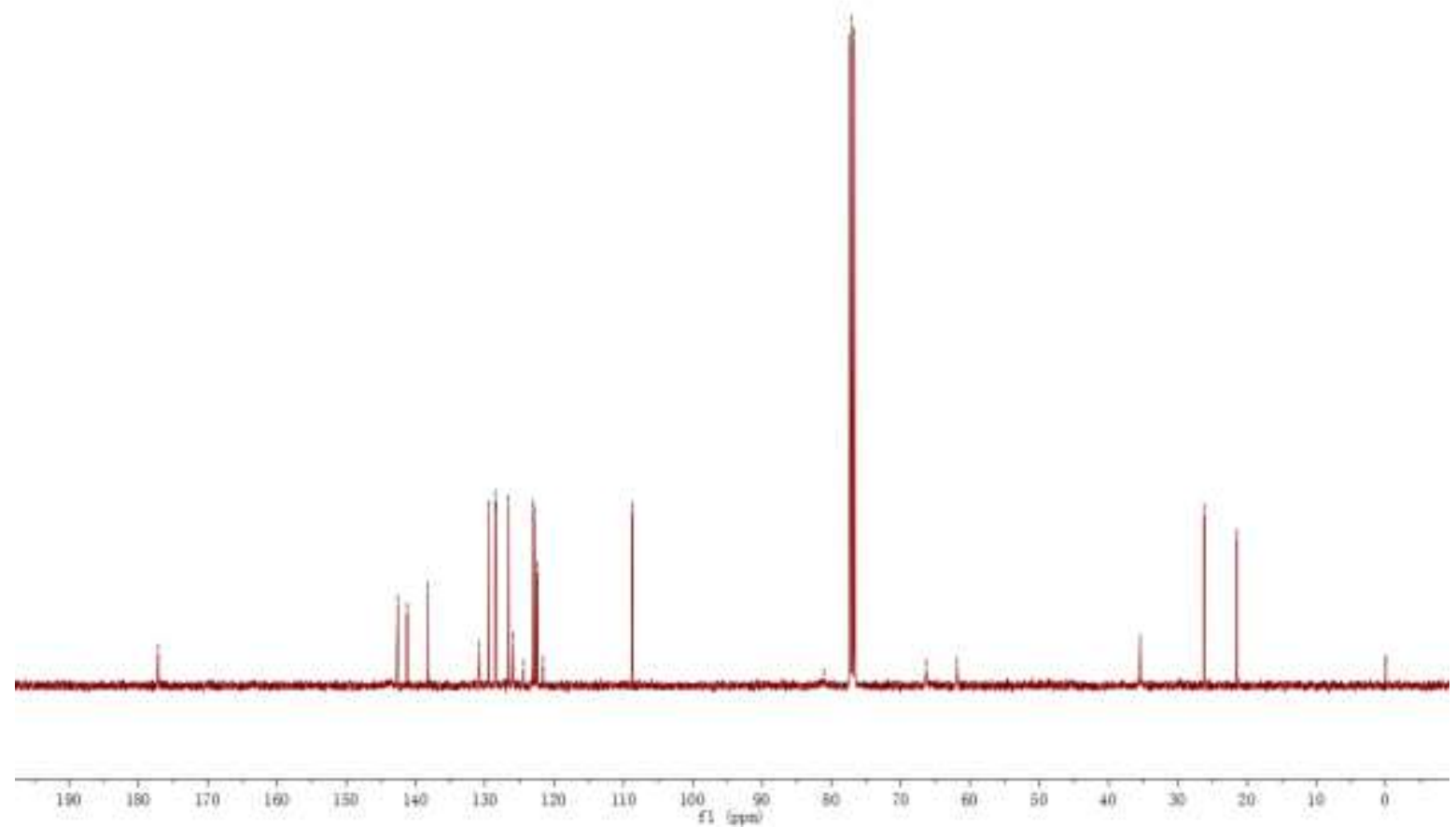

悉

i

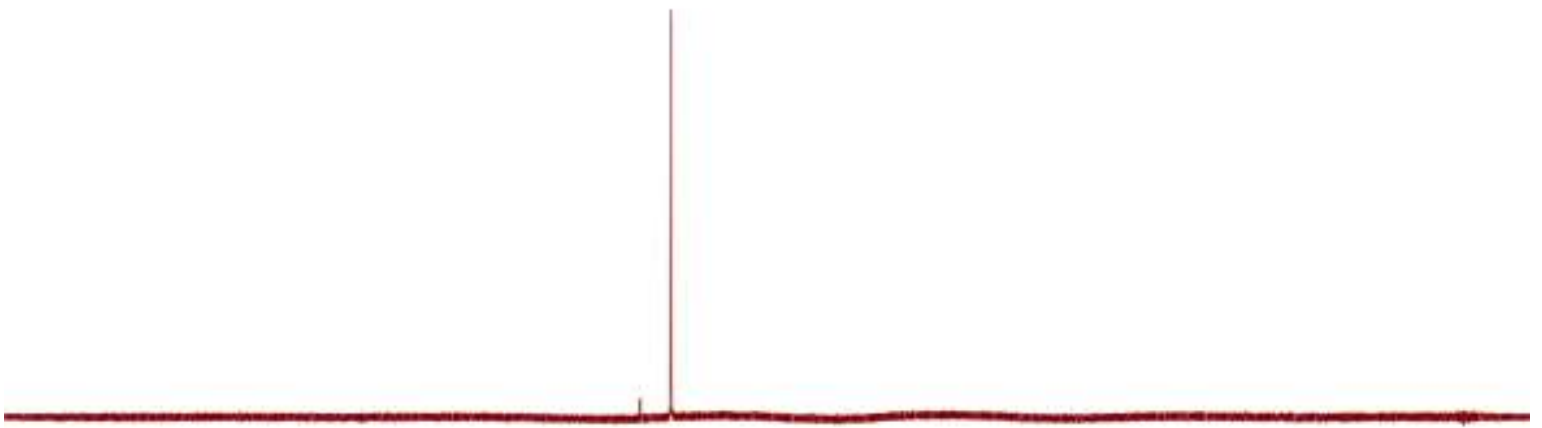

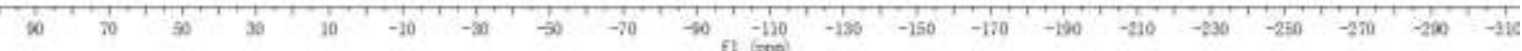




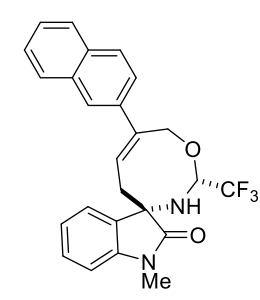

Compound 4ag: Yield: $74 \mathrm{mg}$, 85\%; A White solid; Mp: 171-172 ${ }^{\circ} \mathrm{C}$; ${ }^{1} \mathrm{H}$ NMR (400 MHz, Chloroform- $d$ ) $\delta 7.98(\mathrm{~s}, 1 \mathrm{H}), 7.93-7.75(\mathrm{~m}, 3 \mathrm{H}), 7.75-7.61(\mathrm{~m}, 1 \mathrm{H}), 7.59-7.41(\mathrm{~m}, 2 \mathrm{H}), 7.41-7.28$ (m, 1H), 7.21-7.09 (m, 1H), 7.09-6.95 (m, 1H), $6.87(\mathrm{~d}, J=7.6 \mathrm{~Hz}, 1 \mathrm{H}), 6.47(\mathrm{t}, J=7.6 \mathrm{~Hz}, 1 \mathrm{H})$, 5.84-5.67 (m, 1H), $4.93(\mathrm{~d}, J=13.6 \mathrm{~Hz}, 1 \mathrm{H}), 4.74(\mathrm{~d}, J=13.6 \mathrm{~Hz}, 1 \mathrm{H}), 3.22(\mathrm{~s}, 3 \mathrm{H}), 3.10(\mathrm{~s}, 1 \mathrm{H})$, $2.59(\mathrm{~s}, 1 \mathrm{H}), 2.52(\mathrm{~d}, J=10.0 \mathrm{~Hz}, 1 \mathrm{H}) ;{ }^{13} \mathrm{C} \mathrm{NMR}\left(100 \mathrm{MHz}, \mathrm{CDCl}_{3}\right) \delta 177.1,142.6,138.4,133.4$, $132.8,130.7,129.6,128.3,128.2,127.6,126.9,126.4,126.1,124.6,124.1,123.0$ (q, $J=280.0 \mathrm{~Hz})$, 122.9, 122.4, 108.8, 81.3 (q, $J=5.0 \mathrm{~Hz}), 66.2,61.9,35.6,26.2 ;{ }^{19} \mathrm{~F}$ NMR (376 MHz, Chloroform- $d$ ) $\delta$-80.26; IR (neat): v 3052, 2911, 1708, 1613, 1175, 1089, 818, $747 \mathrm{~cm}^{-1}$; HRMS (ESI) Calcd. for $\mathrm{C}_{25} \mathrm{H}_{22} \mathrm{~F}_{3} \mathrm{~N}_{2} \mathrm{O}_{2}[\mathrm{M}+\mathrm{H}]^{+}:$439.1682, found: 439.1623 .

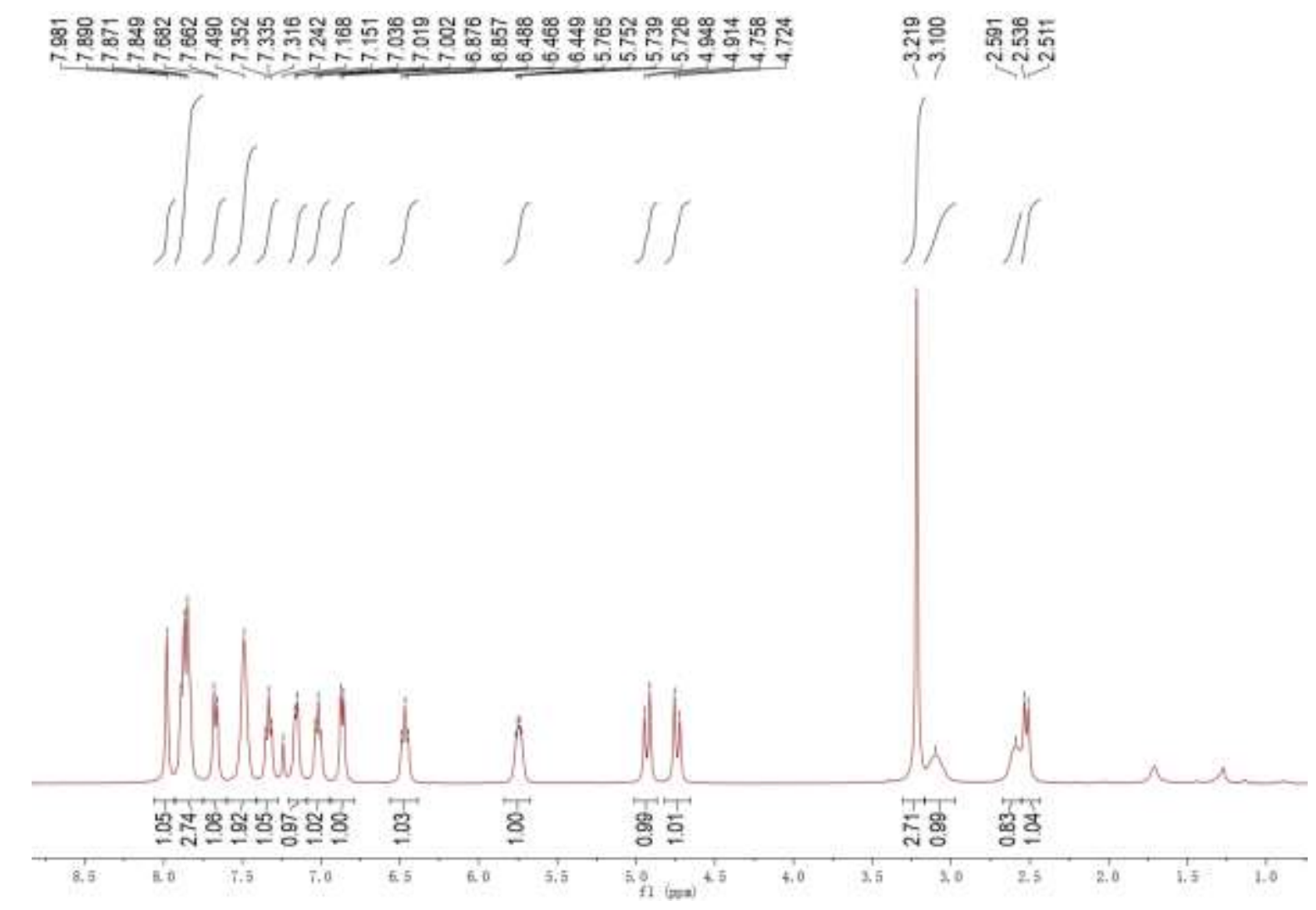



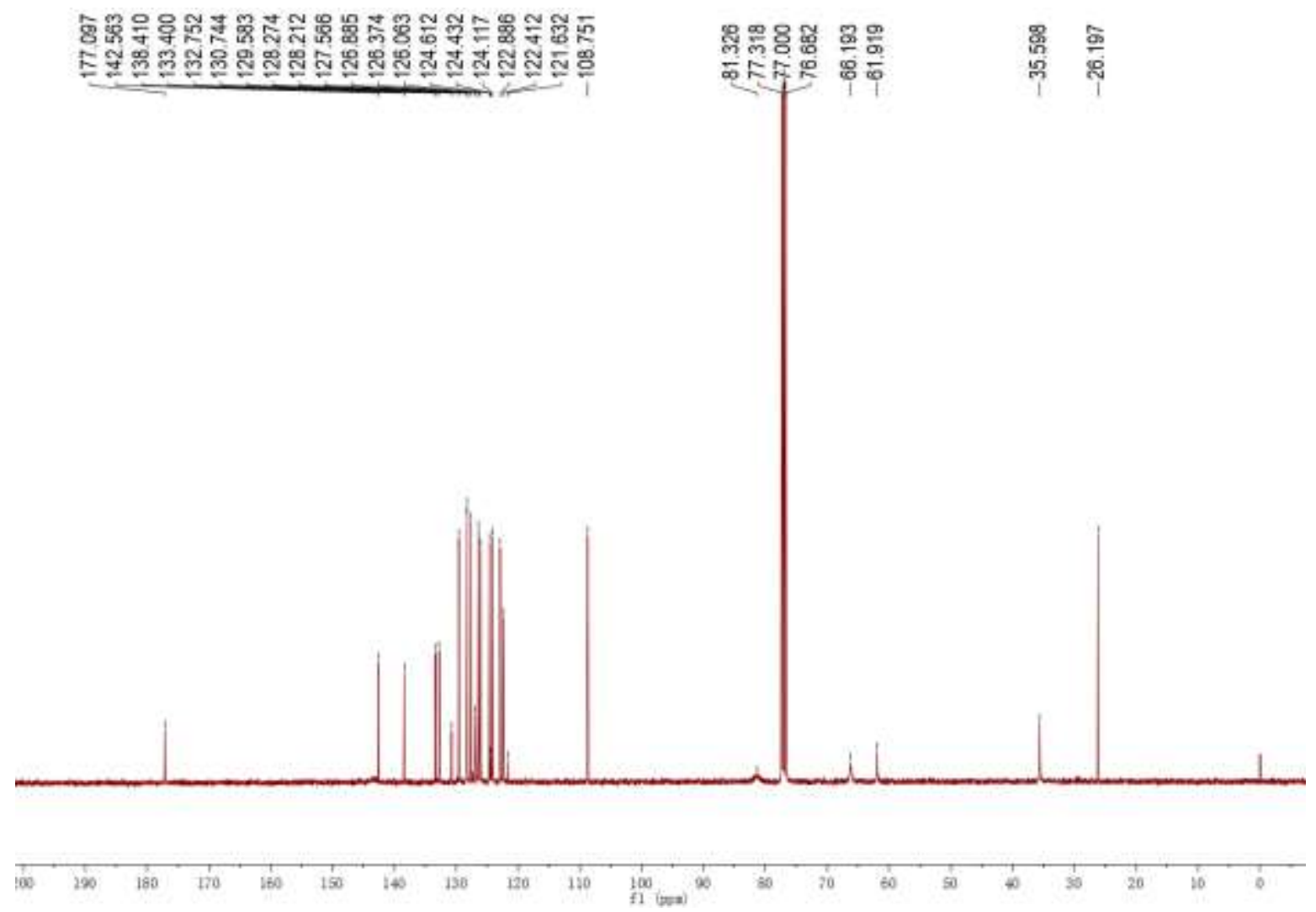

兽

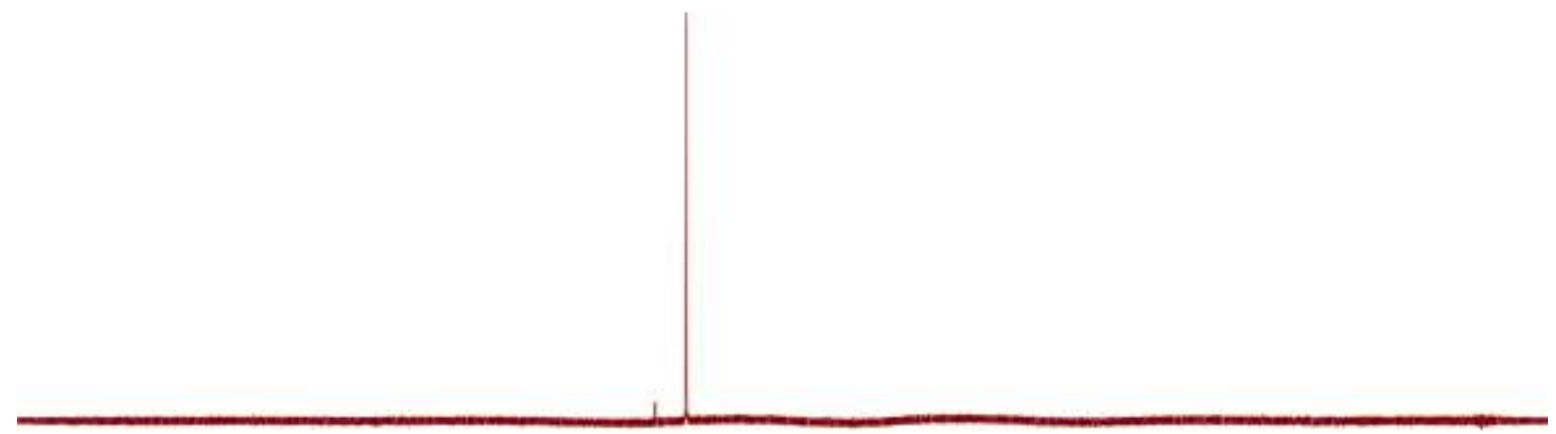

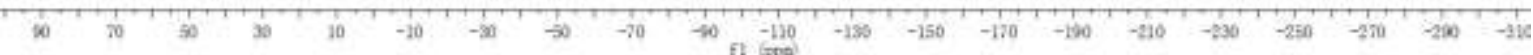




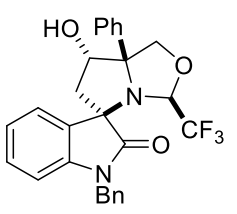

Compound 5a: Yield: $124 \mathrm{mg}$, 63\%; A White solid; Mp: 189-191 ${ }^{\circ} \mathrm{C}$; ${ }^{1} \mathrm{H}$ NMR (400 MHz, Chloroform- $d$ ) $\delta$ 7.68-7.60 (m, 2H), 7.58-7.49 (m, 1H), $7.44(\mathrm{t}, J=8.0 \mathrm{~Hz}, 2 \mathrm{H}), 7.39-7.22(\mathrm{~m}, 7 \mathrm{H})$, $7.18(\mathrm{td}, J=7.6,0.8 \mathrm{~Hz}, 1 \mathrm{H}), 6.82(\mathrm{~d}, J=7.6 \mathrm{~Hz}, 1 \mathrm{H}), 6.16(\mathrm{~d}, J=12.4 \mathrm{~Hz}, 1 \mathrm{H}), 5.12-5.07(\mathrm{~m}, 1 \mathrm{H})$, $5.03(\mathrm{~d}, J=15.6 \mathrm{~Hz}, 1 \mathrm{H}), 4.93(\mathrm{~d}, J=15.6 \mathrm{~Hz}, 1 \mathrm{H}), 4.78(\mathrm{q}, J=6.0 \mathrm{~Hz}, 1 \mathrm{H}), 4.45(\mathrm{dd}, J=12.4,4.4$ $\mathrm{Hz}, 1 \mathrm{H}), 4.00(\mathrm{~d}, J=8.0 \mathrm{~Hz}, 1 \mathrm{H}), 2.54(\mathrm{dd}, J=14.4,4.4 \mathrm{~Hz}, 1 \mathrm{H}), 2.36(\mathrm{~d}, J=14.4 \mathrm{~Hz}, 1 \mathrm{H}) ;{ }^{13} \mathrm{C}$ $\operatorname{NMR}\left(100 \mathrm{MHz}, \mathrm{CDCl}_{3}\right) \delta 178.4,144.8,141.0,134.8,130.9,129.6,129.0,128.5,128.1,127.3$, 127.22, 127.17, 125.7, 124.5, 124.3, 123.1 (q, $J=284.0 \mathrm{~Hz}), 109.9,90.0$ (q, $J=35.0 \mathrm{~Hz}), 84.7$, 80.9, 74.21, 74.20, 44.7, 44.3; ${ }^{19} \mathrm{~F}$ NMR (376 MHz, Chloroform- $d$ ) $\delta$-79.56; IR (neat): v 3345, 16831137 1104, 756, $710 \mathrm{~cm}^{-1}$; HRMS (ESI) Calcd. for $\mathrm{C}_{27} \mathrm{H}_{24} \mathrm{~F}_{3} \mathrm{~N}_{2} \mathrm{O}_{3}[\mathrm{M}+\mathrm{H}]^{+}:$481.1734, found: 481.1722 .

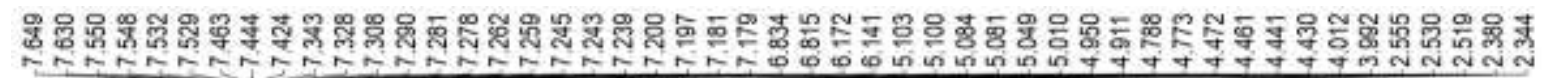

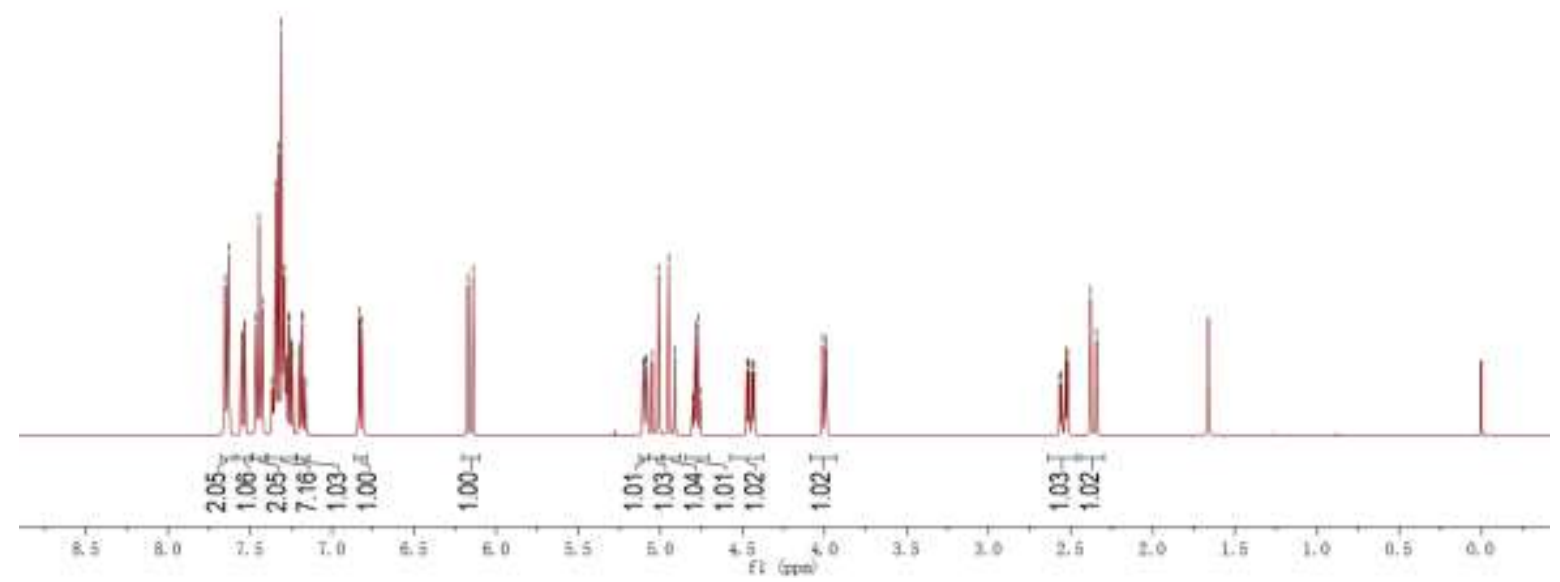




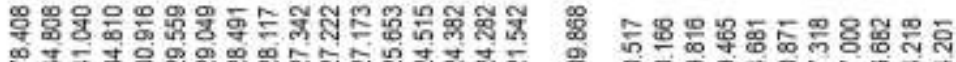

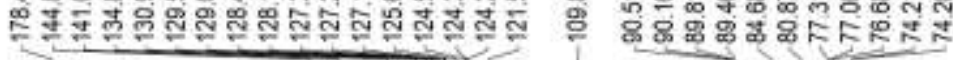

ख 8

+
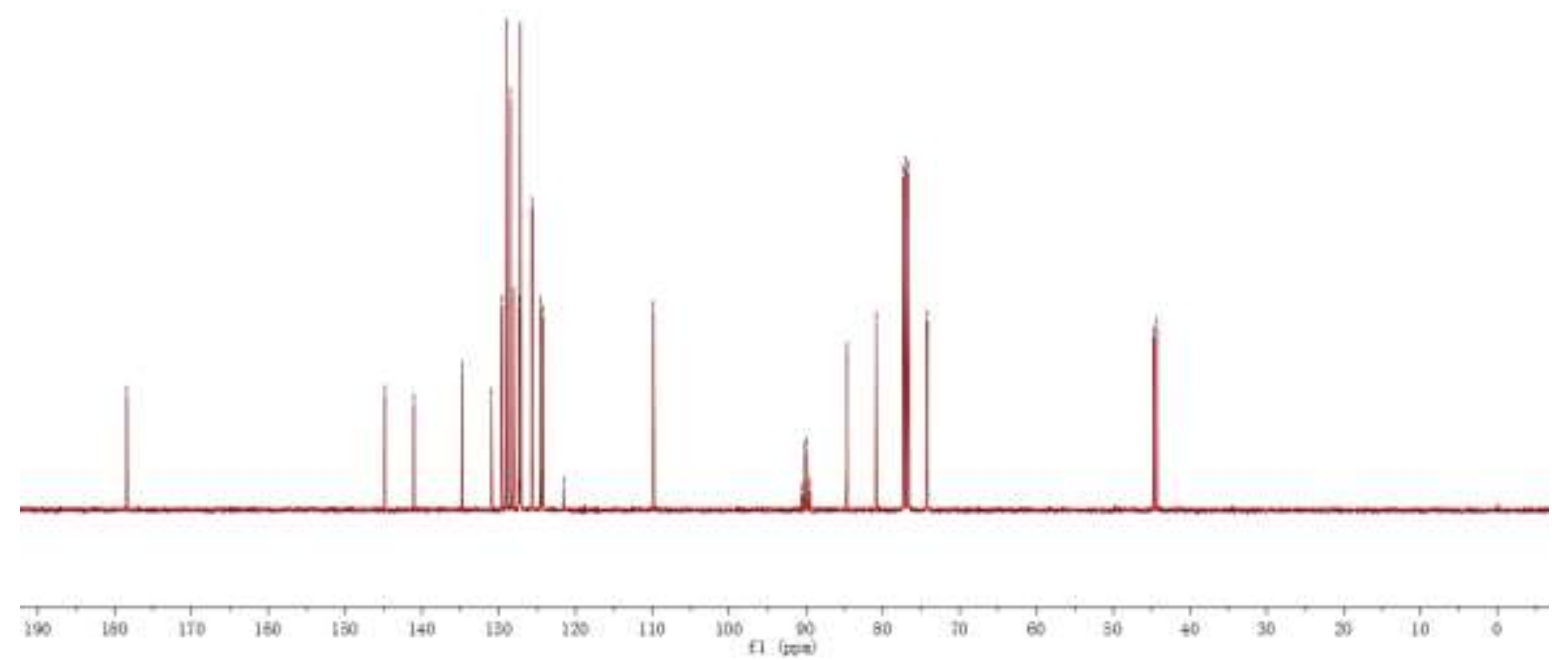

总

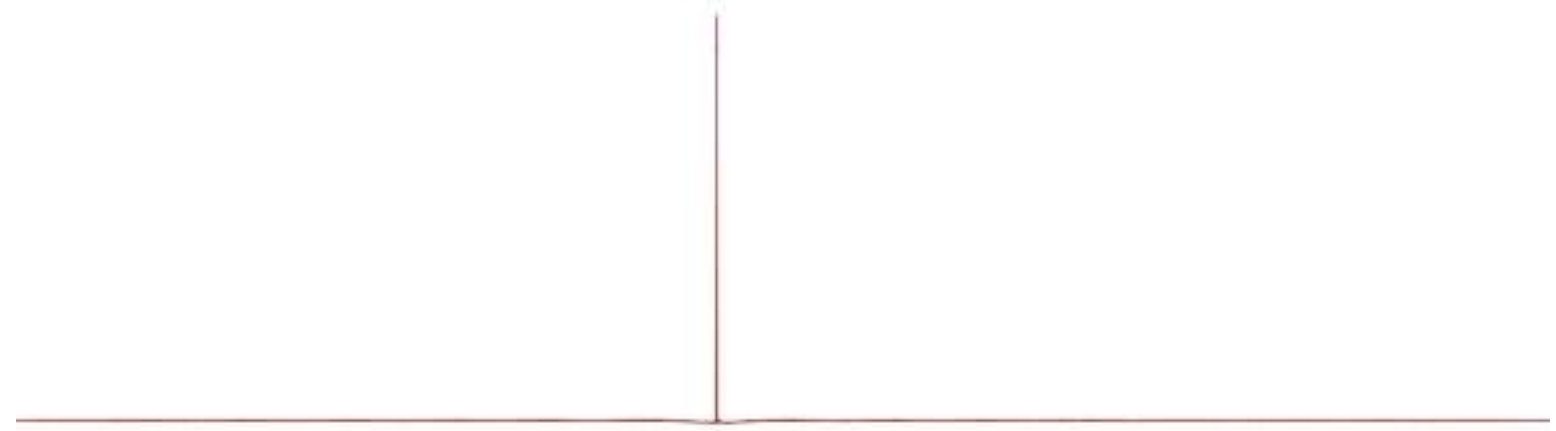

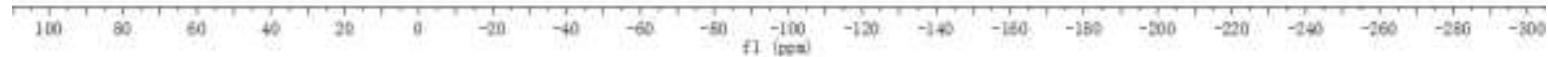




\section{Asymmetric studies}

\subsection{Screening of the chiral ligand}

Table S1: Screening of the chiral ligand when the $N$-protecting group is a benzyl group. ${ }^{\text {a }}$
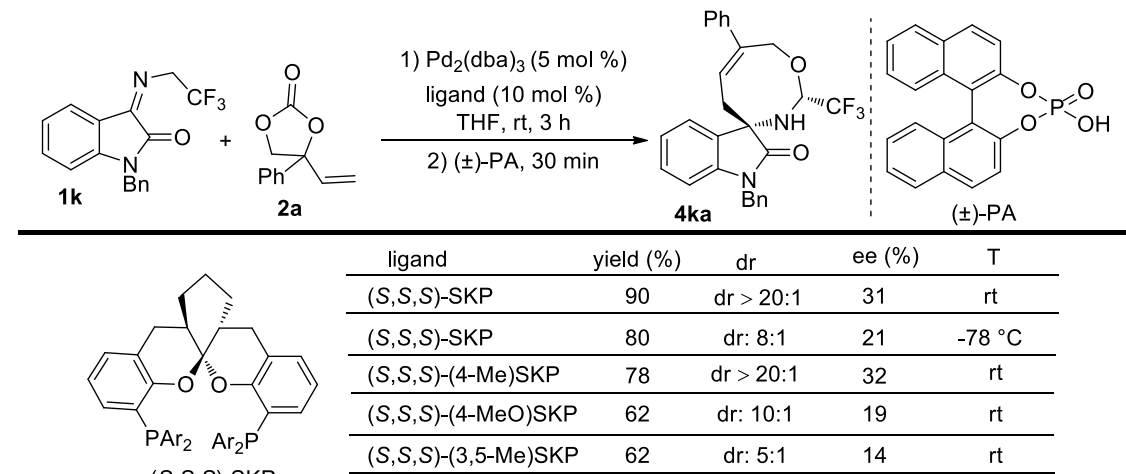

\begin{tabular}{lcccc} 
ligand & yield $(\%)$ & $\mathrm{dr}$ & ee $(\%)$ & $\mathrm{T}$ \\
\hline$(S, S, S)-S K P$ & 90 & $\mathrm{dr}>20: 1$ & 31 & $\mathrm{rt}$ \\
\hline$(S, S, S)-S K P$ & 80 & $\mathrm{dr}: 8: 1$ & 21 & $-78{ }^{\circ} \mathrm{C}$ \\
\hline$(S, S, S)-(4-\mathrm{Me})$ SKP & 78 & $\mathrm{dr}>20: 1$ & 32 & $\mathrm{rt}$ \\
\hline$(S, S, S)-(4-\mathrm{MeO})$ SKP & 62 & $\mathrm{dr}: 10: 1$ & 19 & $\mathrm{rt}$ \\
\hline$(S, S, S)-(3,5-\mathrm{Me}) \mathrm{SKP}$ & 62 & $\mathrm{dr}: 5: 1$ & 14 & $\mathrm{rt}$ \\
\hline$(S, S, S)-(2-\mathrm{Me})$ SKP & 47 & $\mathrm{dr}: 5: 1$ & 11 & $\mathrm{rt}$ \\
\hline
\end{tabular}

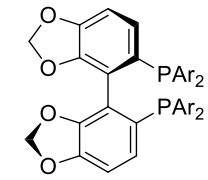

$(R)-(+)$-DM-segphos, trace $(R)-(+)$-DTBM-segphos, complex

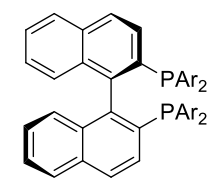

(R)-Binap $\quad 45 \%, \mathrm{~d} r>20: 1,27 \%$ ee (R)-(4-Me)-Binap $41 \%$, dr: 9:1, $27 \%$ ee (R)-Xyl-Binap $24 \%$ dr: $1.5: 1,26 \%$ ee

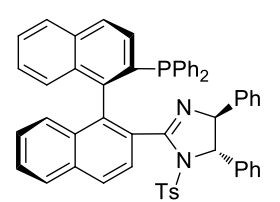

$68 \%, \mathrm{dr}: 6: 1,11 \%$ ee

${ }^{a}$ Reaction conditions: $1 \mathrm{k}(0.26 \mathrm{mmol}), \mathbf{2 a}(0.2 \mathrm{mmol})$, catalyst $(5 \mathrm{~mol} \%)$, ligand $(10 \mathrm{~mol} \%)$ and solvent $(2$ $\mathrm{mL}$ ) were used. ee values were determined by HPLC on a chiral stationary phase.

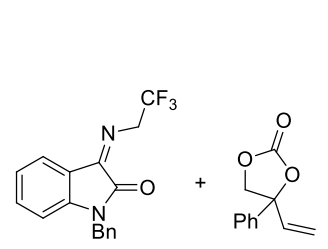

1) $\operatorname{Pd}_{2}(\mathrm{dba})_{3}(5 \mathrm{~mol} \%)$ Xantphos $(10 \mathrm{~mol} \%)$ CPA $(10 \mathrm{~mol} \%)$ DIPEA (100 mol \%) MTBE, rt, $6 \mathrm{~h}$ 2) ( \pm )-PA (20 mol \%) DCM, rt, $15 \mathrm{~min}$

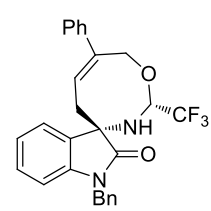

$80 \%$ yield, $d r=1: 1.5,23 \%$ ee<smiles>CCCc1cc(C(C)C)c(-c2cc3ccccc3c3c2O[PH](O)(O)Oc2c(-c4c(C(C)C)cc(C(C)C)cc4C(C)C)cc4ccccc4c2-3)c(C(C)C)c1</smiles>

1) $\mathrm{Pd}_{2}(\mathrm{dba})_{3}(5 \mathrm{~mol} \%)$ Xantphos (10 mol \%) CPA (10 mol \%) DIPEA (100 mol \%) MTBE, rt, $6 \mathrm{~h}$ 2) ( \pm )-PA ( $20 \mathrm{~mol} \%)$
DCM, rt, $15 \mathrm{~min}$

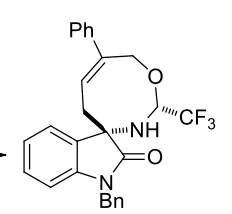

$71 \%$ yield, $d r=1: 6,9 \%$ ee

1) $\mathrm{Pd}_{2}(\mathrm{dba})_{3}(5 \mathrm{~mol} \%)$ Xantphos $(10 \mathrm{~mol} \%)$ CPA $(10 \mathrm{~mol} \%)$ DIPEA (100 $\mathrm{mol} \%)$ MTBE, $\mathrm{rt}, 6 \mathrm{~h}$

2) ( \pm )-PA (20 mol \%) DCM, rt, $15 \mathrm{~min}$

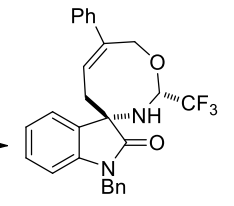

$75 \%$ yield, $d r=1: 5,0 \%$ ee<smiles>C=CC1(c2ccccc2)COC(=O)O1</smiles><smiles>O=P(O)(Oc1c(-c2cc(C(F)(F)F)cc(C(F)(F)F)c2)cc2ccccc2c1-c1cc(C(F)(F)F)cc(C(F)(F)F)c1)Oc1cc(-c2cc(C(F)(F)F)cc(C(F)(F)F)c2)cc2ccccc12</smiles><smiles>O=P(O)(O)Oc1cccc2c1[C@@]13CC[C@@H]2CCc2cccc1c23</smiles>
} 
Table S2: Screening of the chiral ligand when the $N$-protecting group is a methyl group. ${ }^{\text {a }}$
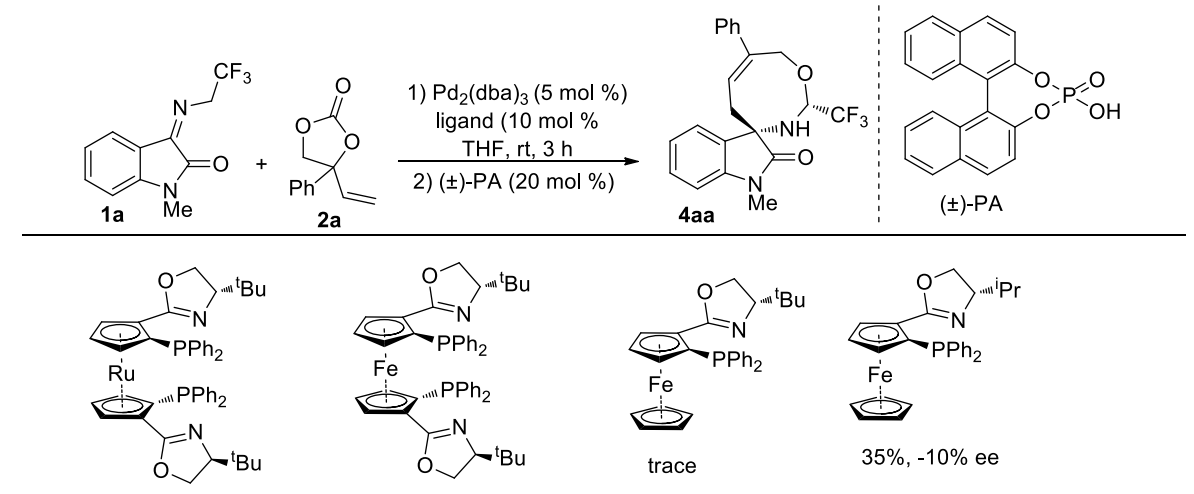

$80 \%, 63 \%$ ee

$81 \%, 35 \%$ ee
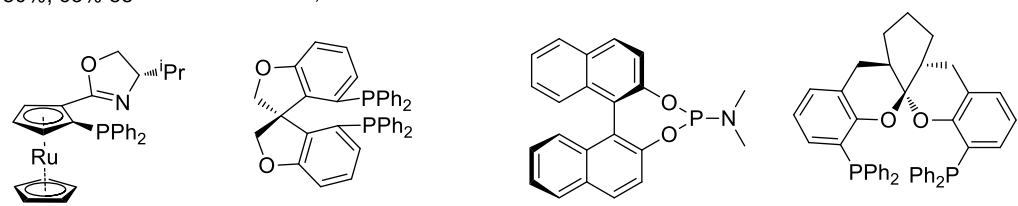

$43 \%, 0 \%$ ee

$71 \%, 0 \%$ ee

Trace

$81 \%, 34 \%$ ee

1) $\mathrm{Pd}_{2}(\mathrm{dba})_{3}(5 \mathrm{~mol} \%)$ L (10 mol \%.) THF, rt, $6 \mathrm{~h}$ 2) (S)-CPA (20 mol \%)
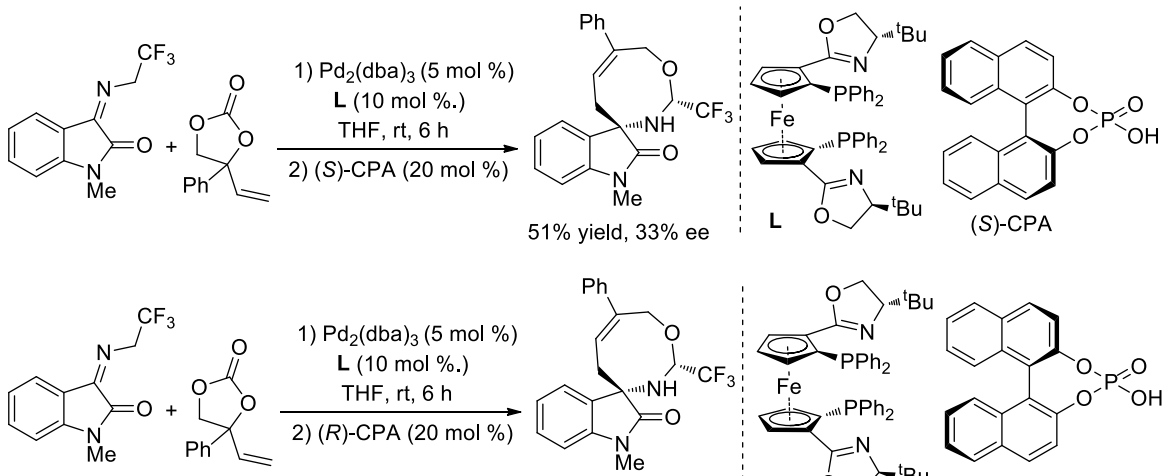

$71 \%$ yield, $34 \%$ ee

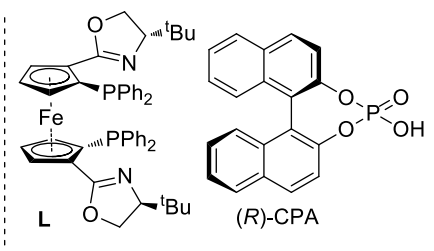

${ }^{a}$ Reaction conditions: $1 \mathrm{a}(0.26 \mathrm{mmol}), \mathbf{2 a}(0.2 \mathrm{mmol})$, catalyst $(5 \mathrm{~mol} \%)$, ligand $(10 \mathrm{~mol} \%)$ and solvent $(2 \mathrm{~mL})$ were used. ee values were determined by HPLC on a chiral stationary phase. 


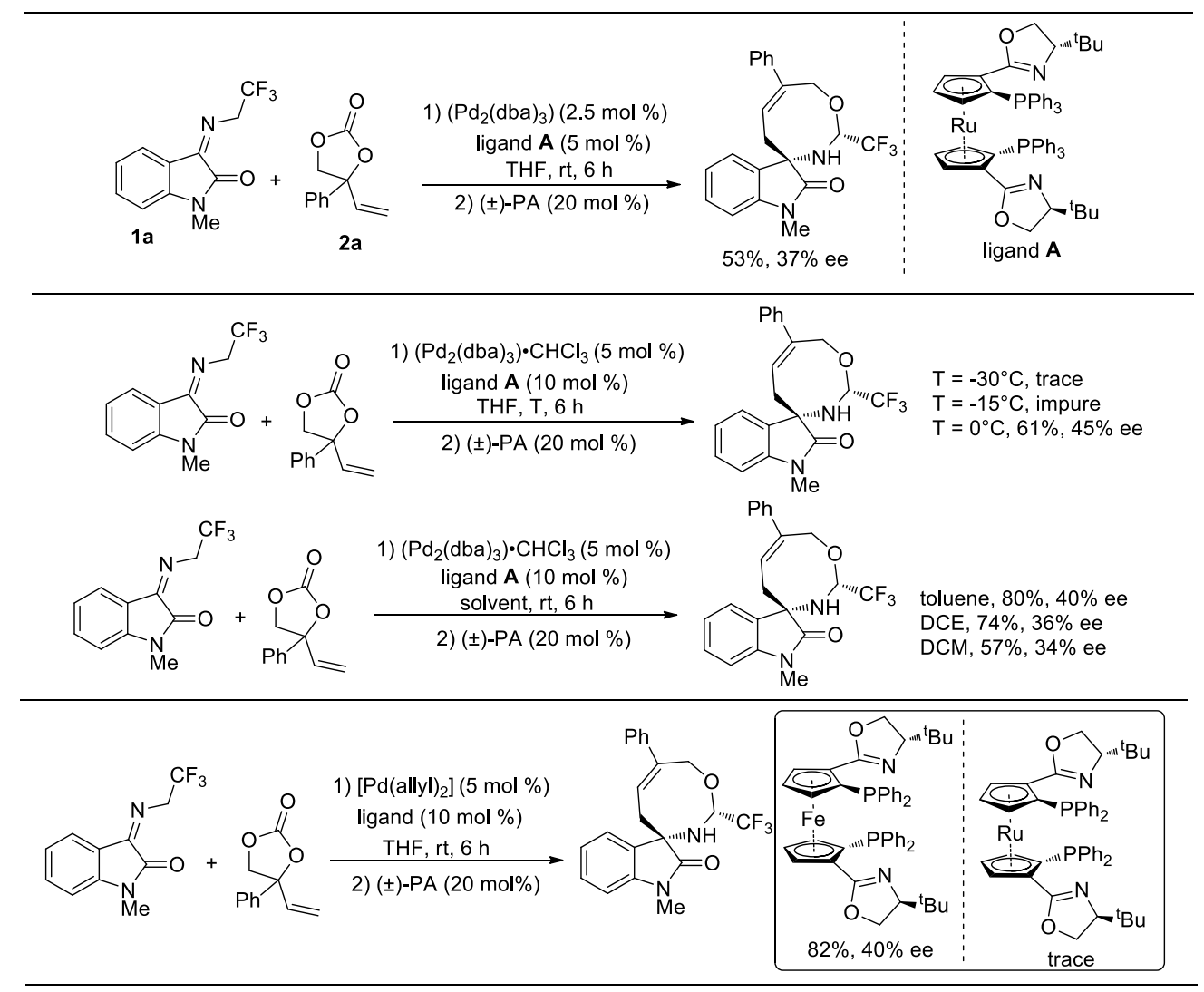

a Reaction conditions: $1 \mathrm{a}(0.26 \mathrm{mmol}), \mathbf{2 a}(0.2 \mathrm{mmol})$, catalyst $(5 \mathrm{~mol} \%)$, ligand $(10 \mathrm{~mol} \%)$ and solvent $(2 \mathrm{~mL})$ were used; ee values were determined by HPLC on a chiral stationary phase.

\subsection{Reaction procedure and HPLC spectra}

In an oven dried $10 \mathrm{~mL}$ Schlenk tube equipped with a magnetic stirring bar, $\left(\mathrm{Pd}_{2} \mathrm{dba}_{3}\right)(0.01$ mmol, 0.05 equiv), and chiral ligand ( $0.02 \mathrm{mmol}, 0.1$ equiv) were added into THF (1.5 mL) under nitrogen atmosphere. The mixture was stirred for $2 \mathrm{~h}$ at room temperature. Then, VEC $\mathbf{2 a}(0.2$ mmol, 1.0 equiv), isatin-derived imide 1a was added and the mixture was stirred for another $2 \mathrm{~h}$. The reaction mixture was stirred at room temperature till almost full conversion of the substrate $\mathbf{2 a}$ by TLC analysis. Then, PA (0.04 mmol, 0,2 equiv) was added and the reaction mixture was continuously stirred for 30 minutes. The solvent was evaporated under vacuum and the residue was purified by a column chromatography (eluent: petroleum ether:ethyl acetate $=10: 1$ to $15: 1$ ) to afford the corresponding product $\mathbf{4 a a}$. 
Product (4aa): a Chiralcel IB-91 column; $\lambda=214$ nm; eluent: Hexane/Isopropanol = 98/2; Flow rate: $0.80 \mathrm{~mL} / \mathrm{min} ; \mathrm{t}_{\mathrm{R}}=9.56 \mathrm{~min}\left(\right.$ minor), $\mathrm{t}_{\mathrm{R}}=10.14 \mathrm{~min}$ (major), enantiomeric ratio: 81.61:18.39. $[\alpha]_{\mathrm{D}}^{20}=+15.1\left(\mathrm{c}=0.360, \mathrm{CH}_{2} \mathrm{Cl}_{2}\right), \mathrm{Mp}: 140-142{ }^{\circ} \mathrm{C}$.
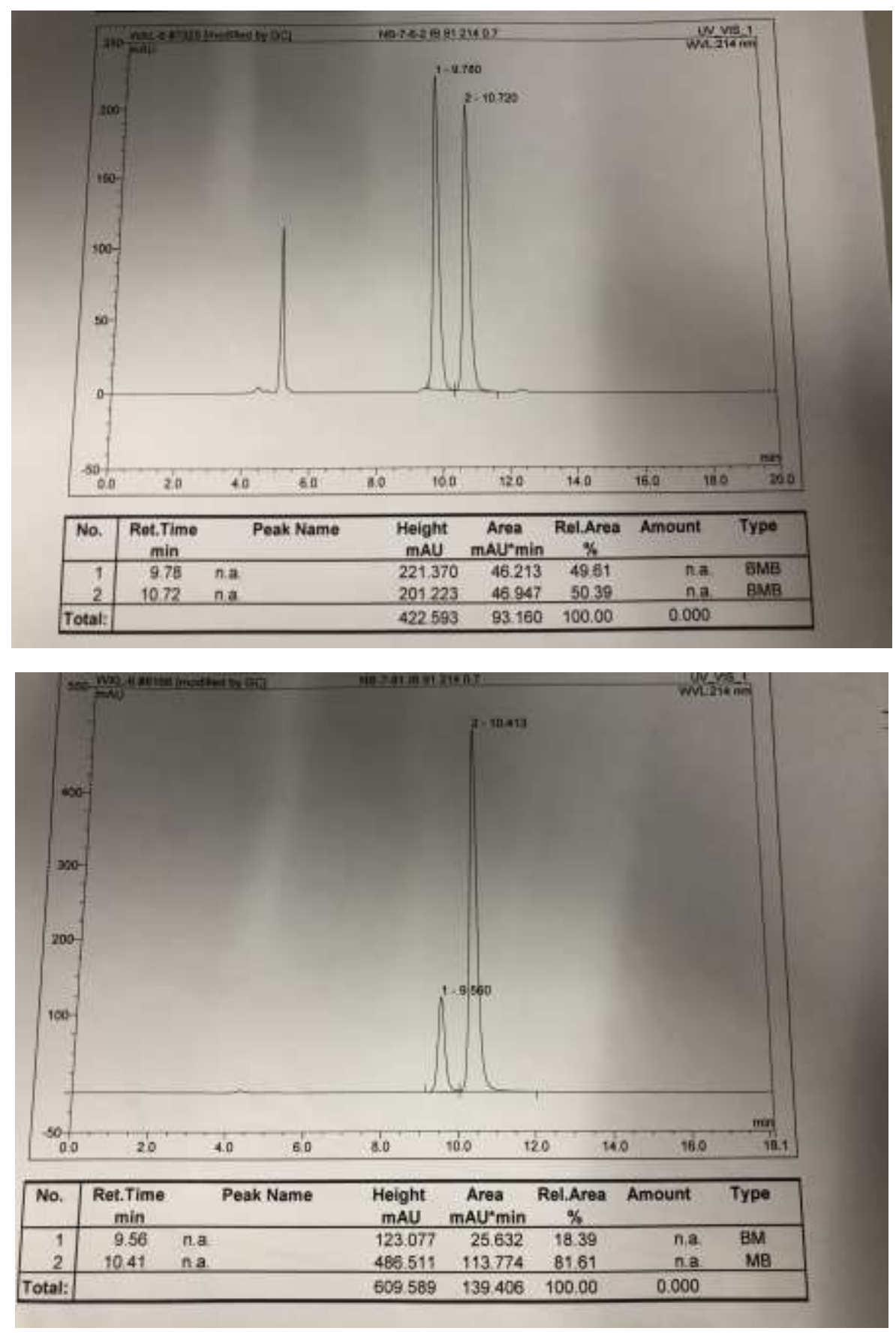


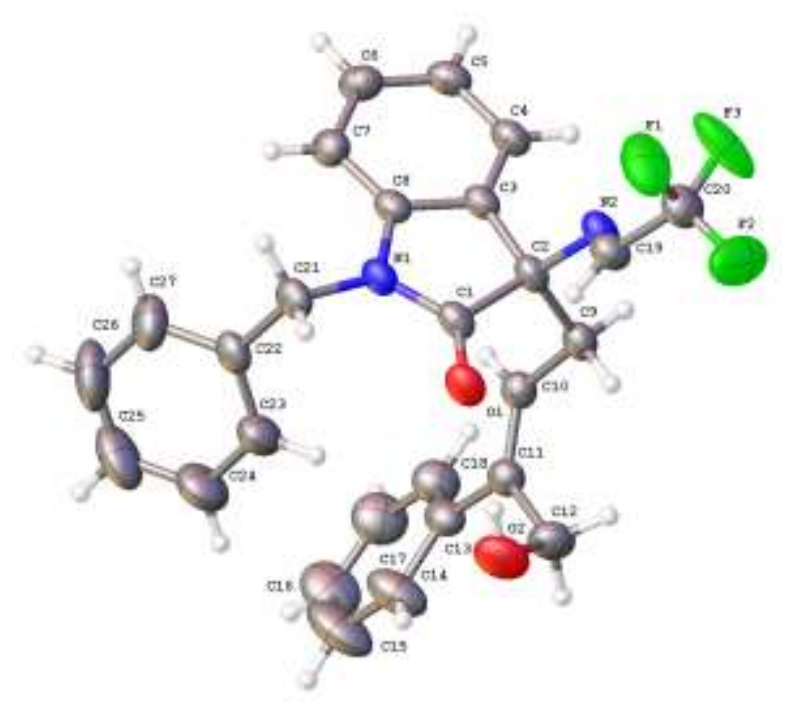

The crystal data of 3ka have been deposited in CCDC with number 1849071. Empirical Formula: $\mathrm{C}_{27} \mathrm{H}_{23} \mathrm{~F}_{3} \mathrm{~N}_{2} \mathrm{O}_{2}$; Formula Weight: 464.47; Crystal Color, Habit: colorless; Crystal Dimensions: 0.15 x 0.1 x $0.05 \mathrm{~mm}^{3}$; Crystal System: Triclinic; Lattice Parameters: $a=9.7709(3) \AA, b=9.8777(3) \AA$, c $=13.9940(4) \AA, \alpha=110.589(2)^{\circ}, \beta=108.505(2)^{\circ}, \gamma=92.243(2)^{\mathrm{o}}, \mathrm{V}=1181.56(6) \AA^{3}$; Space group: $\mathrm{P}-1 ; \mathrm{Z}=2 ; \mathrm{D}_{\text {calc }}=1.306 \mathrm{~g} / \mathrm{cm}^{3} ; \mathrm{F}_{000}=484$; Final $\mathrm{R}$ indices $[\mathrm{I}>2 \operatorname{sigma}(\mathrm{I})] \mathrm{R} 1=0.0511, \mathrm{wR} 2=$ 0.1348 . 


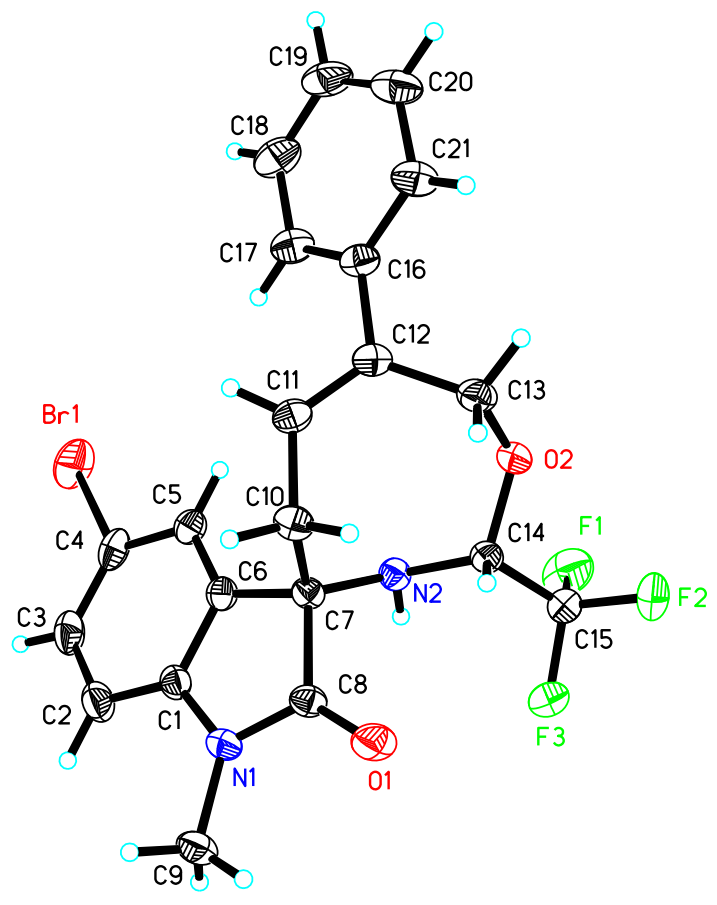

The crystal data of $\mathbf{4 b a}$ have been deposited in CCDC with number 1847596. Empirical Formula: $\mathrm{C}_{21} \mathrm{H}_{18} \mathrm{BrF}_{3} \mathrm{~N}_{2} \mathrm{O}_{2}$; Formula Weight: 467.28; Crystal Color, Habit: colorless; Crystal Dimensions: $0.200 \times 0.180 \times 0.120 \mathrm{~mm}^{3}$; Crystal System: Triclinic; Lattice Parameters: $\mathrm{a}=9.8579(5) \AA, \mathrm{b}=$ $10.5048(4) \AA, c=10.6875(5) \AA, \alpha=116.3120(10)^{\circ}, \beta=90.207(2)^{\circ}, \gamma=93.786(2)^{\mathrm{o}}, \mathrm{V}=989.25(8) \AA^{3}$; Space group: $\mathrm{P}-1 ; \mathrm{Z}=2 ; \mathrm{D}_{\text {calc }}=1.569 \mathrm{~g} / \mathrm{cm}^{3} ; \mathrm{F}_{000}=472 ;$ Final $\mathrm{R}$ indices $[\mathrm{I}>2 \operatorname{sigma}(\mathrm{I})] \mathrm{R} 1=$ $0.0371, \mathrm{wR} 2=0.0944$. 


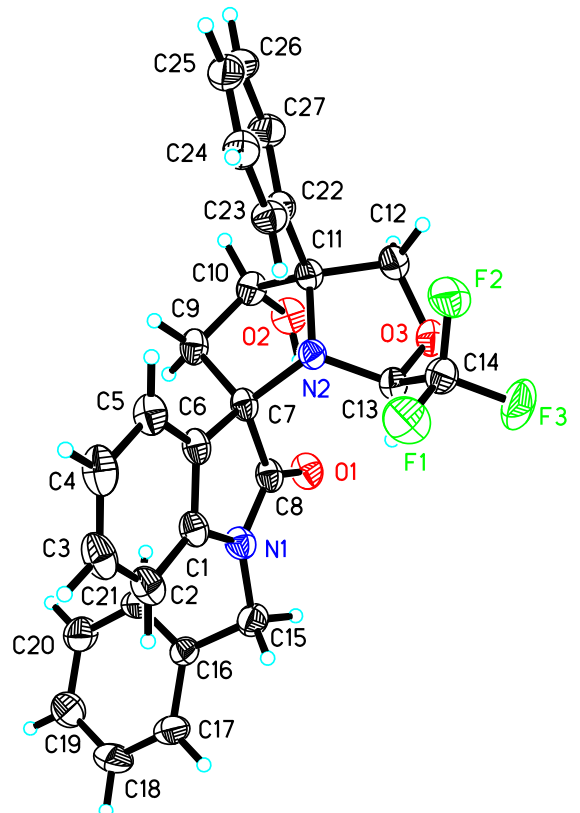

The crystal data of 5a have been deposited in CCDC with number 1853331. Empirical Formula: $\mathrm{C}_{27} \mathrm{H}_{23} \mathrm{~F}_{3} \mathrm{~N}_{2} \mathrm{O}_{3}$; Formula Weight: 480.47; Crystal Color, Habit: colorless; Crystal Dimensions: 0.15 x 0.1 x $0.05 \mathrm{~mm}^{3}$; Crystal System: Monoclinic; Lattice Parameters: a $=8.8419(2) \AA, \mathrm{b}=$

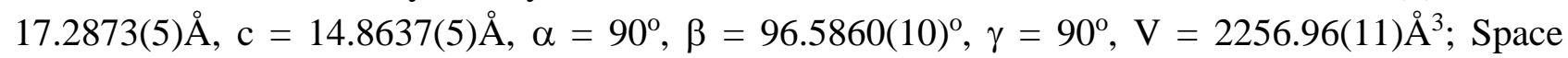
group: $\mathrm{P} 21 / \mathrm{c} ; \mathrm{Z}=4 ; \mathrm{D}_{\text {calc }}=1.414 \mathrm{~g} / \mathrm{cm}^{3} ; \mathrm{F}_{000}=1000$; Final $\mathrm{R}$ indices $[\mathrm{I}>2 \operatorname{sigma}(\mathrm{I})] \mathrm{R} 1=0.0424$, $w R 2=0.1036$. 